\title{
Final Report for DOE Project DE-FC07-99CH11010
}

Project Title: $\quad$ Enhanced Utilization of Corn Based Plastics

Covering Period: September 30, 1999 through June 30, 2003

Date of Report: October 20, 2003

Recipient: $\quad$ Cargill Dow LLC 15305 Minnetonka Blvd Minnetonka, MN 55345

Award Number: DE-FC07-99CH11010

Subcontractors: Professor John R. Dorgan Chemical Engineering Department Colorado School of Mines Golden, CO 80401 USA

Other Partners: $\quad$ Steven Kelley, Ph.D.

NREL

Golden, CO 80401

Contact(s): $\quad$ Jed Randall (952-742-0453) Jed Randall@cargilldow.com

Report Prepared by: Jed Randall \& Robert Kean

Project Team: $\quad$ Amy Manheim (OIT), Matea McCray (ID), Carol VanLente (IDCont) 
Index

1. Abstract

2. Scope changes

3. Measures of progress vs. objectives

4. Final milestone log

5. Intellectual property summary

6. Attachments

7. Publications

8. Key contributors 


\section{Program summary abstract}

Department of Energy award number DE-FC07-99CH11010, Enhanced Utilization of Corn Based Biomaterials, supported a technology development program sponsored by Cargill Dow LLC from September 30, 1999 through June 30, 2003. The work involved fundamental scientific studies on poly lactic acid (PLA), a new environmentally benign plastic material from renewable resources. DOE funds supported academic research at the Colorado School of Mines and the National Renewable Energy Laboratory (NREL), and industry cost share was directed towards applied research into new product development utilizing the fundamental information generated by the academic partners. Under the arrangement of the grant, the fundamental information is published so that other companies can utilize it in evaluating the applicability of PLA in their own products. The overall project objective is to increase the utilization of PLA, a renewable resource based plastic, currently produced from fermented corn sugar.

Three focused areas were pursued to assist in eliminating barriers towards consumer acceptance and wide scale commercialization of PLA. These three breakthrough areas are: Processing and Rheology, Interfacial Engineering, and Molecular Architechture and Phase Morphology.

During the course of the grant, the rheological behavior of PLA was examined in detail. Mathematical models were developed allowing Cargill Dow to predict rheological properties of homopolymers and blends, an important application for ensuring product quality and for maximizing product yield. Model processing studies were conducted to relate rheology to processibility and to elucidate semi-crystalline morphology in PLA fibers. Processing studies relating to thermoforming PLA into a dimensionally stable crystalline articles resulted in significant property improvements, and a patent application. Process studies for stretch injection blow molded bottles were also conducted.

Fundamental issues regarding chain conformations as a function of optical composition were investigated. Structure property relationships relating PLA optical composition to fundamental performance properties such as melting point and rate of crystallization were examined.

Measurements of the permeability of various components through PLA were measured in order to evaluate the suitability of PLA for food and other packaging applications.

Treated films were studied for surface topography and surface chemistry changes. Basic characterization of PLA adhesion to paper substrates was conducted. 


\section{Scope changes}

Naturally, during the course of a research and development project, new information can lead to scope changes. Fortunately, the changes to the scope of this project over the past four years were minimal. Below is a summary of the scope changes that were made.

\subsection{De-emphasis of paper coatings}

Extrusion coating was originally identified as a key focus area, relating to program goals of branching and interfacial adhesion. During the course of the grant, it was realized that development in other areas would have both a faster and larger commercial impact than extrusion coating on to paper substrates, and so a program focus change was made.

The resources at Colorado School of Mines were refocused to fundamentals of solution and melt properties for homopolymers, which required significant synthesis work to make up a well characterized set of model polymers of very wide molecular weight and optical compositions. This was accompanied by a de-emphasis of synthesis of copolymers.

The resources at NREL were redirected to study the surface topography and chemistry of treated films with an emphasis on solving aluminum metallization issues.

\subsection{Addition of surface characterization of PLA films metallized with aluminum}

The focus of this task was broadened to include characterization of PLA surfaces and interfaces with metal (aluminum) coatings.

\subsection{Formation of Clay Composites}

Work in this area was limited due to the demand for resources in other areas. Cargill Dow completed work in this area during the time frame of the grant, using external resources that were not allocated to the grant. Program coordination of clay composites was under the direction of Richard C. Bopp of Cargill Dow.

\section{Measures of progress vs. objectives}

\subsection{Processing and Rheology}

\subsubsection{Measurement of Molecular Sizes and Conformations}

The previous literature for Polylactides (PLAs) on basic chain properties and solution characterization is disjointed and inconsistent. In this project, a comprehensive and well-controlled set of experiments combined with consistent numerical analyses was completed in order to resolve existing apparent contradictions. Homopolymers and copolymers spanning wide ranges of molecular mass and stereoisomer proportions were prepared by ring-opening polymerizations of pure or mixed L- and D-lactide stereoisomeric dimers. The polymer products were characterized by means of: (1) dilute-solution viscometry in three different solvents; (2) size-exclusion chromatography in THF with light-scattering detection; (3) static multiangle light scattering in a mixed acetonitrile-dichloromethane solvent; (4) variable-angle spectroscopic ellipsometry; and 
(5) melt rheology.

\title{
3.1.1.1. Absolute molecular weight determination of PLA optical copolymers by GPC-LLS
}

This work was conducted at the Colorado School of Mines. Molecular weights determined by light scattering in two different solvents generally agreed to within the uncertainty of the measurement. Careful experimental and theoretical determinations of refractive indexes of the polymers and solvents used showed that refractive index increments used in some earlier published light-scattering determinations of PLA molecular weights were incorrect. The molecular weight values (determined via light scattering) were primarily used for the development of the viscosity/molecular weight calibrations and constants described below. The results summarized here were initially reported in the Q2-2002 quarterly report, with further work described in the Q3 and Q4 reports of 2002. A summary of the work is in the following publication submission:

Fundamental Solution and Single-Chain Properties of Polylactides. Dorgan, John R., Janzen, Jay, Knauss, Daniel M., Hait, Sukhendu B., Limoges, Bradford R., and Hutchinson, Matthew $\mathrm{H}$.

\begin{abstract}
Polylactides (PLA) have been known for several decades and have recently become of considerable commercial significance. However, the literature on basic chain properties and solution characterization is divided and inconsistent. In this study, a comprehensive and well-controlled set of experiments is combined with rigorous quantitative analysis in order to resolve existing apparent contradictions. Homopolymers and copolymers spanning wide ranges of molecular weight and stereoisomer proportions were prepared by ring-opening polymerizations of L- and D-lactides using stannous octanoate as catalyst. Samples were characterized by means of: 1) dilute-solution viscometry in three different solvents; 2) size-exclusion chromatography in THF with light-scattering detection; 3) static multiangle light scattering in a mixed acetonitrile-dichloromethane solvent; 4) variable-angle spectroscopic ellipsometry; and 5) melt rheology. The data imply polylactides are typical linear flexible polymers; unperturbed PLA chain dimensions as describable in terms of a characteristic ratio of $6.5 \pm 0.9$, regardless of stereoisomer content. The Schulz-Blaschke and Mark-Houwink constants for dilute PLA solutions in chloroform and in THF are determined. For chloroform at $30^{\circ} \mathrm{C}$ the correct values are $k_{S B}=0.302, K=0.0131(\mathrm{~mL} / \mathrm{g})$ and $a=0.759$ while for THF at $30{ }^{\circ} \mathrm{C}$ the correct values are $k_{S B}=0.289, K=0.0174(\mathrm{~mL} / \mathrm{g})$ and $a=0.736$. Experimental evidence to prefer unperturbed chain-dimension (theta-state) estimates based on Stockmayer-Fixman analysis over Orofino-Flory analysis of second virial coefficients is presented.
\end{abstract}

\subsubsection{Dilute solution properties of PLA optical copolymers in a variety of solvents}

Experimental details and results for this task were reported in the Q4-2002 progress report, and support the results presented in the subsections below.

Unperturbed PLA chain dimensions are describable in terms of a characteristic ratio in the range $6.5 \pm 0.9$, regardless of stereoisomeric composition. This implies that polylactides are typical linear flexible polymers.

Precise Mark-Houwink and Schulz-Blaschke constants for dilute PLA solutions in 
chloroform and in THF were determined: For chloroform at $30^{\circ} \mathrm{C}, K=0.0131 \mathrm{~mL} / \mathrm{g}, a=$ 0.777 , and $k_{\mathrm{SB}}=0.302$; for THF, $K=0.0174 \mathrm{~mL} / \mathrm{g}, a=0.736$, and $k_{\mathrm{SB}}=0.289$. These results and supporting data are reported in the publication submission above.

\title{
3.1.1.3. Mathematical model for single point relative viscosity characterization tests to yield absolute weight averaged molecular weight.
}

Having a well-determined Schulz-Blaschke coefficient for PLA's in chloroform in addition to Mark-Houwink constants also implies feasibility of making accurate determinations of molecular weights via one-point dilute-solution relative viscosity measurements. The ideal concentration for such determinations from a single relative viscosity test is 0.3 $\mathrm{g} / \mathrm{dL}$ for an approximate molecular weight range from 100,000 to 300,000 . A preliminary model is functional based on the coefficients reported above and in previous progress reports. Cargill Dow, following completion of the project, will refine additional refinements to the model coefficients.

\subsubsection{Molecular weight determination by dielectric spectroscopy}

A summary of the work is in the following publication.

Local and global dynamics of polylactides. A dielectric spectroscopy study. Mierzwa, M.; Floudas, G.; Dorgan, J.; Knauss, D.; Wegner, J. IESL, Foundation for Research and Technology-Hellas (FORTH), Heraklion Crete, Greece. Journal of Non-Crystalline Solids (2002), v 307-310, 296-303.

\begin{abstract}
Polylactides are a family of degradable plastics having a component of the dipole moment both perpendicular and parallel to the polymer backbone (i.e. is a type-A polymer). We have studied the sub-glass, segmental and global chain dynamics in a series of fully amorphous samples having an L:D ratio of $80: 20$, with mol. wts. in the range $3.8 \times 10^{3}<M_{n}<4.7 \times 10^{4}$. Finite size effects have been obsd. for both the segmental and the sub-glass process. The segmental mode was found to have a steeper temp. and pressure dependence compared to the longest normal mode in accord with findings from other type-A polymers. The mol. wt. dependence of the longest normal mode relaxation times ( $\tau$.apprx. M3.1n) reflects on the intermediate mol. wt. regime $\left(\mathrm{M}_{\mathrm{e}}\right.$.apprx. $\left.6 \times 10^{3}\right)$ from Rouse to entangled chains. These findings are compared with the viscoelastic results on the same system. Agreement in the $\mathrm{C}_{2}$ value of the WLF equation is found but the scaling of the normalized longest relaxation time derived from the rheol. measurements shows a stronger than expected dependence on the mol. wt.
\end{abstract}

\subsubsection{Preparation and Characterization of PLA Blends}

\subsubsection{Blends of linear PLAs of different molecular weights}

The rheology of linear PLA blends was investigated, and is summarized in the section below.

\subsubsection{Blends of linear and branched PLAs}

Blends of linear and branched materials can offer processing advantages, specifically in terms of tailored melt strength and ability to flow in shear or extension. Most of the work conducted in this and the previous subtask has been published in the journal article 
listed below. The abstract of the paper follows.

Melt Rheology of Poly(lactic acid): Consequences of Blending Chain Architectures - H.J. Lehermeier, and J.R. Dorgan Polymer Engineering and Science, 2001, 41, No. 12, 2172-2184.

\begin{abstract}
The rheology of blends of linear and branched poly(lactic acid) (PLA) architectures is comprehensively investigated. Measurement of the melt rheological properties of PLA is complicated by degradation effects but the addition of $0.35 \mathrm{wt} . \%$ tris(nonylphenyl) phosphite (TNPP) provides excellent stabilization over a range of temperatures. Master curves of dynamic viscosity constructed using time temperature superposition show significant dispersion for unstabilized samples; this behavior is accompanied by a loss of molecular weight. TNPP stabilized samples show excellent superposition throughout the entire frequency range and minimal loss in molecular weight. For the linear architecture, the Cox-Merz rule is valid for a large range of shear rates and frequencies. The branched architecture deviates from the Cox-Merz equality and blends show intermediate behavior. Both the zero shear viscosity and the elasticity (as measured by the recoverable shear compliance) increase with increasing branched content. The viscosities of both the unstabilized samples and the TNPP stabilized samples roughly obey a log additivity mixing rule. The recoverable shear compliance is monotonic in blend composition and a mixing rule for this property is also presented. For the linear chain, the compliance is independent of temperature but this behavior is apparently lost for the branched and blended materials. Tensile and thermal properties of the blends are also measured and found to be roughly equal within the statistical error of the experiments. The results suggest that excellent control over rheological behavior of PLA is possible through blending chain architectures without compromising mechanical properties.
\end{abstract}

\title{
3.1.2.3. Blends of PLAs of different optical compositions
}

Studies of melt rheology on a series of PLA samples with different ratios of L:D (50:50, $80: 20,100: 0)$, showed no significant dependence of viscosity on L:D ration. These results were summarized in the Q2, 2002 progress report. In addition, a recent publication submission captures the effects of different $L: D$ ratios on subsequent flow behaviors.

Melt Rheology of Variable L-content Poly(lactic acid). Janzen, Jay, Dorgan, John R., Clayton, Michael P., Knauss, Daniel M., and Hait, Sukhendu B. submitted to Macromolecules, 2003.

\footnotetext{
Abstract

Polylactides (PLAs) have been known for several decades and have recently gained considerable commercial significance. This development makes it urgently desirable to have the rheological properties of these materials well-characterized. However, comprehensive rheological characterization of PLAs is not yet available from the literature. In this study, rheological and thermal measurements were made on a comprehensive and well-characterized set of homopolymers and copolymers spanning wide ranges of molecular mass and stereoisomer proportions (L-content). For the weight average molecular weight range of $10^{5}$ to $10^{6}(\mathrm{~g} / \mathrm{mol})$ and a reference temperature of $180 \mathrm{C}$, the zero shear viscosity is described well by the relationship $\log \left(\eta_{0}\right)=-14.26+3.4 \log \left(M_{w}\right)$ regardless of the stereochemical composition. Glass
} 
transition temperatures do show a trend of increased values with increased L-content; the values of the glass transition temperatures at infinite molecular weight for 100, 80, and $50 \%$ L-content are $60.2^{\circ} \mathrm{C}, 56.4^{\circ} \mathrm{C}$, and $54.6^{\circ} \mathrm{C}$, respectively. The data imply polylactides are typical linear glass-forming polymers. Unperturbed PLA chain dimensions well above their glass transition temperatures are describable in terms of a characteristic ratio in the range $6.5 \pm 0.9$, independent of stereoisomeric composition.

In addition, blends of varied optical compositions were examined to investigate processing and property improvements, specifically with regard to crystallization and stretching behavior. The studies utilize and contain Cargill Dow confidential information. Contact Jed Randall at 952-742-0453 or jed randall@cargilldow.com for more information.

\title{
3.1.2.4. Model for linear viscoelastic behavior of PLA blends
}

Most of the work conducted in this subtask has been published in the journal articles listed below. The abstracts of the papers follows.

A novel approach to modeling viscoelastic properties of thermoplastic polymer melts, with applications to polylactides. Janzen, Jay; Dorgan, John R.. Colorado School of Mines, Golden, CO, USA. Annual Technical Conference - Society of Plastics Engineers (2002), 60th(Vol. 1), 929-931.

\begin{abstract}
We illustrate the utility of the Havriliak-Negami (HN) functional form as a basis for modeling linear viscoelastic properties of polymer melts, including miscible blends. Parameters obtained by fitting rheometric data with the HN model are phys. interpretable and hence comprise useful yet compact and efficient summaries of linear viscoelastic behavior. Such parameter sets can also supplant discrete-line relaxation spectra as inputs for constitutive-equation models that predict non-linear properties from linearviscoelasticity measurements. Published results for various polylactide (PLA) melts are conveniently schematized using the approach we suggest.
\end{abstract}

Polylactides: rheology and processing of a commercial polymer from renewable resources. Dorgan, John R.; Janzen, Jay; Cicero, John.Chem. Eng. Dept., Colorado School of Mines, Golden, CO, USA. Polymeric Materials Science and Engineering (2002), 86 348-349.

\footnotetext{
Abstract

The rheol. of polylactide (PLA) is explored both exptl. and through math. modeling. Data for PLAs of various mol. architectures are discussed in the context of the HavriliakNegami model. It is demonstrated that the rheol. properties of interest are well described by this model and that accurate quant. predictions for blend properties can be made. In addn., the use of the resulting continuous distribution function arising from the model avoids truncation errors assocd. with more conventional approaches. Considerable improvement in predicting nonlinear rheol. properties is achieved. Implications regarding the processing of PLA fibers are discussed.
}

\subsubsection{Blends of PLA with other degradable polymers for property enhancement}


The following article, which will be submitted to Polymer Science and Engineering, contains the information on blends with other degradable polymers.

\title{
Blends of Poly(lactic acid) with a Degradable Aliphatic-Aromatic Polyester Tercopolymer, Bao, Lihong, Dorgan, John D., and Muzzy, John
}

\begin{abstract}
Polymers from renewable resources is a growing paradigm in the polymers industry. Recent developments have led to an expanding range of potential applications, especially in packing and fibers. Polylactides (PLAs) have been known for several decades and have recently gained considerable commercial significance. PLA is currently the most produced biobased polymer derived from agriculture resources through bioconversion and polymerization. Large scale of commercial production has already begun.
\end{abstract}

Extensive research work related to PLA synthesis, chemical, physicochemical and mechanical properties has been performed. Most of its properties are comparable to those of synthetic polymer from fossil-oil such as Polyethylene (PE), Polystyrene (PS), Polypropylene (PP) and Polyethylene terephthalate (PET). A comparison of mechanical properties among these polymers shows that PLA is much like Polystyrene, it has relatively high modulus but is brittle. Another potential problem with PLA is that it has reasonably high polarity that can cause adhesive problems with certain substrates leading to processing difficulties.

To improve mechanical properties and processibility of PLA and retain its biodegradability, blending of PLA with other biodegradable polymer is logical. Biodegradable polyesters generally work well in blends with PLA, starch, and naturalfiber reinforcements. A broad range of synthetic biodegradable resins based on aliphatic polyesters and aliphatic-aromatic copolyesters have been commercialized. Eastar® Bio polyesters from Eastman Chemical Company are a family of aliphatic-aromatic copolyesters based on butanediol, adipic acid, and terephthalic acid. Eastar Bio GP (general purpose) has a melting point of $108 \mathrm{oC}$ and offers good contact clarity and adhesion along with high elongation to break (up to $800 \%$ ). This material also possesses high moisture and grease resistance while processing much like LDPE. Shortcomings for some applications include relatively low stiffness, poor melt strength, and a tendency to stick in injection molds. Such synthetic aliphatic-aromatic biodegradable polyesters (BASF makes a similar commercial product called EcoFlex) tend to complement the properties of PLA and are a logical choice for improving mechanical properties through blending while retaining environmental degradability.

The objective of this article is to investigate the properties of PLA/Eastar BioGP blends. Data on the melt rheological properties, mechanical properties, and gas (CO2) permeation are presented.

\subsubsection{Extensional Viscosity Measurements}

Isothermal extensional viscosity measurements of PLA optical copolymers were examined and reported in the following journal article:

Melt Rheology of High L-content Poly(lactic acid) - I.L. Palade, H.J. Lehermeier, and J.R. Dorgan, Macromolecules, 2001, 34, 1384- 1390. 


\begin{abstract}
In this paper, the first data on the nonlinear extensional flow behavior of PLA are reported. In addition, new findings regarding the linear viscoelastic properties are also presented. The melt rheology of linear poly(lactic acid)s (PLA) characterized by a high content of the $L$ form of the monomer is comprehensively investigated. Measurements of dynamic, steady, and transient shear viscosities are presented. Extensional data on PLA are presented for the first time and show a strong strain hardening behavior. The Cox-Merz relationship is obeyed over a particularly wide range (roughly three decades of shear rate). Results for high molecular weight samples suggest that the plateau modulus is approximately $5 \times 10^{5} \mathrm{~Pa}$. In addition, the zero shear viscosity, $\eta_{\mathrm{o}}$, for these materials is found to roughly scale with the expected 3.4 power versus molecular weight. The transient shear results are satisfactorily predicted using a truncated form of the KBKZ constitutive equation and a set of Maxwell modes $\left(G_{k}, \lambda_{k}\right)$ derived from the dynamic spectra. However, to capture the observed extensional hardening, an additional long time relaxation mode must be added to the spectrum. Time sweep measurements demonstrate that the melt stability of the polymer precludes long time measurements. Chemical changes manifest themselves in a lack of adherence to the principle of timetemperature superposition. It is shown that stabilization of poly(lactic acid) using tris(nonylphenyl) phosphite is possible and leads to a material that is thermorheologically simple within the experimentally assessable rates of deformation.
\end{abstract}

\title{
3.1.4. Crystallization studies
}

\subsubsection{Effects of optical composition on PLA quiescent crystallization behavior}

The quiescent crystallization of slow crystallizing PLA ( 4-5\% D isomer) was studied. Studies comparing crystallization by cooling from the melt to isothermal crystallization temperature vs. rapidly quenching and heating to the isothermal crystallization temperature were conducted. The rate of crystal nucleation was strongly influenced by these two modes of crystallization, with the rapid quench resulting in a much greater nucleating density.

The effects of meso-lactide and D-lactide optical impurities in L-lactide rich PLA polymers on melting point and lineal crystal growth rate was examined in detail.

The studies utilize and contain Cargill Dow confidential information. Contact Jed Randall at 952-742-0453 or jed randall@cargilldow.com for more information.

\subsubsection{Quiescent crystallization of PLA with nucleating agent}

Crystallization studies utilizing nucleating agents were conducted. The studies utilize and contain Cargill Dow confidential information. Contact Jed Randall at 952-742-0453 or jed randall@cargilldow.com for more information.

\subsubsection{Processing studies}

\subsubsection{Development of a thermoforming process for crystalline PLA}

The focus of this project was to develop articles with higher heat resistance, in an economically viable thermoforming process. This was accomplished by the development of a novel thermoforming process that utilizes composition and process 
variables that facilitate crystallization of the PLA during processing. Crystalline PLA (cPLA) applications studies included by deep draw (surface area draw ration >1.5: 1) and shallow draw applications (<1.5: 1$)$.

Multiple shallow draw cPLA articles were successfully been thermoformed at commercial speeds on commercial lines, and were later introduced into markets.

There is an opportunity for tolerating down gauging with cPLA articles as compared to incumbent materials, i.e. polypropylene and polystyrene, while still maintaining the integrity and robustness of the thermoformed articles. As noted below, the tensile storage modulus (E') of cPLA is significantly higher than that of PS and PP below the $T_{g}$, and at elevated temperatures (figure 1). In an effort to reduce final part cost, thermoformed articles have been down gauged up to $25 \%$ as compared to the incumbent material with successful results.

Figure 1.

Storage Modulus (Tensile) VS Temperature

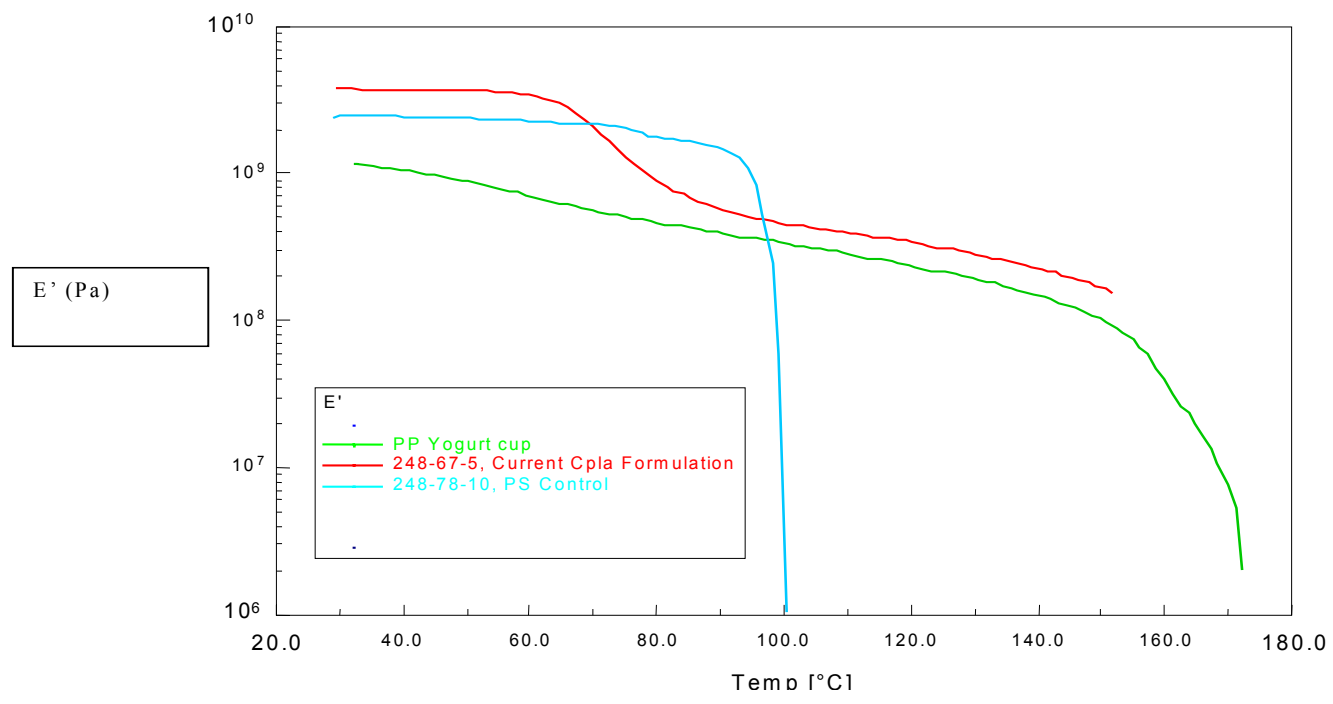

The process and compositions utilize and contain Cargill Dow confidential information. Contact Jed Randall at 952-742-0453 or jed randall@cargilldow.com for more information.

\subsubsection{Development of a stretch injection blow molding process for crystalline PLA}

In addition to the work shown below, processing studies for thermoforming, and injection stretch blow molding (ISBM) were also performed. Processes and formulations were developed that enhance the thermal dimensional stability of thermoforms. The process has made significant process with regard to commercial speeds, and end use targets.

For ISBM, formulations and processes were developed to enhance the processing and end use characteristics of bottles. The studies utilize and contain Cargill Dow confidential information. Contact Jed Randall at 952-742-0453 or jed randall@cargilldow.com for more information. 


\title{
3.1.5.3. Fiber spinning studies
}

Most of the work conducted in this subtask has been published in the journal articles listed below. The abstracts of the papers follow.

Effects of molecular architecture on two-step, melt-spun poly(lactic acid) fibers. Cicero, John A.; Dorgan, John R.; Garrett, James; Runt, James; Lin, J. S. Department of Chemical Engineering, Colorado School of Mines, Golden, CO, USA. Journal of Applied Polymer Science (2002), 86(11), 2839-2846.

\begin{abstract}
Fibers of poly(lactic acid) (PLA) produced by two-step melt spinning have been studied. The PLA resins used contain a 96:04 ratio of L:D stereochem. centers; however, one of the materials is branched by a peroxide treatment. The thermal, mech., and morphol. properties of the fibers are compared for the two different mol. architectures. In the branched material, at least some of the branches exceed the entanglement mol. wt. The branched material is accordingly characterized by greater shear and extensional viscosity than the linear material. Fiber properties are highly influenced by the draw ratio; both branched and linear materials reach a plateau of about $35 \%$ crystallinity. The branched polymer reaches the plateau at a lower draw ratio, and this is indicative of faster crystn. kinetics. Both materials shrink in boiling water, and the amt. of shrinkage decreases with increasing draw ratio. At an intermediate draw ratio of 6 , the branched material is characterized by significantly larger shrinkage. With small-angle X-ray scattering and at. force microscopy, the morphol. is found to be fibrillar. Microfibril diams. range from approx. 20 to $30 \mathrm{~nm}$ and are almost identical for the two mol. architectures studied.
\end{abstract}

Supramolecular morphology of two-step, melt-spun poly(lactic acid) fibers. Cicero, John A.; Dorgan, John R.; Janzen, Jay; Garrett, James; Runt, James; Lin, J. S. Department of Chemical Engineering, Colorado School of Mines, Golden, CO, USA. Journal of Applied Polymer Science (2002), 86(11), 2828-2838.

\begin{abstract}
Fibers of poly(lactic acid) produced by two-step melt spinning were studied. The morphol. is elucidated with respect to the thermal and mech. properties of fibers produced at cold-draw ratios of 1-8. With at. force microscopy and small-angle $x$-ray scattering, a fibrillar morphol. is found, with microfibril diams. ranging from $30-60 \mathrm{~nm}$. Shrinkage properties indicate that, with increasing draw ratio, the fibers undergo a transition from class 2 to class 1 within the classification proposed by Keller. A supramol. model for the morphol. of the fibers is presented that entails a highly oriented skin with a core consisting of microfibrils. The orientation of the cryst. blocks within the microfibrils is similar to what was reported for nylon fibers.
\end{abstract}

Phosphite stabilization effects on two-step melt-spun fibers of polylactide. Cicero, John A.; Dorgan, John R.; Dec, Steven F.; Knauss, Daniel M. Department of Chemical Engineering, Colorado School of Mines, Golden, CO, USA. Polymer Degradation and Stability (2002), 78(1), 95-105.

\section{Abstract}

The effects of mol. wt. stabilization on mech. properties of polylactide (PLA) fibers are investigated. The textile-grade PLA contains a 98:02 ratio of L:D stereocenters and fibers are produced by the 2 step method, involving a primary quench and cold drawing. 
Mol. wt. loss, which is approx. $30 \%$ for unstabilized PLA, can be eliminated by the addn. of small amts. of tris(nonylphenyl) phosphite prior to processing. The thermal and mech. properties of fibers produced with 2 different concns. of TNPP are compared to those of unstabilized PLA. Faster crystn. rates are obtainable with addn. of the stabilizer, but final crystallinities are unaffected. Mech. properties of the TNPP-enriched fibers are greatly improved over their unstabilized counterparts. Tensile strengths can be improved by $10-30 \%$ at a given draw ratio, while modulus may be improved by $10-25 \%$. Excessive amts. of TNPP or insufficient mixing result in inhomogeneities that are deleterious to mech. properties. Based on the available information, chain extension is believed to be the most likely mechanism for the mol. wt. stabilization.

Physical Properties and Fiber Morphology of Poly(lactic acid) Obtained from Continuous Two-Step Melt Spinning. Cicero, John A.; Dorgan, John R..

Department of Chemical Engineering, Colorado School of Mines, Golden, CO, USA. Journal of Polymers and the Environment (2002), Volume Date 2001, 9(1), 1-10.

\begin{abstract}
Fibers of poly(lactic acid) (PLA) produced by two-step melt-spinning are studied. The PLA resin used contains a 98:02 ratio of $\mathrm{I}: \mathrm{d}$ stereochem. centers. A range of processing conditions is explored. The cold-draw ratio is varied from 1 to 8 under conditions of const. heating. In addn., three draw ratios are studied at three different heating rates. The thermal, mech., and morphol. properties of the resultant fibers are detd. Properties can be widely manipulated through a combination of draw ratio and draw temp. A max. tensile strength and modulus of $0.38 \mathrm{GPa}$ and $3.2 \mathrm{GPa}$, resp., are obtainable. Using at. force microscopy, the fiber morphol. is found to be highly fibrillar; microfibril diams. are roughly $40 \mathrm{~nm}$ in diam. Very high draw ratios cause the fiber to turn from shiny and translucent to dull and white; this transition is attributed to surface crazing. Significant mol. wt. loss is obsd. upon processing (wt.-av. mol. wts. drops between $27 \%$ and $43 \%$ ).
\end{abstract}

\title{
3.1.6. Development of a predictive model
}

Developing a predictive model to describe the behavior of PLA so that new formulations specific for emerging applications can be created is a challenging and ongoing endeavor. Significant progress was made in the following two areas:

\subsubsection{Linear PLA rheology model}

A model describing the flow characteristics for PLA was developed and transferred from Colorado School of Mines to Cargill Dow.

\subsubsection{Rheology blend model}

A model describing the flow characteristics for blends of linear or branched PLA was developed and transferred from Colorado School of Mines to Cargill Dow.

\subsection{Interfacial Engineering}

\subsubsection{Preparation of Model Paper and Cardboard Surfaces and Adhesion studies of PLA laminated to paper substrates}

A series of PLA/paper composites were prepared to evaluate the adhesion properties of PLA and the effects of different paper substrates. PLA films prepared at NREL and films 
from Cargill Dow were both evaluated. The paper substrates were softwood Kraft papers (bleached and unbleached) obtained from Rayonier.

Adhesion was measured with 180-degree peel tests. These tests measure the force required to pull the film from the paper substrate. The measured force is highly variable as shown in Figure 2. These four samples are replicates indicating the high variability within and between samples. These differences are due, in part, to the roughness of the paper surfaces. The individual paper fibers can adhere to the PLA films, but gaps between the fibers can leave voids that have very low strength.

The peel strength can be measure by several techniques, including the area under the force elongation curve, averaging the highest force peaks or the difference between the peak maximum and minimum. All of these routines were used to estimate the peel strength of the PLA/paper composites. The effects of time and temperature used to "bond" the PLA films are shown in Figure 3. There were significant scatter in the measured peel strength but there was a general trend of increased strength with increased press times and the strength reached a maximum at $90^{\circ} \mathrm{C}$. The peel strength decreased above $90^{\circ} \mathrm{C}$, which is likely due to flow of the film into the paper substrate.

Peel Tests on Replicates

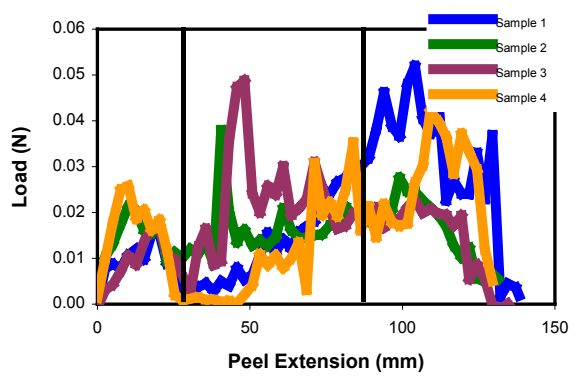

Figure 2. Force-extension plots for the paper/PLA composites. This plot highlights the scatter within and between different samples prepared under the same conditions.
Effect of Temperature and Press Time

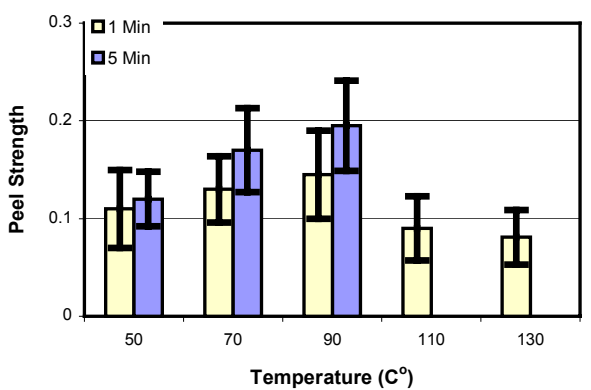

Figure 3. Effects of press temperature and time on the measured peel strength of bleached kraft paper and untreated PLA film.

The effects of different paper substrates on the peel strength were also measured. Bleached and unbleached Kraft papers have significant differences in their chemical composition, especially the composition at the paper surface. Unbleached paper will have low molecular weight, amorphous lignin and hemicellulose redeposited on the surface on the paper. The bleached Kraft will have essentially no lignin and very low levels of hemicellulose on the paper surface. The bleached Kraft will also have some crystalline cellulose at the surface. The effects of the different paper substrates are shown in Figure 4. Again, there is a great deal of scatter in the measured peel strength, but there is a consistent trend of higher peel strength for the unbleached Kraft samples. This is likely due to the presence of the amorphous lignin and hemicelluloses at the surface of the unbleached paper. In particular, the phenolic hydroxyls in the lignin are known to form strong hydrogen bonds with esters groups in polymers. 


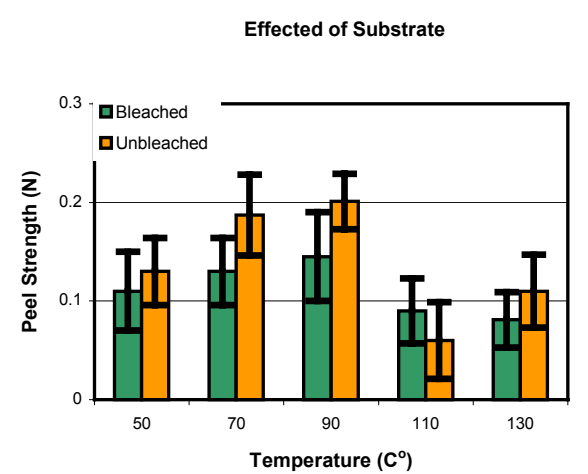

Figure 4. The effect of the source on peel strength.
Topography, NOSB.HDF

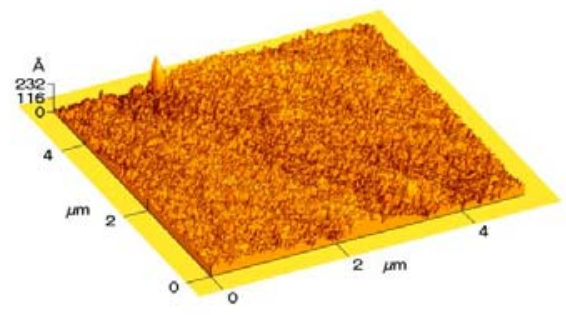

Figure 5. AFM image of untreated PLA film. The untreated films were the smoothest films. The image is 5 microns on a side.

There are two concerns about the results of these peel tests. The first is the significant scatter within and between samples prepared under nominally the same conditions. There are also significant differences between the processes used to prepare the PLA/Paper samples at NREL, e.g., relatively long times and low pressure, and the commercial processes used to prepare coated paper. The commercial process is very high speed, hundreds of feet per minute, and the rheology and heat transfer processes are very different. So while the goal of this task was to study the effects of the paper surface, the scatter and processing differences made these results less useful.

\subsubsection{Preparation of Model Metal Surfaces and Characterization of PLA films and aluminum coatings}

Cargill Dow to NREL supplied films of various surface treatments for surface chemistry and topography analysis. Surface treatments included corona, flame, plasma and chemical vapor deposition (CVD). Differences in surface chemistry, water contact angle, dyne level and surface roughness were observed. The studies utilize and contain Cargill Dow confidential information. Contact Jed Randall at 952-742-0453 or jed randall@cargilldow.com for more information on the specifics of the treatments.

\section{Initial Evaluation of PLA Films}

The four PLA films samples sent by Cargill Dow LLC were evaluated with Atomic Force Microscopy (AFM). The samples included an untreated film, and films subjected to corona discharge, flame treatment and plasma treatment. Samples from two different films were submitted for analysis and 4-6 areas on each film were evaluated. The AFM images showed significant differences between the different films. In some cases there were also significant differences between and within the films.

The AFM images shown in Figures 5 through 8 highlight differences between the untreated and treated films. The film shown in Figure 5 is the untreated film and is a relatively smooth film. Figure 6 shows the AFM image of the Corona treated film, which was relatively smooth. The film in Figures 7 and 8 are plasma treated. These films have both smooth regions and the roughest of the films' regions. Finally, Figure 9 shows the AFM image flame treated film, which shows evidence of redeposition of particles. 
Differences between the films were quantified and are shown in Table 1. Both of the untreated films are relatively smooth. Both of the Corona discharge treated films were relatively smooth, although the second film had one area with high roughness. The two plasma treated films were very different from one another. One film was very smooth and the second was the roughest film examined. The flame treated films were both relatively rough and, as seen in Figure 9, shows evidence of redeposition of material on the surface.

Topography. NO3B. HDF

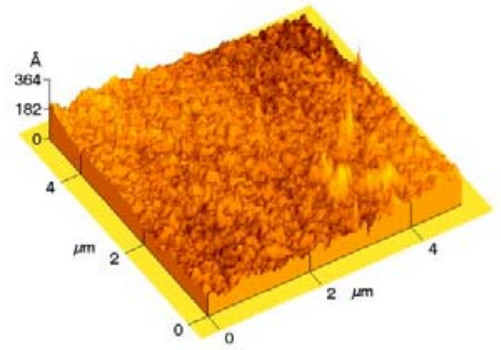

Figure 6. AFM image of corona treated PLA film. These films were relative smooth. The image is 5 microns on a side.

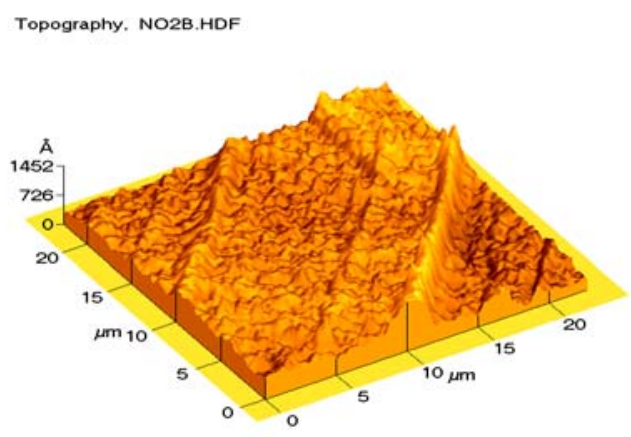

Figure 8. AFM image of the "rough" plasma treated PLA film. The image is 5 microns on a side.

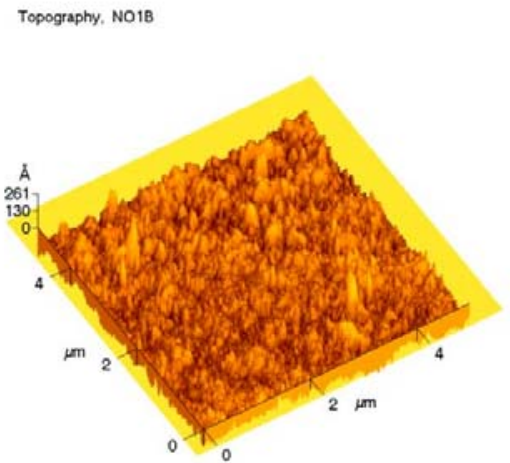

Figure 7. AFM image of the "smooth" plasma treated PLA film. The image is 5 microns on a side.

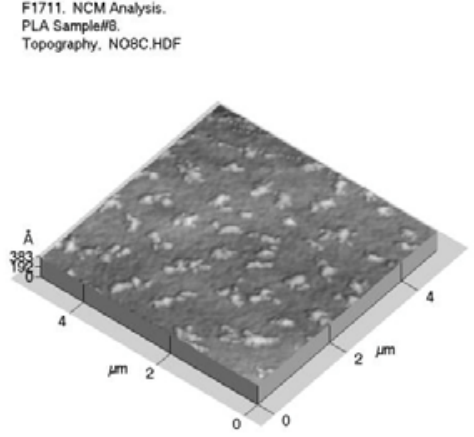

Figure 9. AFM image of flame treated PLA film. The films show evidence of redeposition particles. The image is 5 microns on a side.

\begin{tabular}{|l|l|l|l|l|}
\hline \multicolumn{3}{|c|}{ Table 1. Summary of the surface roughness of different PLA films measured with AFM. } \\
\hline & $\begin{array}{l}\text { Untreated } \\
\text { PLA }\end{array}$ & $\begin{array}{l}\text { Corona } \\
\text { Discharge }\end{array}$ & $\begin{array}{l}\text { Plasma } \\
\text { Treatment }\end{array}$ & $\begin{array}{l}\text { Flame } \\
\text { Treatment }\end{array}$ \\
\hline Film replicate 1 & 18 Ang. RMS & 15 Ang. RMS & 38 Ang. RMS & 150 Ang. RMS \\
\hline Film replicate 2 & 16 Ang. RMS & $\begin{array}{l}\text { 30 Ang. RMS } \\
\text { (one rough } \\
\text { area) }\end{array}$ & 250 Ang. RMS & 150 Ang. RMS \\
\hline
\end{tabular}

Second evaluation of films and metal surfaces 
For the studies of the interactions between PLA films and metal surfaces NREL was asked to focus on characterization of the PLA treated in different ways. Cargill Dow LLC provided a set of five PLA films. These films included a control and films modified with five different treatments: Corona, Flame, two types of Plasma and Chemical Vapor Deposition (CVD). All of these films were prepared under commercial conditions by Cargill Dow LLC's partners.

In an initial evaluation the PLA films were subjected to Differential Scanning Calorimetry (DSC) to determine the properties of the base PLA. This analysis shows that the films came from three different grades of PLA. There is a $20^{\circ} \mathrm{C}$ difference in the melting point for the different films. Representative scans are shown in Figure 10. There are clearly differences between the Corona and Flame treated samples compared to the Plasma treated samples. The CVD treated sample is also different than the other two sets of samples. The films vary in lactic acid L:D ratio which results in the changing melting point.

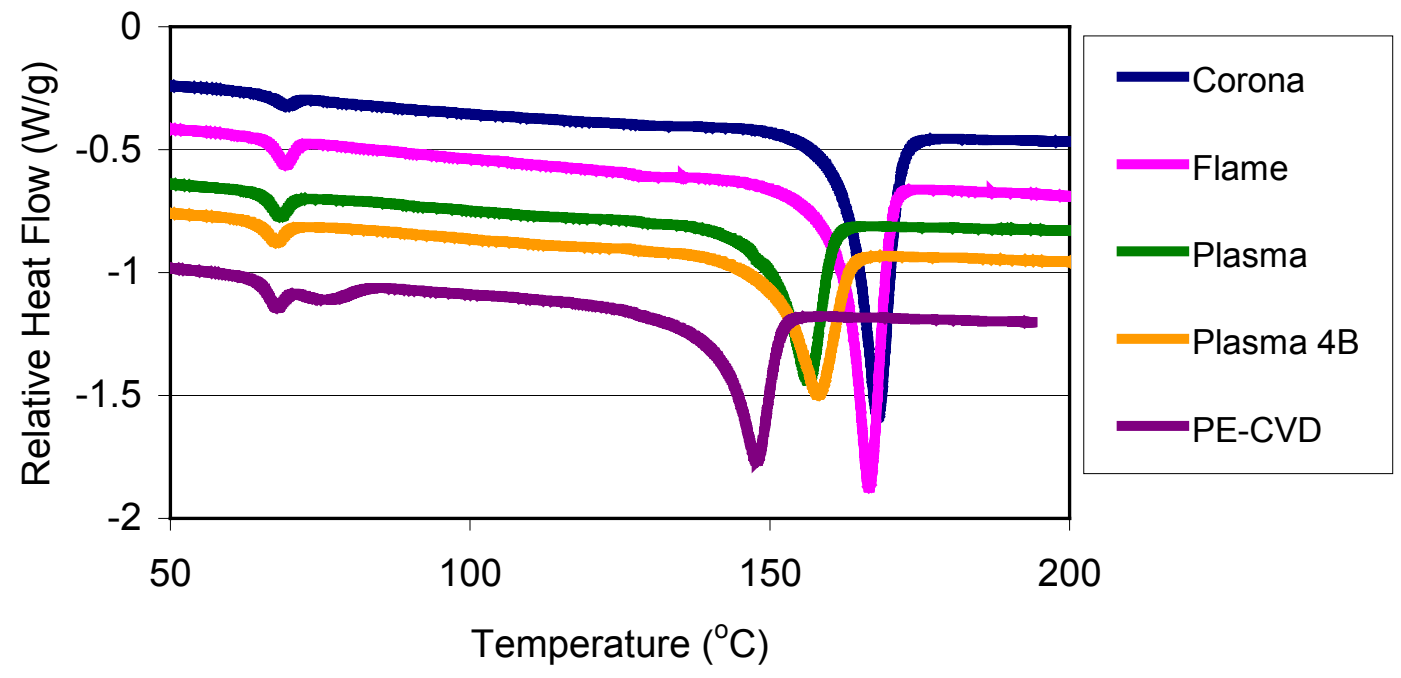

Figure 10. DSC thermograms of the treated PLA films.

These differences in the composition of the PLA films complicate the interpretation of the effects of different surface treatments.

These films were subjected to X-ray Photoelectric Spectroscopy (XPS) analysis using a Phi 670 XPS system. All spectra were acquired from the as-received samples. The pass energy for the high resolutions scans of the $\mathrm{C} 1 \mathrm{~s}$ lines was $5.85 \mathrm{eV}$. The $\mathrm{x}$-rays were incident at 45 degrees from the surface normal, and the takeoff angle of the photoelectrons was the same. A small $\left(4 * 4 \mathrm{~cm}^{2}\right)$ sample was cut for the film, placed in the XPS vacuum chamber, an initial survey scan was run, followed by one to three high resolution scans. Then a second area of the same small sample was run. Charging was a minor problem for all the samples.

The XPS survey scan provides an overall evaluation of the surface. Specifically, the survey can be used to measure the carbon to oxygen ratio and detect the presence of other elements on the surface. Figure 11 shows the survey scan for the control films. The results of these survey scans are shown in Table 2. There are clear changes in the carbon to oxygen ratio. The control, corona, flame and one plasma treated samples are 
all similar with a $\mathrm{C} / \mathrm{O}$ of about 1.6 , which is similar to the $\mathrm{C} / \mathrm{O}$ ratio of 1.71 reported in the literature (Ma et al, 2002). Two treatments showed significant changes in the overall composition of at the surface. Sample 329-36-4 (Plasma 4B) had a significant increase in the carbon to oxygen ratio while sample 329-36-5 (CVD-PE) had a significant decrease in the carbon to oxygen ratio. The survey scans also showed the presence of nitrogen and aluminum in the plasma treated samples and silicon in the CVD-PE treated sample (Figure 12). The presence of these elements also makes the water contact angle results somewhat questionable.

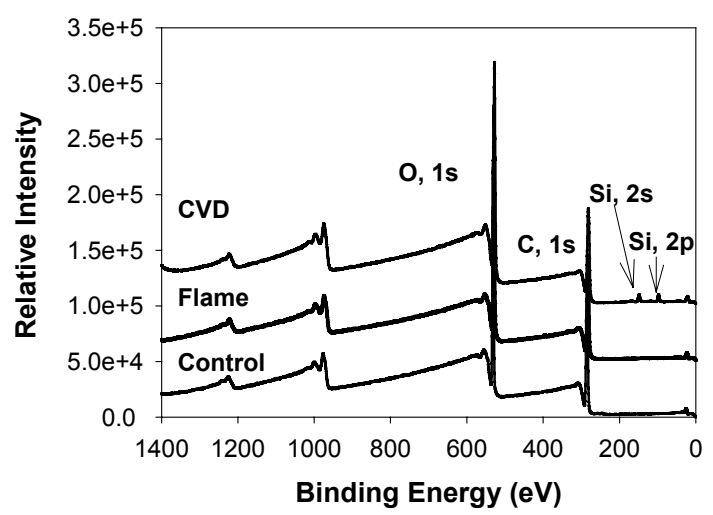

Figure 11.

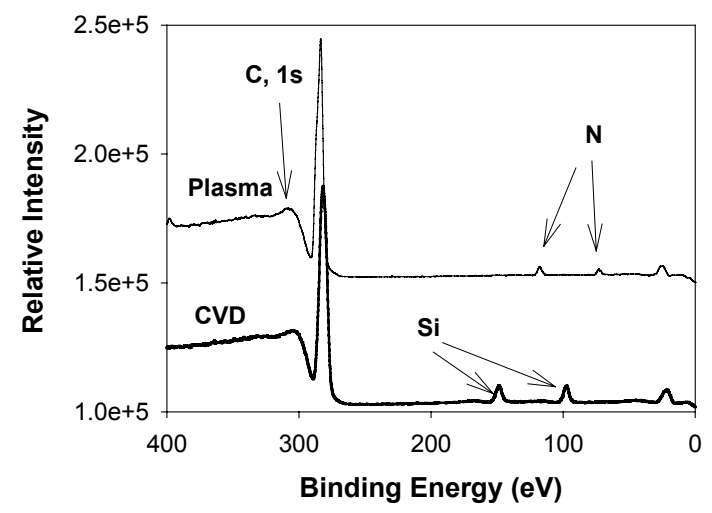

Figure 12. 
Table 2. XPS and WCA Results

\begin{tabular}{|l|l|l|l|l|l|l|l|}
\hline Surface Treatment & & & & & & & \\
\hline & & C1s & O1s & N1s & Al2p & Si2p & WCA \\
\hline $\begin{array}{l}\text { Control (329-36-1 Back } \\
\text { side) }\end{array}$ & Ave & 61 & 39 & ND & ND & ND & 68.6 \\
& Std Dev 0.7 & 0.7 & - & - & - & 1.9 \\
Corona (329-36-1) & Ave & 60.8 & 39.2 & ND & ND & ND & 63.2 \\
& Std Dev & - & - & - & - & - & 1.1 \\
Flame (329-36-2) & Ave & 61.6 & 38.4 & ND & ND & ND & 58.1 \\
& Std Dev 1.1 & 1.1 & - & - & - & 3.6 \\
Plasma (329-36-3) & Ave & 60.8 & 36.9 & 0.9 & 1.4 & ND & 60.9 \\
& Std Dev 0.6 & 0.6 & 0.05 & 0.5 & - & 2.5 \\
Plasma 4B (329-36-4) & Ave & 65 & 36.9 & 1.2 & ND & ND & 59.6 \\
& Std Dev 0.4 & 0.3 & 0.05 & - & - & 2.3 \\
PE-CVD (329-36-5) & Ave & 50.7 & 44.3 & ND & ND & 5 & 81.8 \\
& Std Dev 5 & 2.2 & - & - & 2.8 & 2.1 \\
\hline
\end{tabular}

ND $=$ None Detected

The high resolution XPS scans of the S1 carbon also showed significant differences between the samples. Curve fitting the high resolution scans provides the ratio of the different types of carbon, e.g., $\mathrm{CH}, \mathrm{CO}$ or OCO. The control sample is different than all the treated samples. The control samples are shown in Figure 13. The high resolution XPS scans of the S1 carbon from the Corona and Flame treated samples were similar and different than the Control, Figures 14 and 15, respectively. The Plasma treated films were different than the Control films and the two different plasma treatments do not appear to differ significantly from one another (Figure 16). Figure 17 shows high resolution XPS scans of the S1 carbon for the CVD treated film, which was somewhat similar to the Corona and Flame treated films. The CVD films also exhibited problems with reproducibility; three scans are not included in Figure 17.

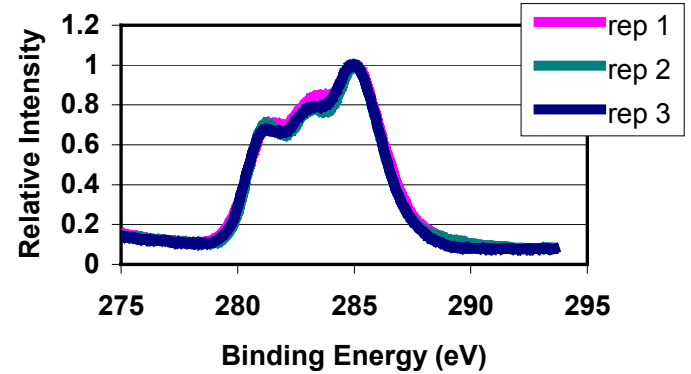

Figure 13. High resolution XPS scan of the

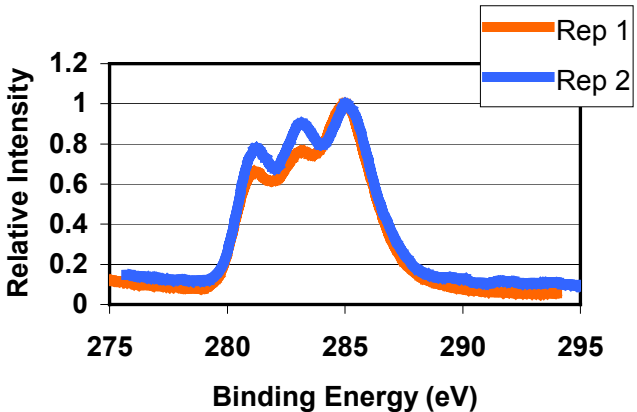

Figure 14. High resolution XPS scan of the 


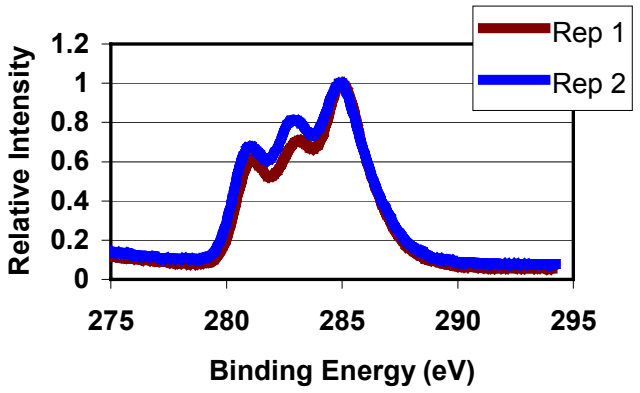

Figure 15. High resolution XPS scan of the carbon 1s peak of the flame treated PLA films.

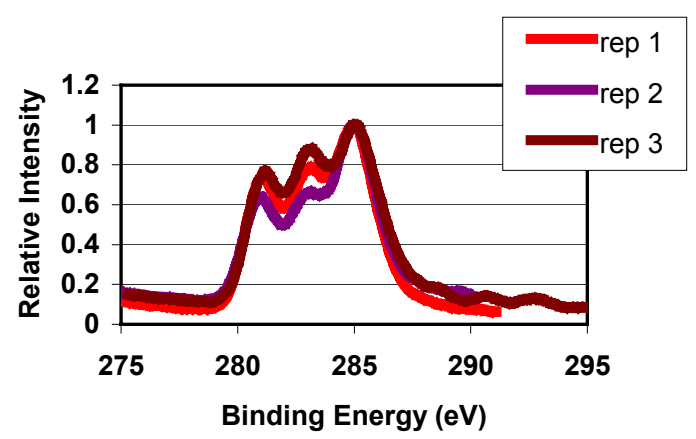

Figure 17. High resolution XPS scan of the carbon 1s peak of the CVD treated PLA films.

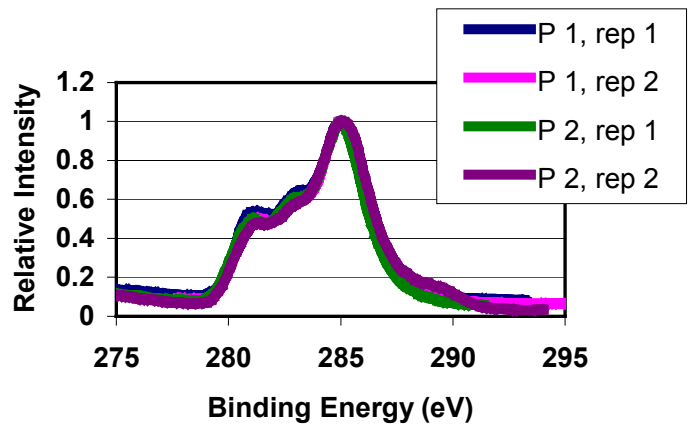

Figure 16. High resolution XPS scan of the carbon 1s peak of the plasma treated PLA films.

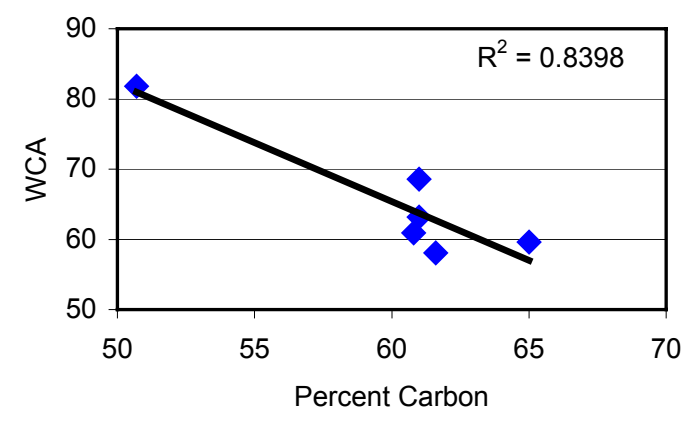

Figure 18. Relationship between the water contact angle and the relative intensity of the $\mathrm{CH}$ peak.

The water contact angle (WCA) of the films was also measured. Two pieces of film from each sample were measured 5 times, for a total of 10 replicate measurements. The results from the WCA experiments are also shown in Table 2. The WCA for the control is about $69^{\circ}$, which is similar to WCA measurements reported in the literature. There are no significant differences between in WCA between the Corona, Flame and Plasma treated films, and all of these films have a WCA that is lower than the control. The CVD treated film is much higher than the control, but it is important to note that the XPS analysis also showed that the CVD films have Si on the surface.

AFM analysis of these films was also conducted. The AFM scans showed significant differences among the surface-treated films.

These AFM scans of this second set of films were also quite different than those obtained for the first set of films. Several representative images are shown in Figure 19. 


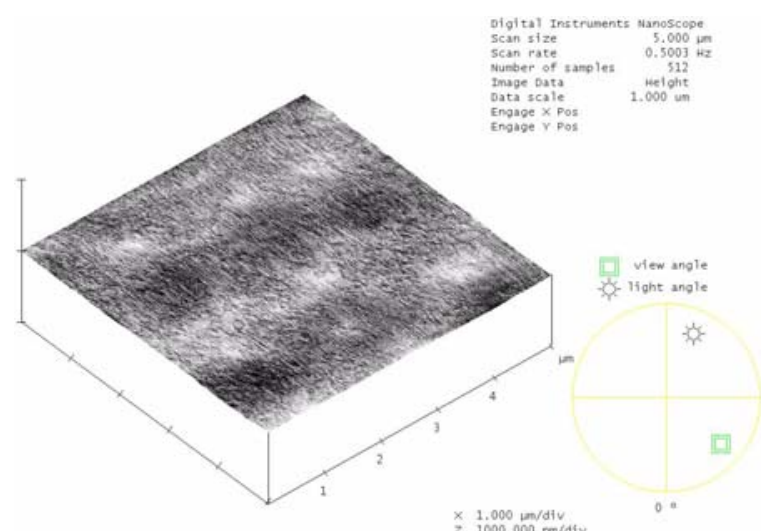

untreated.

Control

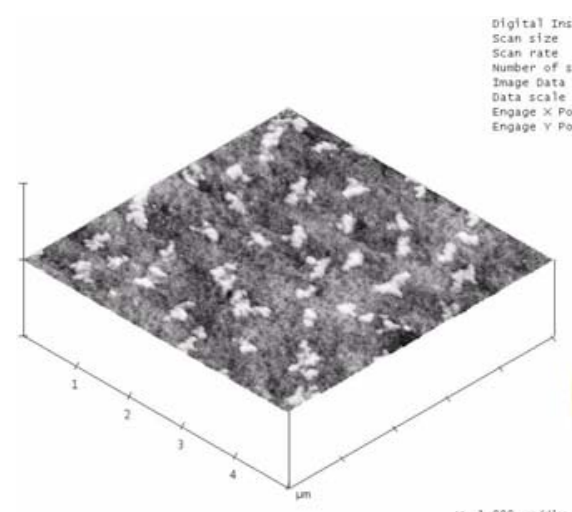

$\underset{z}{\times} \quad \begin{aligned} & 1.000 \\ & 5000\end{aligned}$

32936-2b.001

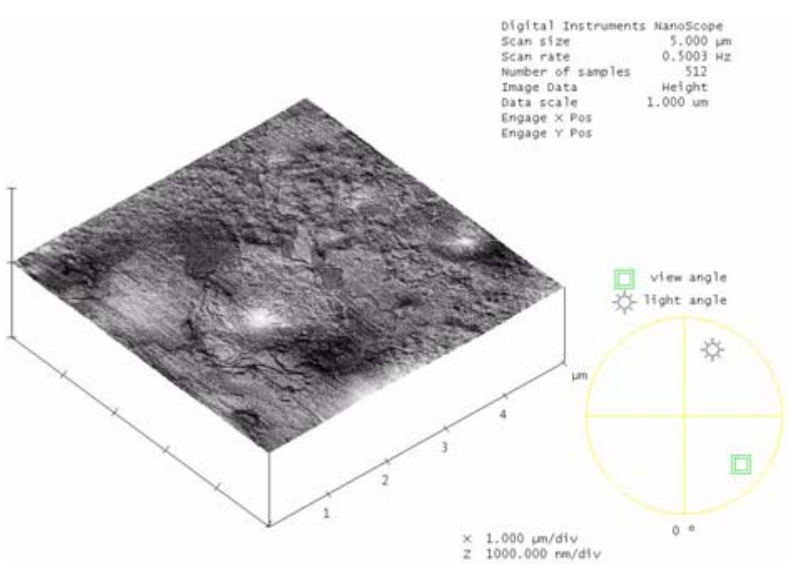

corona Treated.

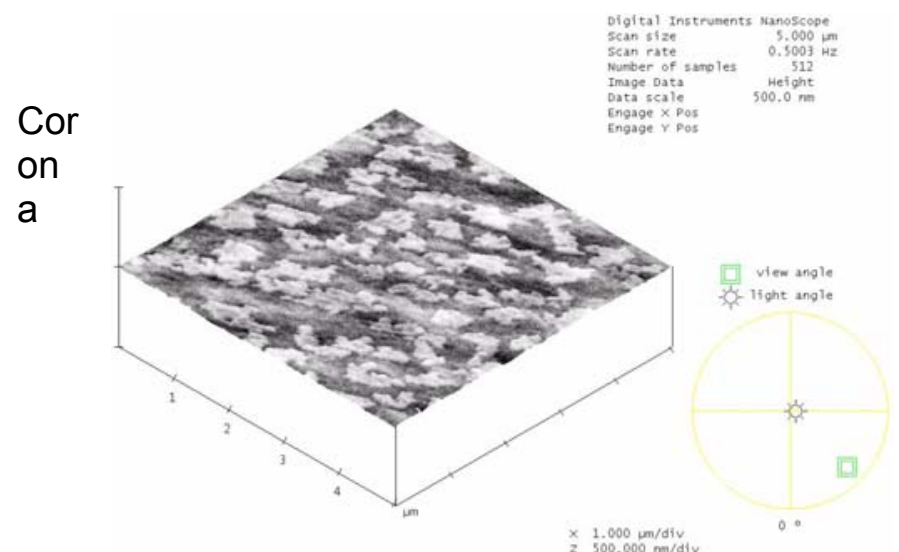

32936-36.001
Flame

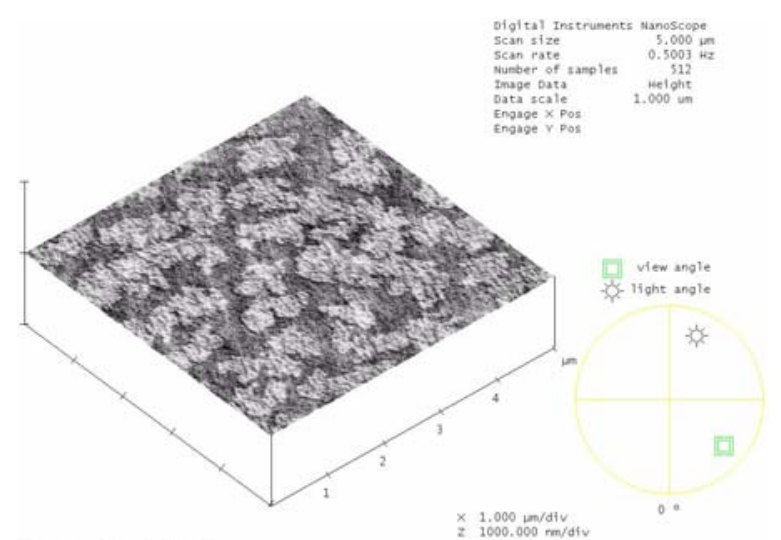

Plasma Treated.
$329-16-4.002$
Plasma (I)

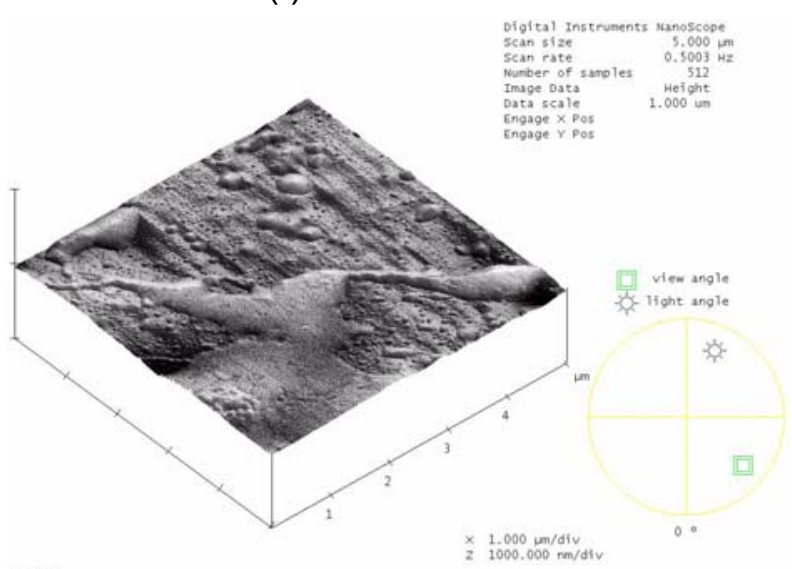

PE-CVO. 
Figure 19. Representative AFM images of Control and surface-treated films

While the Control film appears relatively smooth, the two plasma-treated films and the flame-treated film appear significantly rougher or "etched', with the flame-treated sample having a distinctly different roughness pattern than the plasma-treated films. The Corona treated film shows only minor changes from the Control. In contrast, the CVD film is notably different from all other films with what appears to be small (submicron) areas of a second phase pooled on the top of the film.

Differences between the films in terms of Peak-to-Peak depth, Maximum Depth (Depth $_{\text {Max }}$ ) and Root Mean Square Roughness (RMS) were quantified and are shown in Table 3. To provide for a robust evaluation, a total of 8 individual samples (2 replicates from 4 films) from each treatment were analyzed. AFM depth analysis measures the height difference between two dominant features that occur at distinct heights.

Specifically, Peak-to-Peak measures the average depth between two peaks, while Depth $_{\text {Max }}$ measures the maximum roughness height within a sample length. The results show that flame and corona surface treatments did not have a significant impact on surface roughness, but both plasma and CVD treatments did significantly increase the roughness. CVD treatment produced the roughest films examined in this film series.

Table 3. AFM Surface Profile Parameters

\begin{tabular}{rllllll}
\hline \multicolumn{7}{c}{ Control Corona Flame Plasma I Plasma IIPE-CVD } \\
\hline Peak to Peak (nm) & & & & & & \\
Ave: & 0.38 & 0.25 & 0.34 & 0.50 & 0.77 & 1.25 \\
StDev: & 0.30 & 0.17 & 0.18 & 0.29 & 0.93 & 0.63
\end{tabular}

DepthMax (nm)

\begin{tabular}{|c|c|c|c|c|c|}
\hline Ave: & 28 & 23 & 34 & 37 & 31 \\
\hline StDev: & 14 & 11 & 12 & 17 & 17 \\
\hline
\end{tabular}

RMS Roughness

$(\mathrm{nm})$

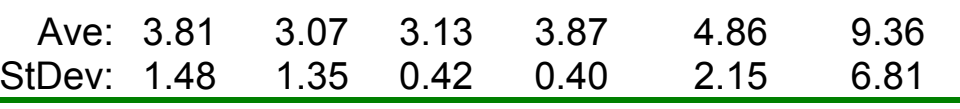

Representative histograms that display the distribution of the data are shown in Figure 20 for each of the films. Histogram \% reveals the percentage of the total number of points at the depth indicated by the cursor in the correlation histogram. Note the bimodal behavior of the plasma-treated films and, to a much lesser extent, the flametreated film. While this was observed in most of the replicate plasma and flame films, it was not seen in any of the other films. This points again to the fact that the plasma and flame-treated samples have a distinctly different roughness pattern than the other films. 

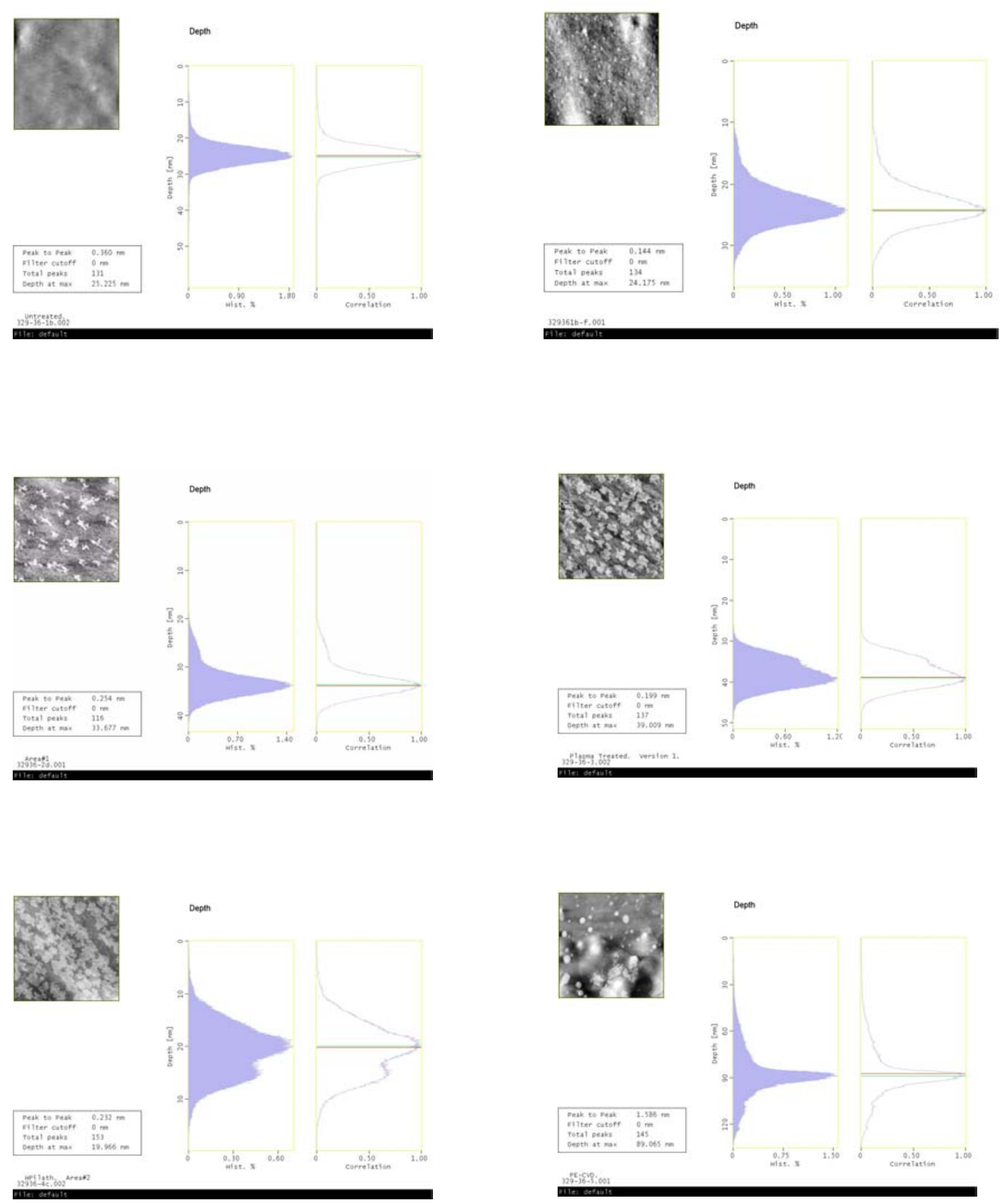

Figure 20. AFM Histograms of the various surface-treated films. From left to right, top to bottom: Control, Corona, Flame, Plasma I, Plasma II and CVD 
The histograms below are of two different samples, both plasma-treated, which show the variance in profile values of films having the same surface treatments while visually appearing quite similar. The reasons for this are varied and would require further analysis. It should be noted that this discrepancy between replicates was observed to the greatest extent with the Plasma II and CVC samples.
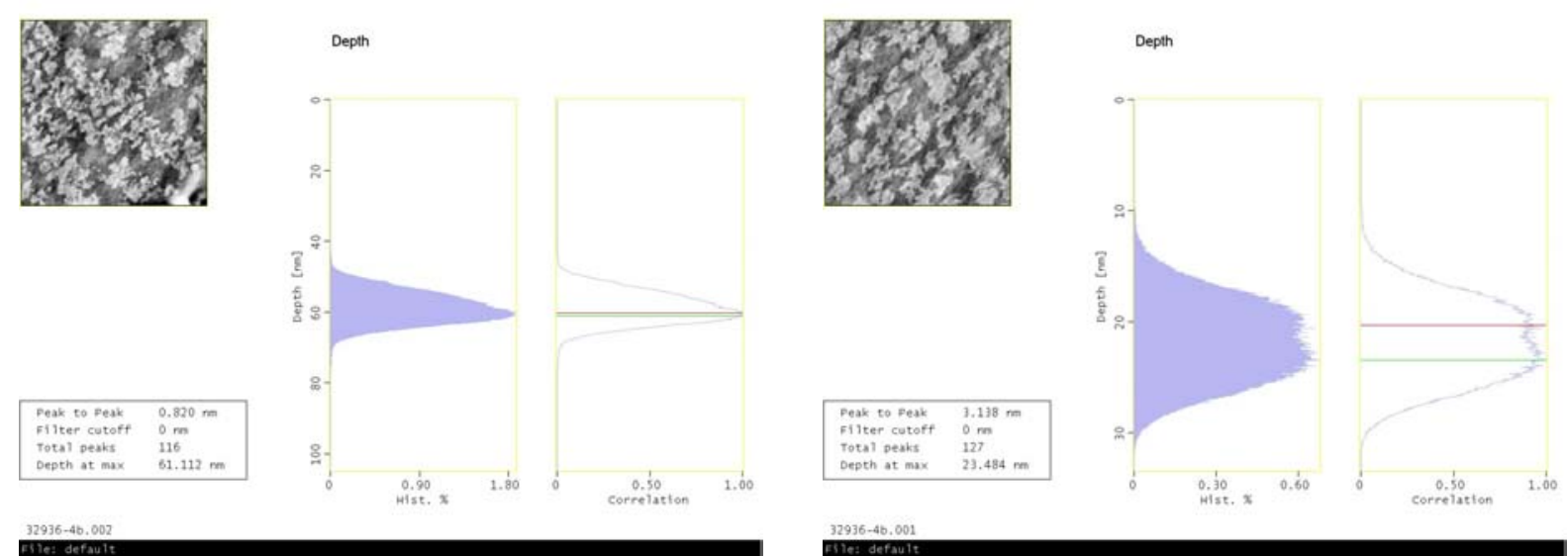

Figure 21. Variation in AFM Histogram values of two different Plasma II-treated films

Additional work was carried out on samples of metallized and unmetallized PLA films provided by Cargill Dow. The films were corona-treated, with and without aluminum metallization. The films consisted of a coextruded bilayer structure that had been biaxially oriented. The interest here was in examining the pinholes and scratches, and determining the root cause of the defects. Since a fumed silica anti-block agent was used in one of the layers film, it was thought that the anti-block agent might be pushing through both layers and causing roughness of the films.

Scanning Electron Microscopy (SEM) was used to examine overall surface structure and defeats. The SEM was manufactured by RJ Lee/Aspex Instruments and utilized a Titanium filament with a drive voltage of $15 \mathrm{keV}$.

The results do not show a clear pinhole or contamination pattern but rather a multitude of scratches, sporadic pinholes at the $1 \mu \mathrm{m}$ level, 'tracking', and other roughness features. Some of these inconsistencies are thought to result from die contamination, damage from rolling/post-processing, and/or lack of adhesion in the case of the metallized film layer. Representative micrographs for each film layer are shown in Figures 22-25. Micrographs $1 \mathrm{a}$ and $1 \mathrm{~b}$, Figure 22, are taken from the same sample area, but at different magnifications. The folds in these micrographs were found throughout all film layers. Samples 2 (Figure 22), 14 (Figure 24), and 16 \& 19 (Figure25) show the scratches and tracking patterns noted in all of the samples. It is not clear from these micrographs if the cause of some of the roughness is due to silica particles pushing their way through the layers. Many of the features that appear to be contamination or inconsistencies in the films were found in the metallized as well as the unmetallized film layers. The micrographs of the metallized film layer (Figure 25) raise questions as to the quality of the aluminum coating. Lack of a quality coating means permeation properties of the film are lost. Work will need to continue to develop an aluminum coating for PLA films of acceptable coat weight (optical density) and continuity. 
Figure 22. Non-Metallized Film, 4060D Corona-Treated Layer (458-72-2)
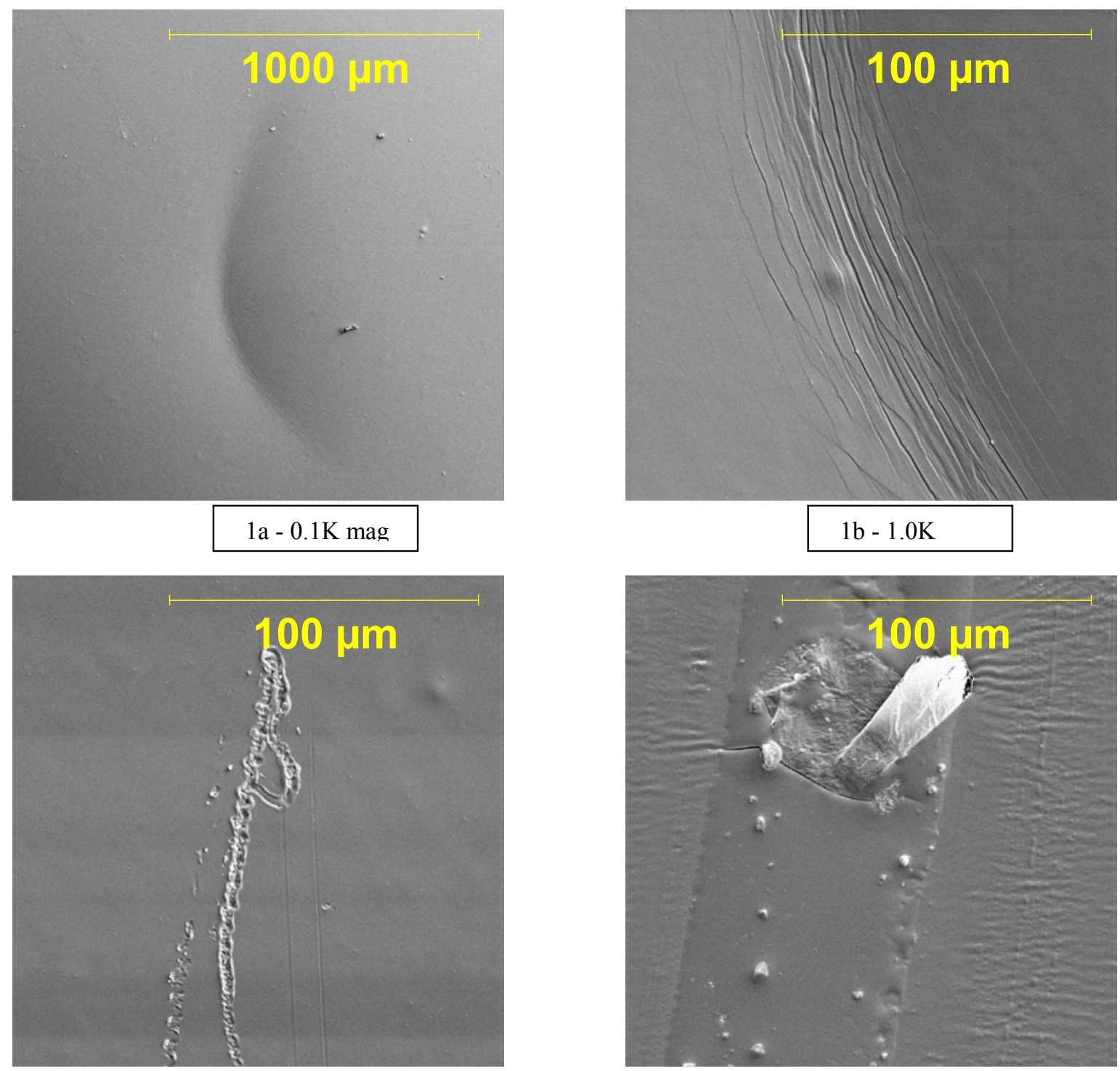

$$
2-1.0 \mathrm{~K}
$$

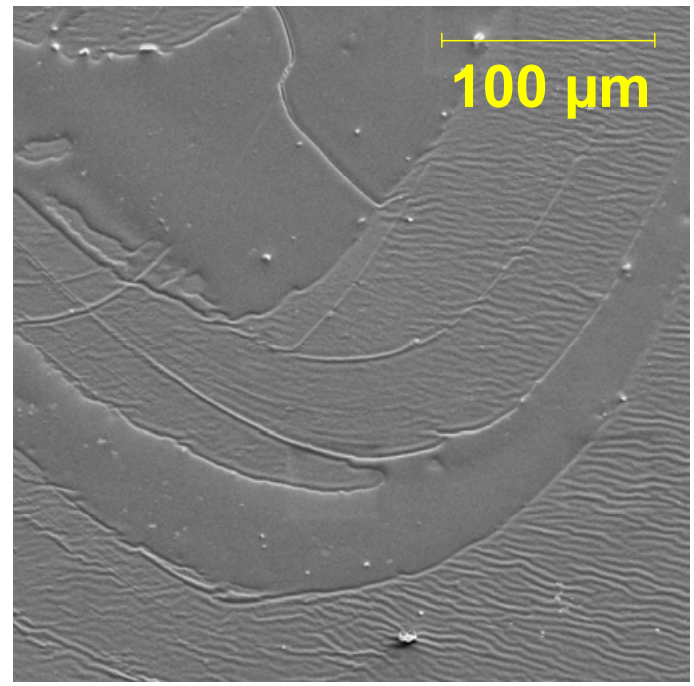

$4-1.0 \mathrm{~K}$ 
Figure 23. Non-Metallized Film, 4031D Fumed Silica Base Layer (458-72-2)
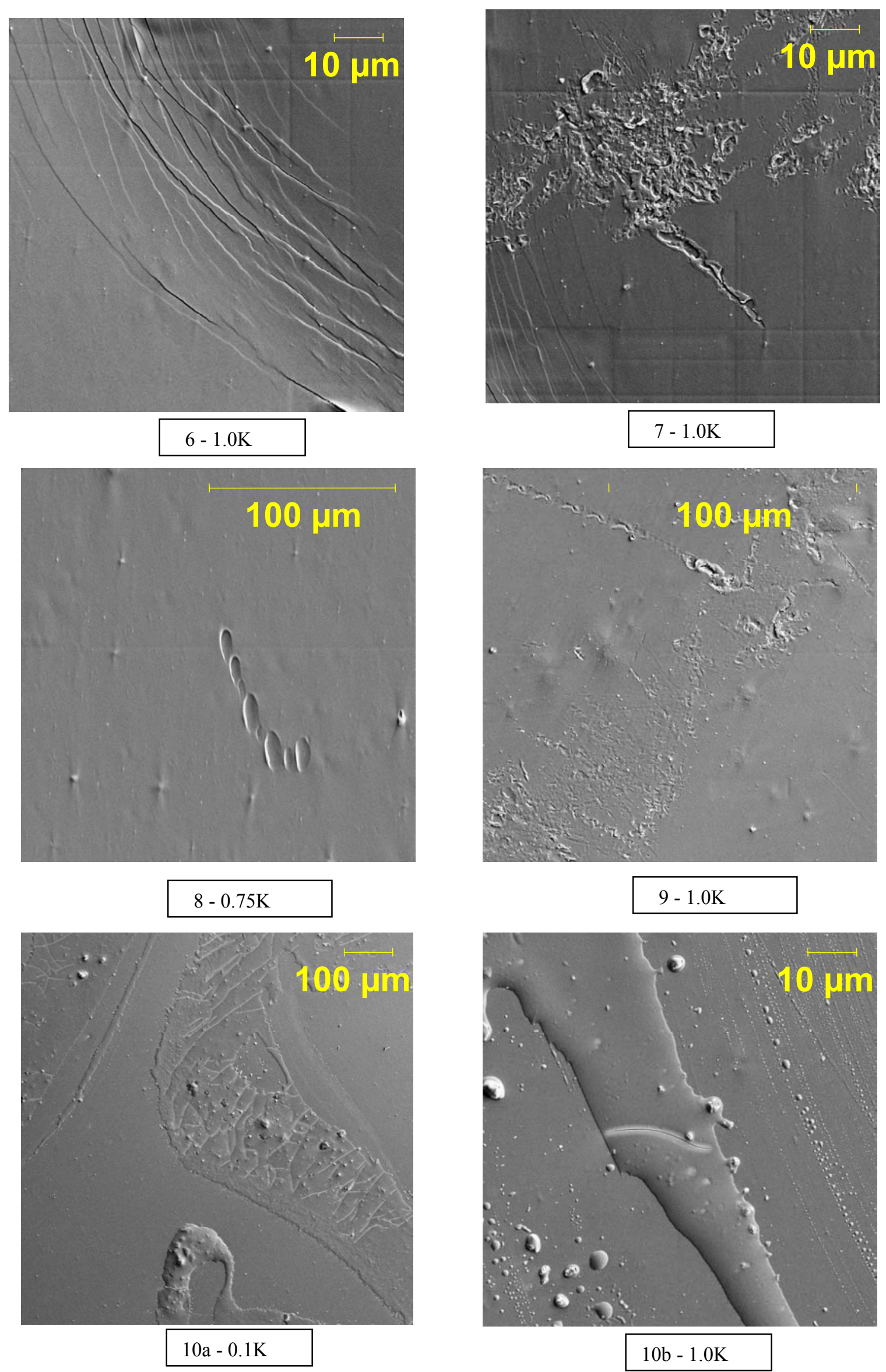
Figure 24. Metallized Film, 4031D Fumed Silica Base Layer (458-72-1)

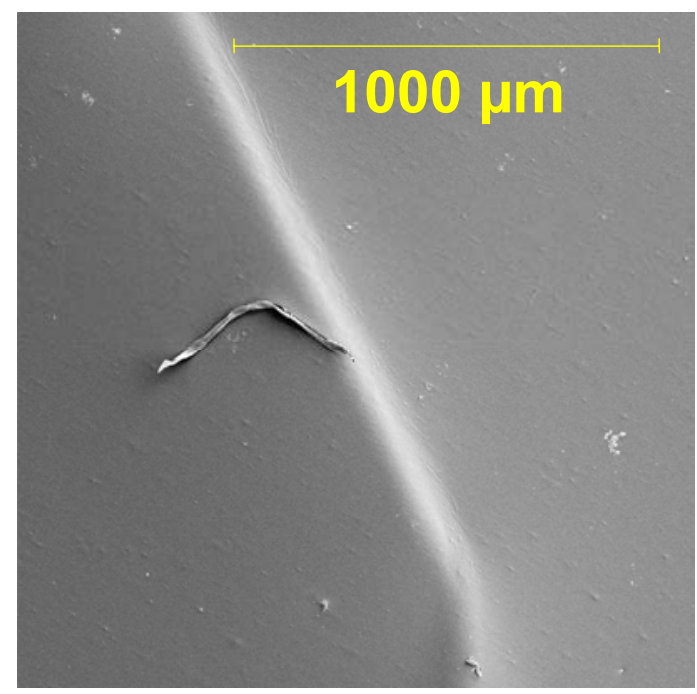

$11 \mathrm{a}-0.1 \mathrm{~K}$

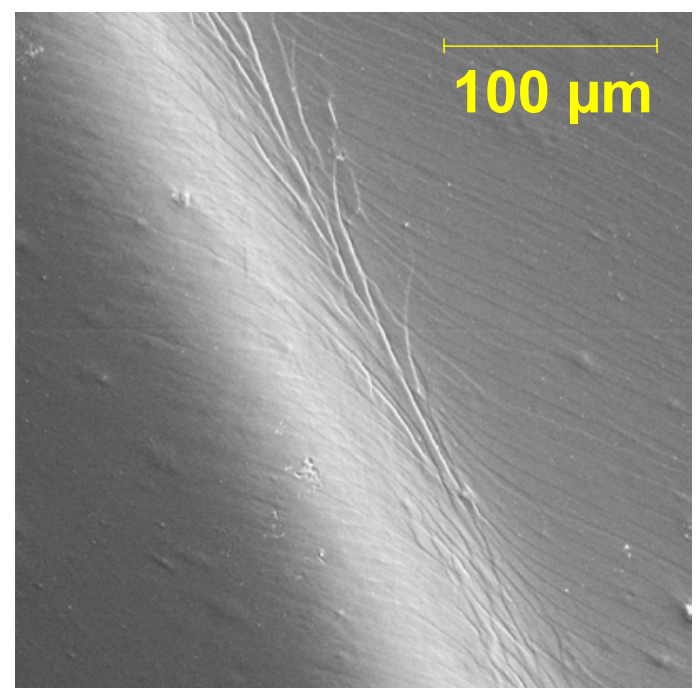

$11 \mathrm{~b}-0,5 \mathrm{~K}$

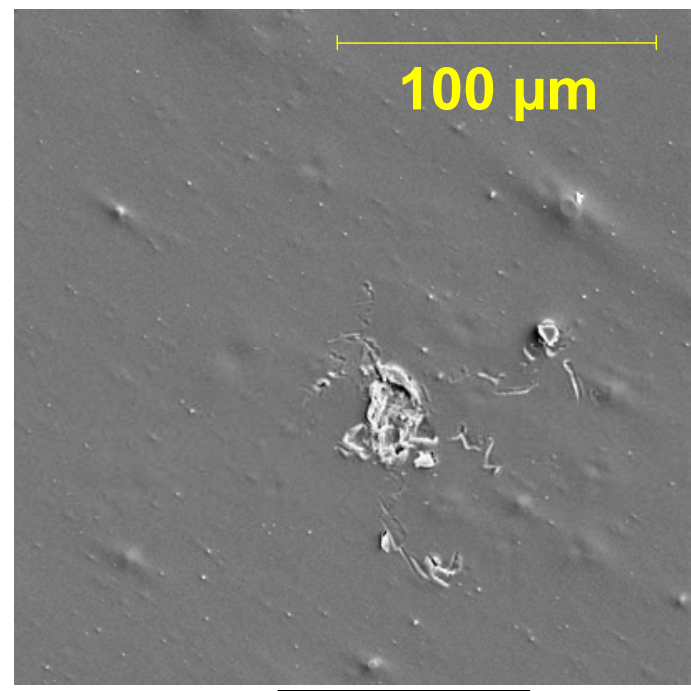

$12-0.75 \mathrm{~K}$

$13-1.0 \mathrm{~K}$

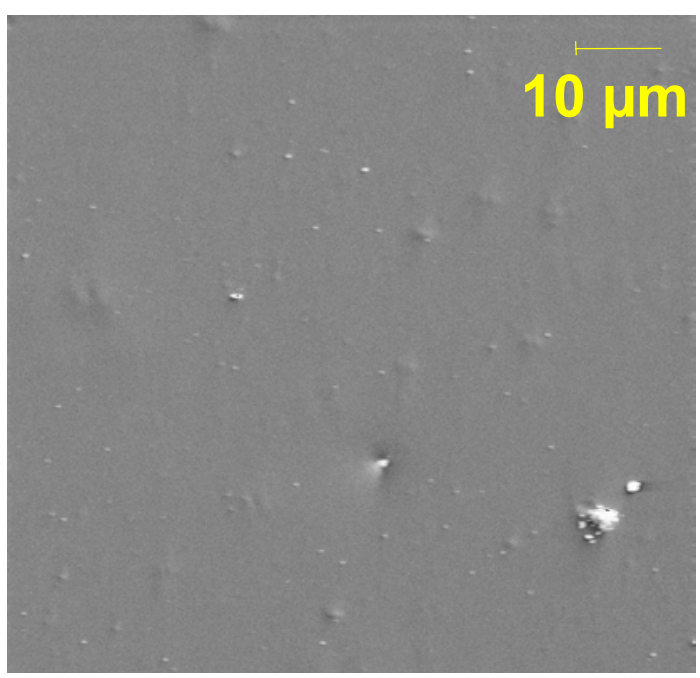

$14-1.0 \mathrm{~K}$ 
Figure 25. Metallized Film, 4060D Corona-Treated, Metallized side (458-72-1)
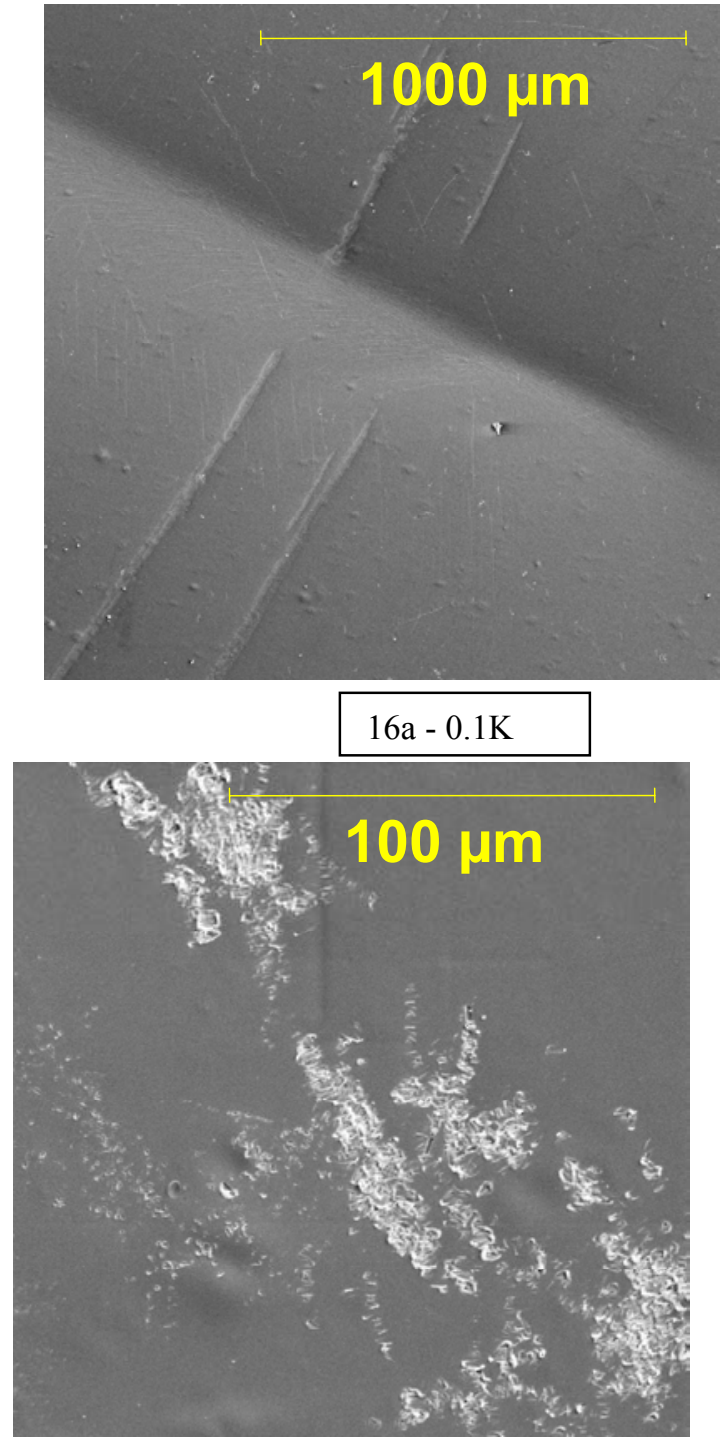

$17-1.0 \mathrm{~K}$

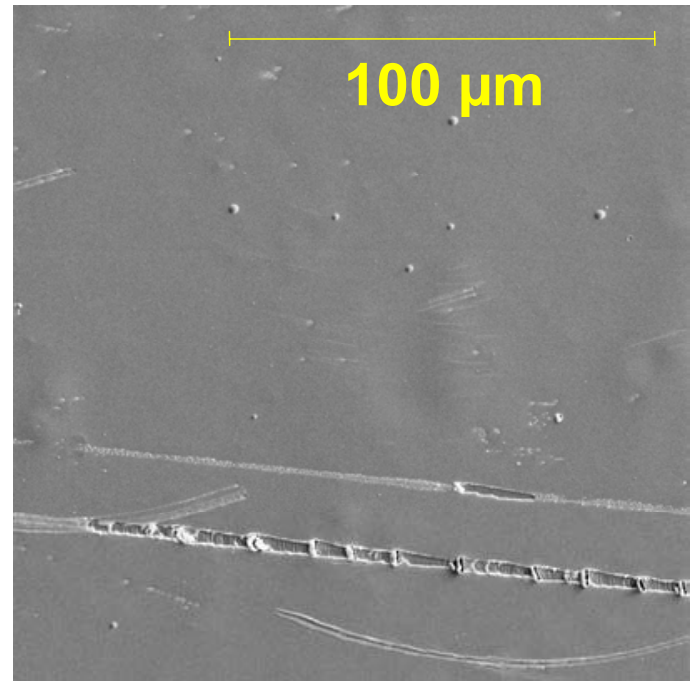

$19-1.0 \mathrm{~K}$
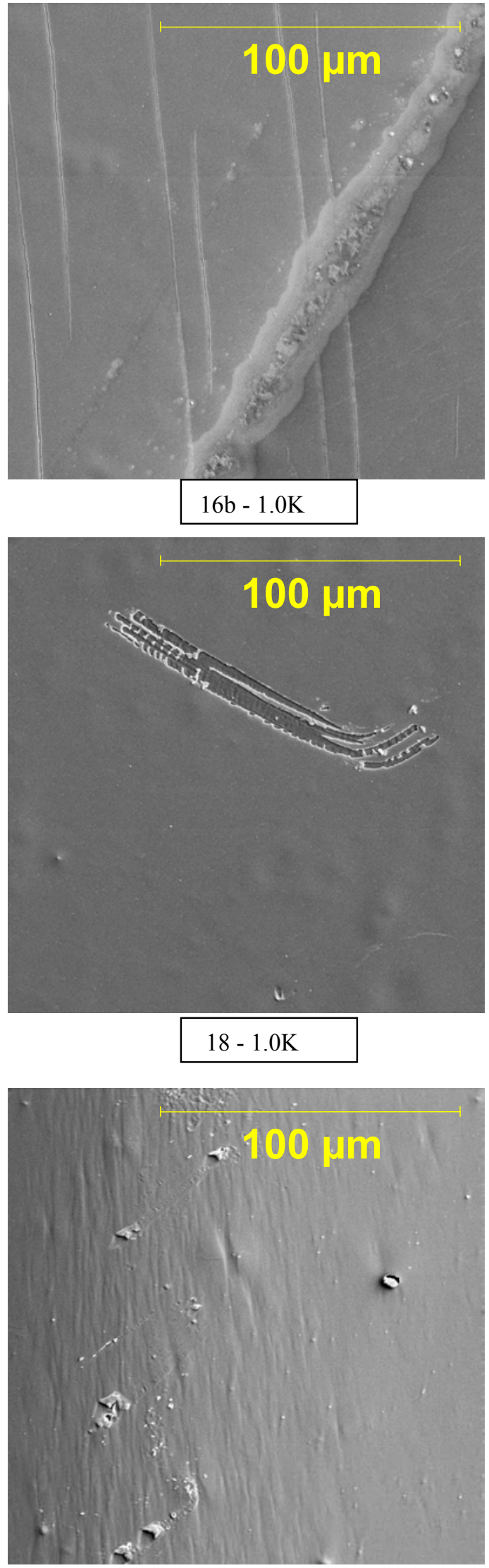

$20-1.0 \mathrm{~K}$ 


\subsection{Molecular Architecture and Phase Morphology}

\subsubsection{Synthesis and Characterization of Homopolymers}

\subsubsection{Controlled optical compositions and molecular weights for studies}

A wide range of optical compositions and molecular weights were required for the studies performed. This effort was led by Dan Knauss at the Colorado School of Mines.

\subsubsection{Kinetics of optical comonomer additions}

The rates of addition of various optical copolymer combinations were studied to determine the resulting sequence distrubutions. Solution H-NMR was used to characterize polymers throughout the course of polymerization. The studies utilize and contain Cargill Dow confidential information. Contact Jed Randall at 952-742-0453 or jed randall@cargilldow.com for more information.

\subsubsection{Synthesis and Characterization of Stereocomplexes}

A stereocomplex can form between chains or long segments of L-lactide rich PLA (PLLA) and D-rich PLA (PDLA) resulting in a crystal form with a significantly higher melting point than either homopolymer has on its own. This increase is approximately $+50^{\circ} \mathrm{C}$, and can result in much higher use temperatures compared to PLA homopolymer. Due to a lack of quantity of D-lactide, the quantity of material available for studies was limited. The studies utilize and contain Cargill Dow confidential information. Contact Jed Randall at 952-742-0453 or jed randall@cargilldow.com for more information.

\subsubsection{Synthesis of Copolymers}

De-emphasized as outlined in section 2 .

\subsubsection{Evaluation of branched copolymers}

Branched copolymers prepared by Colorado School of Mines and Cargill Dow were characterized for flow characteristics. The results appear in the following journal articles:

Melt Rheology of Poly(lactic acid): Consequences of Blending Chain Architectures $-\mathrm{H}$.J. Lehermeier, and J.R. Dorgan Polymer Engineering and Science, 2001, 41, No. 12, 2172-2184. (See abstract under section 3.1.2.2).

Thermal and rheological properties of commercial-grade poly(lactic acid)s. Dorgan, John R.; Lehermeier, Hans; Mang, Michael. Department of Chemical Engineering, Colorado School of Mines, Golden, CO, USA. Journal of Polymers and the Environment (2000), 8(1), $1-9$. 


\begin{abstract}
The thermal and rheol. properties of two com. grade poly(lactic acids) (PLA) were investigated. A comparison of the com. samples to a series of well-defined linear and star architecture PLA provides considerable insight into their flow properties. Such insights are valuable in deciding processing strategies for these newly emerging, com. significant, biodegradable plastics. Both a branched and linear grade PLA were investigated. The crystn. kinetics of the branched polymer are inferred to be faster than the linear analog. Longer relaxation times in the terminal region for the branched material compared to the linear material manifests itself as a higher zero shear rate viscosity. However, the branched material shear thins more strongly, resulting in a lower value of viscosity at high shear rates. Comparison of the linear viscoelastic spectra of the branched material with the spectra for star PLA suggests that the branched architecture is characterized by a span mol. wt. of .apprx. $63,000 \mathrm{~g} / \mathrm{mol}$. We demonstrate that a wide spectrum of flow properties are available through simple architectural modification of PLA, thus allowing the utilization of this important degradable thermoplastic in a variety of processing operations.

Additional branched copolymers, prepared by Cargill Dow, were characterized by Colorado School of Mines for flow and melt strength characteristics. The studies utilize and contain Cargill Dow confidential information. Contact Jed Randall at 952-742-0453 or jed randall@cargilldow.com for more information.
\end{abstract}

\title{
3.3.4. Formation of Clay Composites
}

De-emphasized within the context of the grant. See section 2 above. External program coordination was handled as part of Cargill Dow's cost share.

\subsubsection{Measurement of Permeation Properties}

3.3.5.1. Solubility and diffusivity contributions to permanent gas permeation.

A considerable effort was spent this quarter in finalizing the permeability measurements through PLA. The results are summarized in Tables 4 and 5; Table 4 gives the reference values at $30^{\circ} \mathrm{C}$ while Table 5 presents the activation energies. This information allows the calculation of permeability for any temperature below the glass transition temperature. Individual solubility and diffusivity contributions to the overall permeability were determined using a time lag apparatus. Values for the diffusivity determined by the time lag are given in column 4 of Table 4 and show the expected trend with molecular size (carbon dioxide has the smallest diffusivity, then oxygen, and nitrogen has the biggest diffusivity). Additional solubility measurements were made using a quartz crystal microbalance (QCM) and are presented in column 8 of Table 4 . These values are closer to the ideal values determined solely based on the vapor pressure of the respective gases. However, if the measured permeability is divided by the QCM solubility to get a diffusivity, $D_{\mathrm{QCM}}$, the values do not follow the proper order with molecular size of the gases. The same is true if the ideal solubility is used to determine a $D_{\text {ideal. }}$. It is concluded that the low permeability of nitrogen and oxygen are due to low solubility values compared to other polymeric materials but similar to other polyesters. 
Table 4. Permeability Values for permanent gases in PLA

\begin{tabular}{|c|c|c|c|c|c|c|c|c|}
\hline Permeant & L/D Ratio & $\begin{array}{c}\mathrm{P}_{\theta} \\
\left(30^{\circ} \mathrm{C}\right)\end{array}$ & $\begin{array}{c}\mathrm{D}_{\theta} \\
\left(30^{\circ} \mathrm{C}\right)\end{array}$ & $\begin{array}{c}\mathrm{D}_{\mathrm{QCM}} \\
\left(30^{\circ} \mathrm{C}\right)\end{array}$ & $\mathrm{D}_{\text {ideal }}$ & $\begin{array}{c}\mathrm{S}_{\theta} \\
\left(30^{\circ} \mathrm{C}\right)\end{array}$ & $\begin{array}{c}\mathrm{S}_{\mathrm{QCCM}} \\
\left(30^{\circ} \mathrm{C}\right)\end{array}$ & $\mathrm{S}_{\text {ideal }}$ \\
\hline $\mathrm{CO}_{2}$ & $98.7 / 1.3$ & 1.10 & $4.4 \mathrm{E}-09$ & & & 0.025 & & \\
& $80 / 20$ & 0.51 & $3.8 \mathrm{E}-09$ & $1.3 \mathrm{E}-09$ & $1.7 \mathrm{E}-09$ & 0.013 & 0.038 & 0.0295 \\
& $50 / 50$ & 0.71 & $4.5 \mathrm{E}-09$ & & & 0.016 & & \\
& & & & & & & & \\
$\mathrm{O}_{2}$ & $98.7 / 1.3$ & 0.26 & $5.7 \mathrm{E}-08$ & & & $4.9 \mathrm{E}-04$ & & \\
& $80 / 20$ & 0.18 & $5.6 \mathrm{E}-08$ & $1.5 \mathrm{E}-09$ & $3.3 \mathrm{E}-09$ & $3.2 \mathrm{E}-04$ & 0.012 & 0.0048 \\
& $50 / 50$ & 0.17 & $7.6 \mathrm{E}-08$ & & & $2.2 \mathrm{E}-04$ & & \\
& & & & & & & & \\
$\mathrm{~N}_{2}$ & $98.7 / 1.3$ & 0.05 & $2.4 \mathrm{E}-08$ & & & $2.2 \mathrm{E}-04$ & & \\
& $80 / 20$ & 0.02 & ${ }^{* * *}$ & $4.4 \mathrm{E}-10$ & $7.7 \mathrm{E}-10$ & ${ }^{* * *}$ & 0.005 & 0.0026 \\
& $50 / 50$ & 0.03 & ${ }^{* * *}$ & & & & & \\
& & & & & & \\
& & & & & & & \\
\end{tabular}

Units: P --- Barrer; D --- $\mathrm{cm}^{2} / \mathrm{s} ; \mathrm{S}$--- $\mathrm{cm}^{3} / \mathrm{cm}^{3}$ (polymer) $\mathrm{cmHg}$

Activation energies for permeability, diffusivity and solubility were all determined and are included in Table 5. It is to be noted that the permeabilities were found to be lower than in previous work. Also, all of the samples were solution cast and are largely amorphous thus a further conclusion of the work is that the effect of optical composition on amorphous phase permeability is small and probably negligible. Accordingly, permeability should be dominated by achievable crystallinity.

Table 5. Activation Energies for permeability of gases in PLAs.

Units: $\mathrm{EP}$, ED and $\mathrm{ES}$ in $\mathrm{KJ} / \mathrm{mol}$

\begin{tabular}{|c|c|c|c|c|}
\hline Permeant & L/D Ratio & $\mathrm{E}_{\mathrm{P}}$ & $\mathrm{E}_{\mathrm{D}}$ & $\mathrm{E}_{\mathrm{S}}$ \\
\hline $\mathrm{CO}_{2}$ & $98.7 / 1.3$ & 18.1 & 36.3 & -18.4 \\
& $80 / 20$ & 17.8 & 32.2 & -13.9 \\
& $50 / 50$ & 14.3 & 34.8 & -25.4 \\
& & & & \\
$\mathrm{O}_{2}$ & $98.7 / 1.3$ & 24.0 & 42.7 & -19.2 \\
& $80 / 20$ & 24.9 & 40.8 & -15.9 \\
& $50 / 50$ & 26.6 & 68.8 & -42.0 \\
& & & & \\
$\mathrm{~N}_{2}$ & $98.7 / 1.3$ & 34.6 & 59.3 & -25.0 \\
& $80 / 20$ & 40.9 & $* * *$ & $* * *$ \\
& $50 / 50$ & 35.0 & $* * *$ & $* * *$ \\
\hline
\end{tabular}

In addition, carbon dioxide permeability was determined for a promising blend system of PLA with Eastman Chemicals BioStar GP. The effects of a special flow additive developed under a different project were also investigated and found to be insignificant. The results are presented 
in Table 6 and were generated using "wide specification" PLA in the blends.

Table 6. Permeation properties of PLA:Biostar blends.

\begin{tabular}{|c|c|c|c|c|c|c|}
\hline Polymer & $\mathrm{P}\left(30^{\circ} \mathrm{C}\right)$ & $\mathrm{D}\left(30^{\circ} \mathrm{C}\right)$ & $\mathrm{S}\left(30^{\circ} \mathrm{C}\right)$ & $E_{P}$ & $E_{D}$ & $E_{s}$ \\
\hline $\begin{array}{l}\text { Eastar BioStar } \\
\text { GP (EB) }\end{array}$ & $14.6 \pm 0.6$ & $1.0 \mathrm{E}-7$ & 0.0143 & 34.5 & 35.5 & -1.7 \\
\hline $\begin{array}{l}\text { BASF EcoFlex } \\
(\mathrm{EF})\end{array}$ & $12.3 \pm 0.5$ & 8.1E-8 & 0.0148 & 35.3 & 44.6 & -9.7 \\
\hline PLA:EB $(90: 10)$ & $1.3 \pm 0.1$ & 5.3E-9 & 0.0243 & 18.6 & 37.3 & -18.8 \\
\hline $\begin{array}{c}\text { PLA:EB:EF } \\
(87: 10: 3)\end{array}$ & $1.2 \pm 0.1$ & $6.3 E-9$ & 0.0205 & 27.7 & 38.7 & -11.6 \\
\hline $\begin{array}{l}\text { PLA:EB:EF } \\
(89: 10: 1)\end{array}$ & $1.4 \pm 0.1$ & $6.5 \mathrm{E}-9$ & 0.0217 & 21.6 & 40.2 & -17.3 \\
\hline
\end{tabular}

\subsubsection{Comparison of steady-state gas permeation derived values to time lag measurements for permanent gases.}

The work conducted in this subtask has been or will be published in the journal articles listed below.

Gas Permeation Properties of Poly(lactic acid) - H.J. Lehermeier, J.R. Dorgan, and J.D. Way, Journal of Membrane Science, 2001, 190, 243-251.

\section{Abstract}

This study examines the permeation of nitrogen, oxygen, carbon dioxide, and methane in amorphous films of PLA cast from solution. The properties of PLA are compared to other commodity plastics and it is shown that PLA permeation closely resembles that of polystyrene. At $30{ }^{\circ} \mathrm{C}, \mathrm{N}_{2}$ permeation in PLA is $1.3\left(10^{-10} \mathrm{~cm}^{3}(\mathrm{STP}) \cdot \mathrm{cm} / \mathrm{cm}^{2} \cdot \mathrm{s} \cdot \mathrm{cm} \mathrm{Hg}\right)$ and the activation energy is $11.2 \mathrm{~kJ} / \mathrm{mole}$. For oxygen the corresponding values are $3.3\left(10^{-10}\right.$

$\mathrm{cm}^{3}(\mathrm{STP}) \cdot \mathrm{cm} / \mathrm{cm}^{2} \cdot \mathrm{s} \cdot \mathrm{cm} \mathrm{Hg}$ ) and $11.1 \mathrm{~kJ} / \mathrm{mol}$. The values for carbon dioxide permeation are 1.2 $\left(10^{-10} \mathrm{~cm}^{3}(\mathrm{STP}) \cdot \mathrm{cm} / \mathrm{cm}^{2} \cdot \mathrm{s} \cdot \mathrm{cm} \mathrm{Hg}\right)$ and $6.1 \mathrm{~kJ} / \mathrm{mol}$. For methane values of $1.0\left(10^{-10}\right.$

$\mathrm{cm}^{3}(\mathrm{STP}) \cdot \mathrm{cm} / \mathrm{cm}^{2} \cdot \mathrm{s} \cdot \mathrm{cm} \mathrm{Hg}$ ) and an activation energy of $13.0 \mathrm{~kJ} / \mathrm{mol}$ are found. Studies with pure gases show that polymer chain branching and small changes in L:D stereochemical content have no effect on permeation properties. Crystallinity is found to dominate permeation properties in a biaxially oriented film. The separation factor for a $\mathrm{CO}_{2} / \mathrm{CH}_{4}$ mixed gas system is measured between 0 and $50{ }^{\circ} \mathrm{C}$ and does not deviate significantly from the calculated ideal separation factor; at $0{ }^{\circ} \mathrm{C}$ the separation factor is 16 , a value that suggests continued studies of PLA as a separation medium are warranted.

Gas Permeation Properties of Poly(lactic acid) Revisited. Lihong Bao, John R. Dorgan, J. Douglas Way, Dan Knauss, Sukendu Hait, submitted to Journal of Membrane Science, 2003

\footnotetext{
Abstract

Pure gas permeation through solution-cast amorphous poly(lactic acid) (PLA) films is investigated using the time-lag method. New data on permeability, diffusivity and solubility for $\mathrm{N}_{2}, \mathrm{O}_{2}$ and $\mathrm{CO}_{2}$ are reported. At $30^{\circ} \mathrm{C}, \mathrm{N}_{2}$ permeability, diffusivity and solubility in PLA having a stereochemical L:D composition of $98.7: 1.3 \%$ are 0.05 Barrer, $2.4 \mathrm{E}-8 \mathrm{~cm}^{2} / \mathrm{s}$, and $2.2 \mathrm{E}-4$ $\mathrm{cm}^{3}(\mathrm{STP}) / \mathrm{cm}^{3}$ (polymer) $\mathrm{cmHg}$ and the activation energy of permeation is $34.6 \mathrm{~kJ} / \mathrm{mol}$. For $\mathrm{O}_{2}$,
} 
the corresponding values are 0.26 Barrer, $5.7 \mathrm{E}-8 \mathrm{~cm}^{2} / \mathrm{s}, 4.9 \mathrm{E}-4 \mathrm{~cm}^{3}(\mathrm{STP}) / \mathrm{cm}^{3}$ (polymer) $\mathrm{cmHg}$ and $24.0 \mathrm{~kJ} / \mathrm{mol}$. The values for $\mathrm{CO}_{2}$ are 1.10 Barrer, $4.4 \mathrm{E}-9 \mathrm{~cm}^{2} / \mathrm{s}, 0.025$

$\mathrm{cm}^{3}(\mathrm{STP}) / \mathrm{cm}^{3}$ (polymer) $\mathrm{cmHg}$ and $18.0 \mathrm{~kJ} / \mathrm{mol}$. These new measurements of the gas permeation properties of poly(lactic acid) (PLA) show considerable disagreement with previously reported results (J.M.S., 190 (2001) 243-251) obtained using a continuous flow apparatus. The effect of PLA optical compositions on permeation is also investigated and found to be consistent with the earlier report; the L/D ratio has no effect on the permeation properties provided the material is quenched into an amorphous state. The ability to generate diffusivity and solubility data along with the greater sensitivity of pressure measurement over gas composition argue in favor of the time-lag permeability measurements and suggest that the revised PLA permeation values reported here should be adopted.

\section{Final Milestone Log}

\begin{tabular}{|l|l|l|l|l|}
\hline $\begin{array}{l}\text { ID } \\
\text { Number }\end{array}$ & Task/Milestone & $\begin{array}{l}\text { Planned } \\
\text { Completi } \\
\text { on }\end{array}$ & $\begin{array}{l}\text { Actual } \\
\text { Complet } \\
\text { ion }\end{array}$ & Comments \\
\hline 1.1 & Characteristic Ratios & $9 / 02$ & $1 / 03$ & Completed \\
\hline 1.2 & Measurements of Radii of Gyrations & $6 / 03$ & $6 / 02$ & Completed \\
\hline 1.3 & Preparation of PLA blends & $6 / 03$ & $12 / 01$ & Completed \\
\hline 1.4 & Linear Viscoelastic Properties & $6 / 03$ & $1 / 01$ & Completed \\
\hline 1.5 & Extensional Viscosity Properties & $6 / 03$ & $1 / 01$ & Completed \\
\hline 1.6 & Steady shear viscosity measurements & $6 / 03$ & $1 / 01$ & Completed \\
\hline 1.7 & Crystallization studies & $6 / 03$ & $6 / 03$ & Completed \\
\hline 1.8 & Processing studies & $6 / 03$ & $6 / 03$ & Completed \\
\hline 1.9 & Development of a predictive model & $6 / 03$ & $6 / 03$ & $\begin{array}{l}\text { Partially } \\
\text { Completed }\end{array}$ \\
\hline 2.1 & Preparation of Model Biomass Surfaces & $6 / 03$ & $2 / 01$ & Completed \\
\hline 2.2 & Preparation of Model Metal Surfaces & $6 / 03$ & $6 / 03$ & Completed \\
\hline 2.3 & Xray Measurements of PLA Interdiffusion & $6 / 03$ & & De-emphasized \\
\hline 2.4 & Ellipsometric Measurements of PLA & $6 / 03$ & $12 / 02$ & Completed \\
\hline 2.5 & Molecular Modeling of PLA & $6 / 03$ & $6 / 03$ & Completed \\
\hline 3.1 & $\begin{array}{l}\text { Synthesis and Characterization of } \\
\text { Homopolymers }\end{array}$ & $6 / 03$ & $4 / 02$ & Completed \\
\hline 3.2 & $\begin{array}{l}\text { Synthesis and Characterization of } \\
\text { Stereocomplexes }\end{array}$ & $6 / 03$ & $6 / 03$ & Completed \\
\hline 3.3 & Sythesis of copolymers & $6 / 03$ & $1 / 01$ & Complete \\
\hline 3.4 & Formation of Clay Composites & $6 / 03$ & $9 / 02$ & Complete \\
\hline
\end{tabular}





\section{Intellectual Property Summary}

1. Title: Method for Producing Semi-Crystalline Polylactic Acid Articles

Inventors: Richard C. Bopp; Jason Whelan

Publication of the Invention: None.

Patent Status: This Subject Invention was described in United States provisional application 60,313,685, filed August 20, 2001, PCT application, filed August 19, 2002 (and designating all PCT member states and regional organizations except the US), and US non-provisional application 10/223,451, filed August 19, 2002.

\section{Budget Summary}

\subsection{Final accounting of DOE and Cargill Dow contributions}

\begin{tabular}{|l|r|r|r|r|r|r|r|r|}
\hline \multicolumn{2}{|l|}{ Phase / Budget Period } & \multicolumn{2}{|l|}{ Approved Spending Plan } & \multicolumn{2}{l|}{ Actual Spent to Date } \\
\hline & From & To & DOE Amount & Cost Share & Total & DOE Amount & Cost Share & Total \\
\hline Year 1 & $10 / 1 / 1999$ & $9 / 30 / 2000$ & 198794 & 353079 & 551873 & 198794 & 353079 & 551873 \\
\hline Year 2 & $10 / 1 / 2000$ & $9 / 30 / 2001$ & 371463 & 263900 & 635363 & 371463 & 263900 & 635363 \\
\hline Year 3 & $10 / 1 / 2001$ & $9 / 30 / 2002$ & 433542 & 466669 & 900211 & 433542 & 466669 & 900211 \\
\hline Year 4 & $10 / 1 / 2002$ & $6 / 30 / 2003$ & 179886 & 253261 & 433147 & 179886 & 222818 & 402704 \\
\hline Year 5 & & & & & & & & \\
\hline & & & & & & & & \\
\hline Totals & & & 1183685 & 1336909 & 2520594 & 1183685 & 1306466 & 2490151 \\
\hline
\end{tabular}

\begin{tabular}{|c|c|c|c|}
\hline & DOE Amount & Cost Share & Total \\
\hline Remaining funds & 0 & 30443 & 30443 \\
\hline
\end{tabular}

\section{Attachments}

\section{Publication submissions}

Melt Rheology of Variable L-content Poly(lactic acid). Janzen, Jay, Dorgan, John R., Clayton, Michael P., Knauss, Daniel M., and Hait, Sukhendu B. submitted to Macromolecules, 2003.

Gas Permeation Properties of Poly(lactic acid) Revisited. Bao, Lihong, Dorgan, John R., Way, J. Douglas, Knauss, Dan, Hait, Sukendu, submitted to Journal of Membrane Science, 2003.

Fundamental Solution and Single-Chain Properties of Polylactides. Dorgan, John R., Janzen, Jay, Knauss, Daniel M., Hait, Sukhendu B., Limoges, Bradford R., 
and Hutchinson, Matthew H. submitted to Macromolecules 2003

Blends of Poly(lactic acid) with a Degradable Aliphatic-Aromatic Polyester Tercopolymer, Bao, Lihong, Dorgan, John D., and Muzzy, John. submitted to Polymer Science and

Engineering 2003

\section{Publications}

Melt Rheology of Poly(lactic acid): Consequences of Blending Chain Architectures - H.J. Lehermeier, and J.R. Dorgan, Polymer Engineering and Science,2001, 41, No. 12, 2172-2184.

Local and global dynamics of polylactides. Mierzwa, M.; Floudas, G.; Dorgan, J.; Knauss, D.; Wegner, J. Journal of Noncrystalline Solids, 2002, vol. 307-310, pp. 296-303

A novel approach to modeling viscoelastic properties of thermoplastic polymer melts, with applications to polylactides. Janzen, Jay; Dorgan, John R.. Colorado School of Mines, Golden, CO, USA. Annual Technical Conference - Society of Plastics Engineers, 2002, 60th(Vol. 1), 929-931

Polylactides: rheology and processing of a commercial polymer from renewable resources. Dorgan, John R.; Janzen, Jay; Cicero, John. Chem. Eng. Dept., Colorado School of Mines, Golden, CO, USA. Polymeric Materials Science and Engineering , 2002, 86 348-349

Melt Rheology of High L-content Poly(lactic acid) - I.L. Palade, H.J. Lehermeier, and J.R. Dorgan, Macromolecules, 2001, 34, 1384- 1390.

Effects of molecular architecture on two-step, melt-spun poly(lactic acid) fibers. Cicero, John A.; Dorgan, John R.; Garrett, James; Runt, James; Lin, J. S. Department of Chemical Engineering, Colorado School of Mines, Golden, CO, USA. Journal of Applied Polymer Science, 2002, 86(11), 2839-2846.

Supramolecular morphology of two-step, melt-spun poly(lactic acid) fibers. Cicero, John A.; Dorgan, John R.; Janzen, Jay; Garrett, James; Runt, James; Lin, J. S. Department of Chemical Engineering, Colorado School of Mines, Golden, CO, USA. Journal of Applied Polymer Science , 2002, 86(11), 2828-2838.

Phosphite stabilization effects on two-step melt-spun fibers of polylactide. Cicero, John A.; Dorgan, John R.; Dec, Steven F.; Knauss, Daniel M. Department of Chemical Engineering, Colorado School of Mines, Golden, CO, USA. Polymer Degradation and Stability, 2002, 78(1), 95-105.

Physical Properties and Fiber Morphology of Poly(lactic acid) Obtained from Continuous Two-Step Melt Spinning. C Cicero, John A.; Dorgan, John R.. Department of Chemical Engineering, Colorado School of Mines, Golden, CO, USA. Journal of Polymers and the Environment, 2001, Volume 9(1), 1-15. 
Gas Permeation Properties of Poly(lactic acid), H.J. Lehermeier, J.R. Dorgan, and J.D. Way, Journal of Membrane Science 190, pp. 243-251 (2001).

Polylactides: Properties and Prospects of an Environmentally Benign Plastic from Renewable Resources, Dorgan, J. R.; Lehermeier, H. J.; Palade, L.-I.; Cicero, J., Macromolecular Symposia, 2001, vol. 175, pp. 55-66

Thermal and Rheological Properties of Commercial Grade Poly(lactic acids), Dorgan,J.R. ; Lehermeier, H.J. and Mang M. , Journal of Polymers and the Environment, 2000, 8(1), 1-9. 


\section{Key Contributors}

\section{Cargill Dow LLC}

Laura Babcock

Richard C. Bopp

Wendy Christensen

Kevin Cink

Robert Kean

Michael Mang

Kevin McCarthy

Eric Meierdierks

Jed Randall

\section{Colorado School of Mines}

Lihong Bao

John Dorgan

Jay Janzen

Sukendu Hait

Dan Knauss

National Renewable Energy Laboratory (NREL)

Steve Kelley

Heidi Pilath 


\title{
Blends of Poly(lactic acid) with a Degradable Aliphatic-Aromatic Polyester Tercopolymer
}

\author{
Lihong Bao $^{\mathrm{a}}$, John D. Dorgan ${ }^{\mathrm{a}}$ and John Muzzy ${ }^{\mathrm{b}}$ \\ ${ }^{a}$ Chemical Engineering Department, Colorado School of Mines, Golden, CO 80401 \\ ${ }^{\mathrm{b}}$ Chemical Engineering Department, Georgia Institute of Technology, Atlanta, GA 30332
}

\section{INTRODUCTION}

Polymers from renewable resources is a growing paradigm in the polymers industry. Recent developments have led to an expanding range of potential applications, especially in packing and fibers. Polylactides (PLAs) have been known for several decades and have recently gained considerable commercial significance [1]. PLA is currently the most produced biobased polymer derived from agriculture resources through bioconversion and polymerization. Large scale of commercial production has already begun.

Extensive research work related to PLA synthesis, chemical, physicochemical and mechanical properties has been performed [2]. Most of its properties are comparable to those of synthetic polymer from fossil-oil such as Polyethylene (PE), Polystyrene (PS), Polypropylene (PP) and Polyethylene terephthalate (PET). Figure 1 shows the comparison of mechanical properties among these polymers. It can be seen that PLA is much like Polystyrene, it has relatively high modulus but is brittle. Another potential problem with PLA is that it has reasonably high polarity that can cause adhesive problems with certain substrates leading to processing difficulties.

To improve mechanical properties and processibility of PLA and retain its biodegradability, blending of PLA with other biodegradable polymer is logical. Biodegradable polyesters generally work well in blends with PLA, starch, and natural-fiber reinforcements. A broad range 
of synthetic biodegradable resins based on aliphatic polyesters and aliphatic-aromatic copolyesters have been commercialized [3]. Eastar ${ }^{\circledR}$ Bio polyesters from Eastman Chemical Company are a family of aliphatic-aromatic copolyesters based on butanediol, adipic acid, and terephthalic acid. Eastar Bio GP (general purpose) has a melting point of $108^{\circ} \mathrm{C}$ and offers good contact clarity and adhesion along with high elongation to break (up to $800 \%$ ). This material also possesses high moisture and grease resistance while processing much like LDPE. Shortcomings for some applications include relatively low stiffness, poor melt strength, and a tendency to stick in injection molds. Such synthetic aliphatic-aromatic biodegradable polyesters (BASF makes a similar commercial product called EcoFlex) tend to complement the properties of PLA and are a logical choice for improving mechanical properties through blending while retaining environmental degradability.

The objective of this article is to investigate the properties of PLA/Eastar BioGP blends. Data on the melt rheological properties, mechanical properties, and gas $\left(\mathrm{CO}_{2}\right)$ permeation are presented.

\section{EXPERIMENTAL}

\section{Materials \& Methods}

PLA pellets were obtained from Cargill-Dow LLC (Minnetonka, Minnesota). The average molecular weight is $100,000 \mathrm{~g} / \mathrm{mol}$ and the optical composition is $\mathrm{L}: \mathrm{D}=98: 2$.

Eastar ${ }^{\circledR}$ Bio GP aliphatic-aromatic copolyester with density of $1.22 \mathrm{~g} / \mathrm{cm} 3$ and glass transition temperature of $-30{ }^{\circ} \mathrm{C}$ was obtained from Eastman Chemical Company (Kingsport, Tennessee).

Reagent grade methylene chloride that was $99.6 \%$ pure was purchased from Aldrich and used as received as the solvent for film casting. 
High purity gases were used to ensure accuracy of the data. $\mathrm{CO}_{2}$ was $99.99 \%$ pure obtained from General Air.

PLA and Eastar BioGP were blended in a Berstorff $25 \mathrm{~mm}$ co-rotating, intermeshing twinscrew extruder that operated at $250 \mathrm{rpm}$. The mixing zone consisted of both kneading blocks and pin mixing elements and was vacuum vented. The temperature profile had a maximum zone temperature of $165^{\circ} \mathrm{C}$. Materials were pre-mixed to the desired weight fractions in pellet form and fed to the extruder using a Ktron loss-in-weight feeder. Blends were quenched into a water bath and pelletized using a Killion knife blade type pelletizer. Susequently, samples were dried in a vacuum oven or by using desiccated air prior to further experimentation.

Miscibility of PLA and Eastar copolyester was studied using differential scanning calorimetry (Perkin-Elmer model DSC 7). It is well known that for miscible polymer blends, only one glass transition temperature $\left(\mathrm{T}_{\mathrm{g}}\right)$ exists, however, two $\mathrm{T}_{\mathrm{g}} \mathrm{s}$ should be observed for blends from two immiscible polymers. The films made for permeation testing were also observed using a Zeiss optical microscope.

Blends as well as pure polymers were tested using an ARES rheometer from Rheometric Scientific (Piscataway, NJ). Due to degradation effects, exposure times in the rheometer were minimized using an established protocol.

PLA and copolyester blends were compression-molded into tensile bars (type IV) following ASTM test method D-638-91 using a hot press (Carver Inc., Wabash, IN) at a temperature of 176 ${ }^{\circ} \mathrm{C}$ and molding pressure of 4.2 $\mathrm{MPa}$. Molded specimens were then cooled down before removal from the mold. Tensile strength and elongation at break were determined using an Instron testing system (Model 4465, Canton, MA) according to ASTM D-638-91 with a crosshead speed of 5 $\mathrm{mm} / \mathrm{min}$ and $25 \mathrm{~mm}$ gauge length. Samples were tested three to five times each. Other samples 
were injection molded prior to mechanical testing so that a comparison between molding methods was possible.

To prepare films for permeation testing, 8-10 wt.\% solutions of the compounded blends in methylene chloride were prepared and films were cast using a Gardner casting blade onto a glass plate. The glass plate was put into a fume hood for several hours to allow the solvent evaporate from the film. Films were subsequently floated off the glass in a water bath, recovered, and dried overnight in a vacuum oven at room temperature.

A time-lag apparatus for measuring pure gas permeation was used to test $\mathrm{CO}_{2}$ permeation properties in the blends as well as the constituent polymers. The permeation properties of $\mathrm{CO}_{2}$ and other gases $\left(\mathrm{N}_{2}\right.$ and $\left.\mathrm{O}_{2}\right)$ in PLA have been tested on the apparatus and are reported elsewhere [5]; the details of the presents tests were the same as in that report.

\section{Results and Discussions}

\section{Microstructure}

The macroscopic properties of polymer blends are highly associated with the microstructure of the materials. Of particularly significant importance ins whether the blends for a single homogeneous thermodynamic phase or if they are biphasic with a major continuous phase and a dispersed minor phase. The later is the situation in the present blends. As mentioned above, when a blend is biphasic, two distinct glass transition temperatures are observed in a DSC experiment and this is the case for the present blends. In addition, the two phase nature of the blends was directly observed using polarizing optical microscopy. Figure 2a shows results for a 90:10 PLA:EastarBioGP blend while Figure 2b shows the micrograph for a 70:30 blend. In both cases there is a dispersed minor phase; in the 70:30 blend the dispersed phase droplet size is 
considerably larger. It is concluded that the morphology of the blends consists of a PLA major phase with a droplet dispersed BioGP minor phase.

Figure 3 shows a comparison of the dynamic viscosities of PLA, BioGP, and their blends (PLA:Eastar $=90: 10$ and 80:20) measured at $180{ }^{\circ} \mathrm{C}$. For all samples typical flow curves are observed with a shear thinning behavior being evident. Because of its higher glass transition temperature, PLA has a higher viscosity that the BioGP material; viscosity of the blends lies between these two limits. It is important to note that the addition of the BioGP reduces viscosity; this potentially provides a processing advantage of the blends over pure PLA in processing operations where pressure and flowability are important, for example, in injection molding applications.

Figures 4-6 show the modulus, impact strength and elongation of pure PLA and the blends with two different BioGP percentages. In Figure 3, modulus data from the compression molded samples produced at the Colorado School of Mines are compared to the injection molded samples prepared at the Georgia Institute of Technology. For pure PLA and the 90/10 PLA/BioGP blend, the two sets of results are in good agreement. Both results show that as the BioGP content increases, the blend modulus decreases. However, the impact strength is increased as illustrated in Figure 4. That is, blending PLA with the BioGP copolyester increases the material impact strength. The most significant advantage of blending is evident in Figure 6. The PLA blend with $20 \%$ copolyester shows a dramatic increase of about 40 times in the strain at break. The mechanical properties of PLA are greatly altered by blending with the copolyester; a much more ductile material can be made with only modest losses in the modulus.

For gas permeation in pure PLA, it was found that permeability was highly affected by the annealing of the membrane film [5]. For the PLA blends, similar effects were observed, even 
though the magnitude of effect depends on the PLA contents in the blends. For Eastar copolyester, annealing has almost no effect as it is above its glass transition temperature under use conditions. All the permeation results shown here are from films annealed at $45 \mathrm{C}$. Also, only $\mathrm{CO}_{2}$ permeation in the blend is measured as it serves as a probe to understand the effects of blending on permeation properties.

At $30{ }^{\circ} \mathrm{C}, \mathrm{CO}_{2}$ permeability in PLA is about 1.1 Barrers $\left(1\right.$ Barrer $=10^{-10} \mathrm{~cm}^{3}(\mathrm{STP}) \mathrm{cm} / \mathrm{cm}^{2} \mathrm{~s}$ $\mathrm{cmHg}$ ), for Eastar copolyester, the value is much greater - about 14.6 Barrers. That is, PLA being a glassy material at $30{ }^{\circ} \mathrm{C}$ allows far less gas transport than the BioGP which is subbery at this temperature. Accordingly, the blend permeabilities are determined by the Eastar copolyester content. The temperature dependence of $\mathrm{CO}_{2}$ permeability, diffusivity and solubility in the $90 / 10$ PLA/Eastar belnd is shown in Figures 7-9. The permeability of this blend is about 1.30 Barrers at $30^{\circ} \mathrm{C}$, only a little bit larger than that of PLA. However, as the Eastar copolyester content goes up, the blend permeability becomes larger. As the temperature increases, $\mathrm{CO}_{2}$ solubility decreases because $\mathrm{CO}_{2}$ is a permanent gas. The activation energy of solubility measures how strong an effect this is for a particular material and temperature has less effect on the $\mathrm{CO}_{2}$ solubility in the BioGP copolyester.

Similar results are found for the other blends having different compositions. Table 1 summarizes the test results for all samples. The activation energies of permeation, diffusion and solution are included in the table. The physical effect responsible for the much greater permeation in the BioGP versus the PLA is evident from these results. Namely the diffusivity of the gas in the rubbery BioGP is 22.7 times greater than its diffusivity in the glassy PLA.

The following empirical model has been used to describe the permeability values in both homogeneous and heterogeneous binary blends and copolymers [6]. 


$$
\log P_{\text {blend }}=\phi_{1} \log P_{1}+\phi_{2} \log P_{2}
$$

where $P_{\text {blend }}$ is the blend gas permeability, $\phi_{1}$ and $\phi_{2}$ are the volume fractions of polymers 1 and 2 in the blend, and $P_{1}$ and $P_{2}$ are the permeabilities of polymers 1 and 2. Figure 10 represents the experimental and theoretical blend permeabilities of PLA/Eastar copolyester blends. A positive deviation from Equation 1 with an increase in Eastar copolyester concentration is obvious and is sometimes interpreted as a reflection of the biphasic nature of a blend. By considering the binary interactions, a modified equation based on Equation 1 was given in the literature [7]. Further investigation to the theoretical desciption of gas permeation in the blends is needed, however, this is beyond the scope of the present investigation.

$\mathrm{O}_{2}$ permeation properties in the polymer materials are very important to packaging applications. Even through $\mathrm{CO}_{2}$ was used for the permeation test, $\mathrm{O}_{2}$ permeation properties are expected to have similar trends so that the log-additivity rule of Equation 1 should provide a reasonable estimate of the blend permeation properties.

\section{Conclusions}

Mechanical and permeation properties of biodegradable PLA and its blends with biodegradable Eastar copolyester were investigated. PLA blends with Eastar copolyester provide significant property improvements - viscosity is decreased while toughness is increased at the

cost of only a modest decrease in modulus. $\mathrm{CO}_{2}$ permeation properties in pure polymers and blends have been determined. Blends have higher gas permeabilities than pure PLA. A positive deviation of experimental results from theoretical prediction was observed. 


\section{Acknowledgements}

Funding was provided by the EPA/NSF Technology for a Sustainable Environment Program (No. R 826733-01-0) and by the Agriculture Industries of the Future Program of the Office of Industrial Technologies, U.S. Department of Energy.

\section{References}

[1] John R. Dorgan, Jay Janzen, Lihong Bao, "Polylactide fundamentals and property modification by blending", $11^{\text {th }}$ Annual meeting of the BioEnvironmental Polymer Society, Denver, CO, August 10-13, 2003.

[2] Donald Garlotta, “A literature review of Poly(lactic acid)', Journal of Polymers and the Environmental, Vol.9, No.2, 63-84 (April 2001).

[3] Robert Leaversuch, "Biodegradable Polyesters: Packaging goes green", Online article of www.plasticstechnology.com, 09/02/2002.

[4] Majid Tayeb, Lihong Bao and John D. Dorgan, "Design of a time-lag apparatus for measuring pure gas permeation properties in polymeric membranes", In Preparation, Review of Scientific Instruments, 2004.

[5] Lihong Bao and John D. Dorgan, "Permeation properties of Poly(lactic acid). Revisited", submitted to Journal of Membrane Science, September 2003.

[6] J. H. Peteropoulos, "A comparative study of approaches applied to the permeability of binary composite polymeric materials", Journal of Polymer Science: Polymer Physics edition, Vol.23, No.7, 1309-1324 (July 1985).

[7] R. J. Li, W. P. Hsu, T. K. Kwei, and A. S. Myerson, "Transport of gases in miscible polymer blends above and below the glass transition temperature", AIChE Journal, Vol.39, No.9, 1509-1518 (September 1993). 
Table 1. Summary of $\mathrm{CO}_{2}$ permeation in PLA/Eastar copolyester blends

\begin{tabular}{|c|c|c|c|c|c|c|}
\hline Sample & $\mathrm{P}\left(30{ }^{\circ} \mathrm{C}\right)$ & $\mathrm{D}\left(30{ }^{\circ} \mathrm{C}\right)$ & $\mathrm{S}\left(30{ }^{\circ} \mathrm{C}\right)$ & $\mathrm{E}_{\mathrm{P}}$ & $\mathrm{E}_{\mathrm{D}}$ & $\mathrm{E}_{\mathrm{S}}$ \\
\hline PLA & 1.1 & $4.4 \mathrm{E}-9$ & 0.025 & 18.1 & 36.3 & -18.4 \\
\hline Eastar copolyester & 14.6 & $1.0 \mathrm{E}-7$ & 0.014 & 34.5 & 35.5 & -1.7 \\
\hline PLA:Eastar $=90: 10$ & 1.3 & $5.3 \mathrm{E}-9$ & 0.024 & 18.6 & 37.3 & -18.8 \\
\hline PLA:Eastar $=70: 30$ & 3.3 & $2.0 \mathrm{E}-8$ & 0.016 & 30.7 & 37.9 & -7.2 \\
\hline PLA $:$ Eastar $=60: 40$ & 4.4 & $1.7 \mathrm{E}-8$ & 0.024 & 29.4 & 52.5 & -23.3 \\
\hline PLA:Eastar $=30: 70$ & 7.7 & $4.3 \mathrm{E}-8$ & 0.018 & 37.7 & 53.1 & -15.5 \\
\hline PLA:Eastar $=20: 80$ & 12.3 & $7.3 \mathrm{E}-8$ & 0.017 & 35.5 & 55.7 & -18.9 \\
\hline
\end{tabular}


Figure Captions:

Figure 1. Comparison of mechanical properties of PLA and other common polymers.

Figure 2. Microscope Picture of PLA/Eastar blends. (a) PLA:Eastar = 90:10;

(b) PLA:Eastar $=70: 30$.

Figure 3. Dynamic viscosity of PLA, Eastar copolyester and their blends.

Figure 4. Modulus of PLA and PLA blends. Data from CSM and GIT are shown in the same plot for comparison.

Figure 5. Impact Strength of PLA and PLA blend. Blend composition is PLA:Eastar $=90: 10$.

Figure 6. Comparison of strain at break of PLA and its blends.

Figure 7.Temperature dependence of PLA blend (PLA:Eastar = 90:10) permeability.

Figure 8. Temperature dependence of PLA blend (PLA:Eastar $=90: 10)$ diffusivity.

Figure 9. Temperature dependence of PLA blend (PLA:Eastar = 90:10) solubility

Figure 10. Experimental and theoretical blend permeabilities of PLA/Eastar copolyester. 


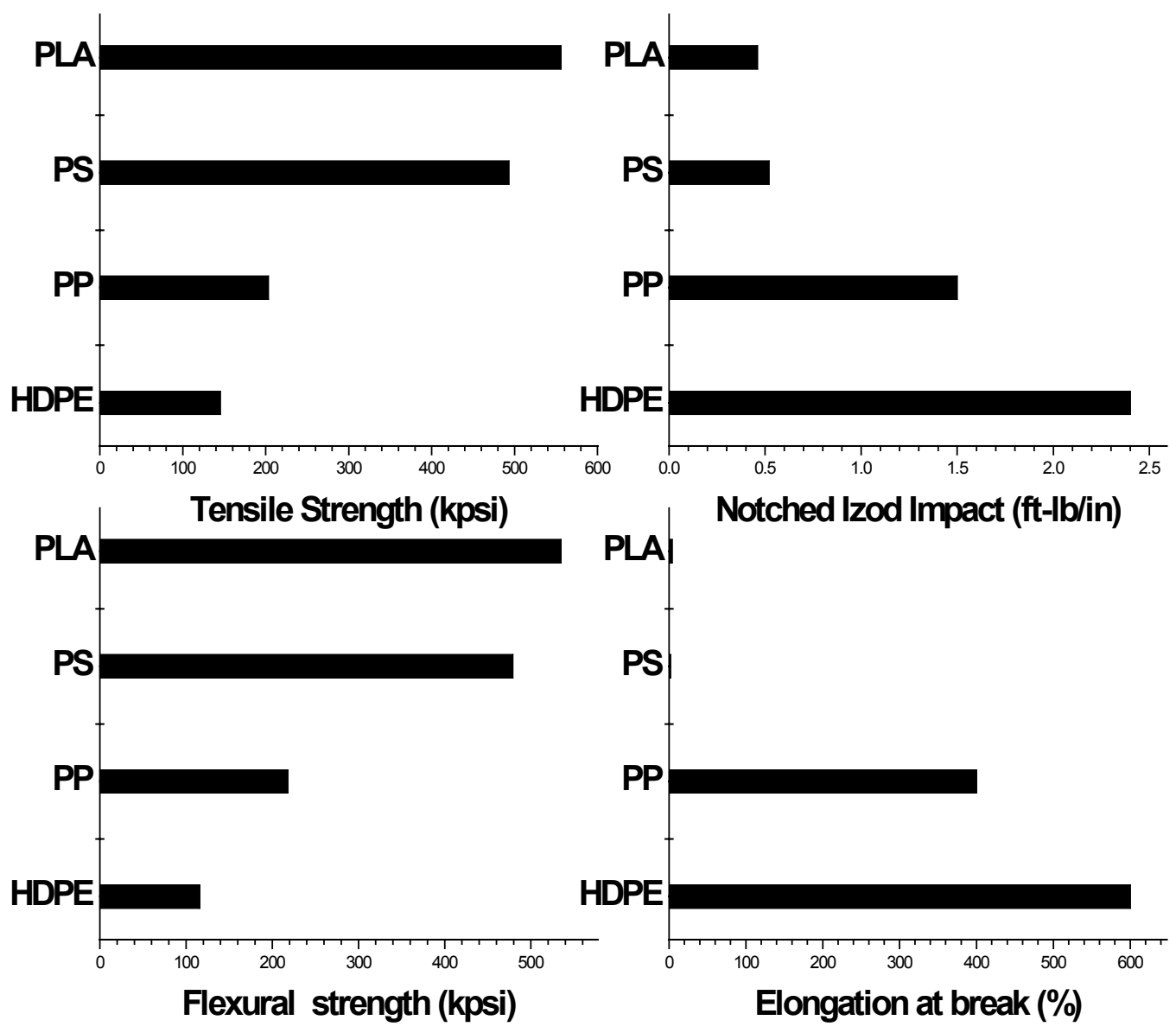

Bao and Dorgan Fig 1 
(a)

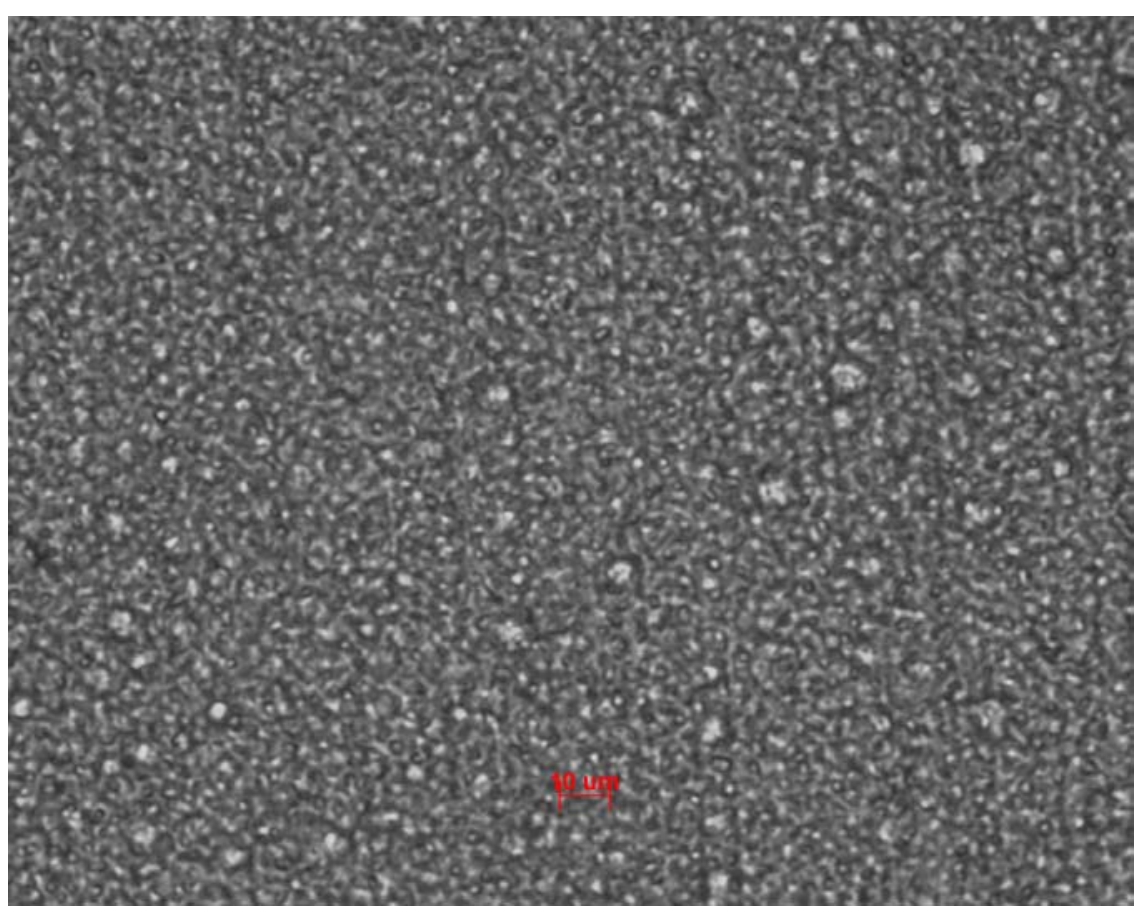

(b)

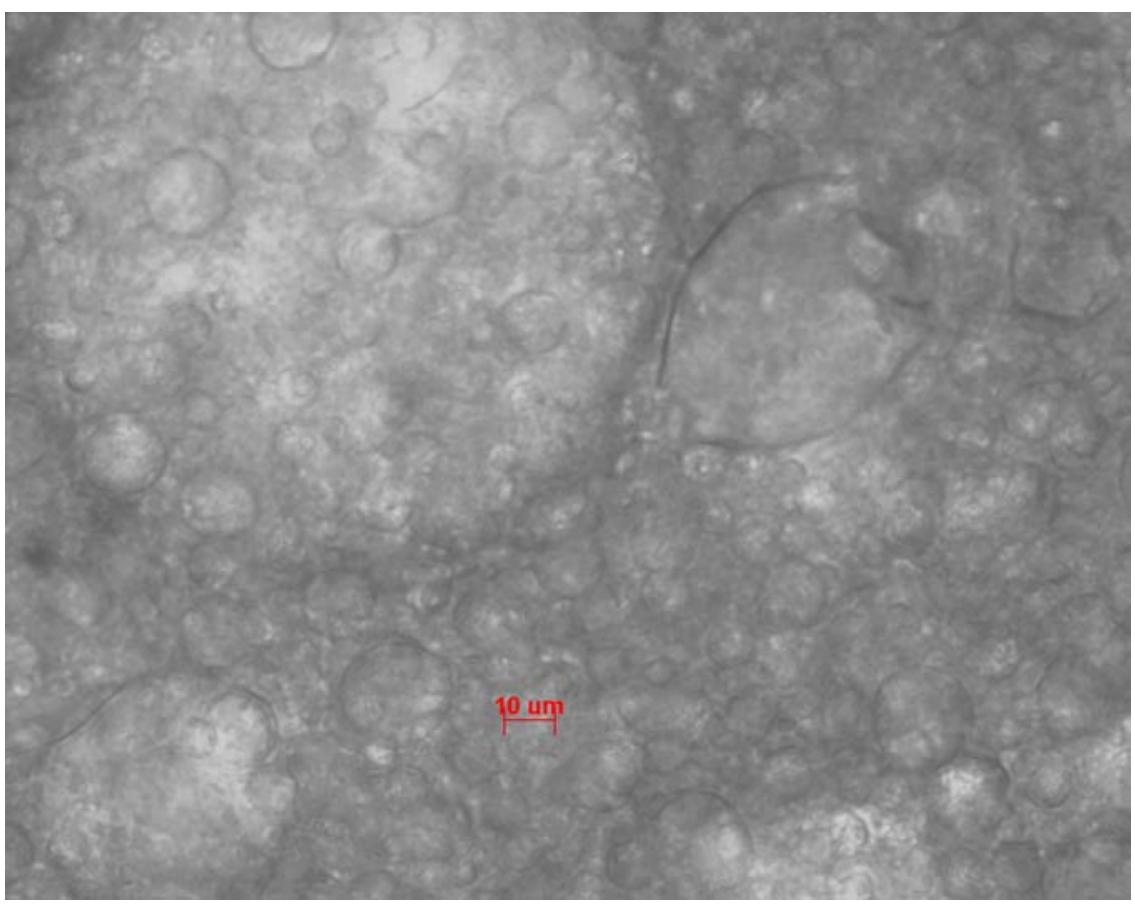

Bao and Dorgan Fig 2 


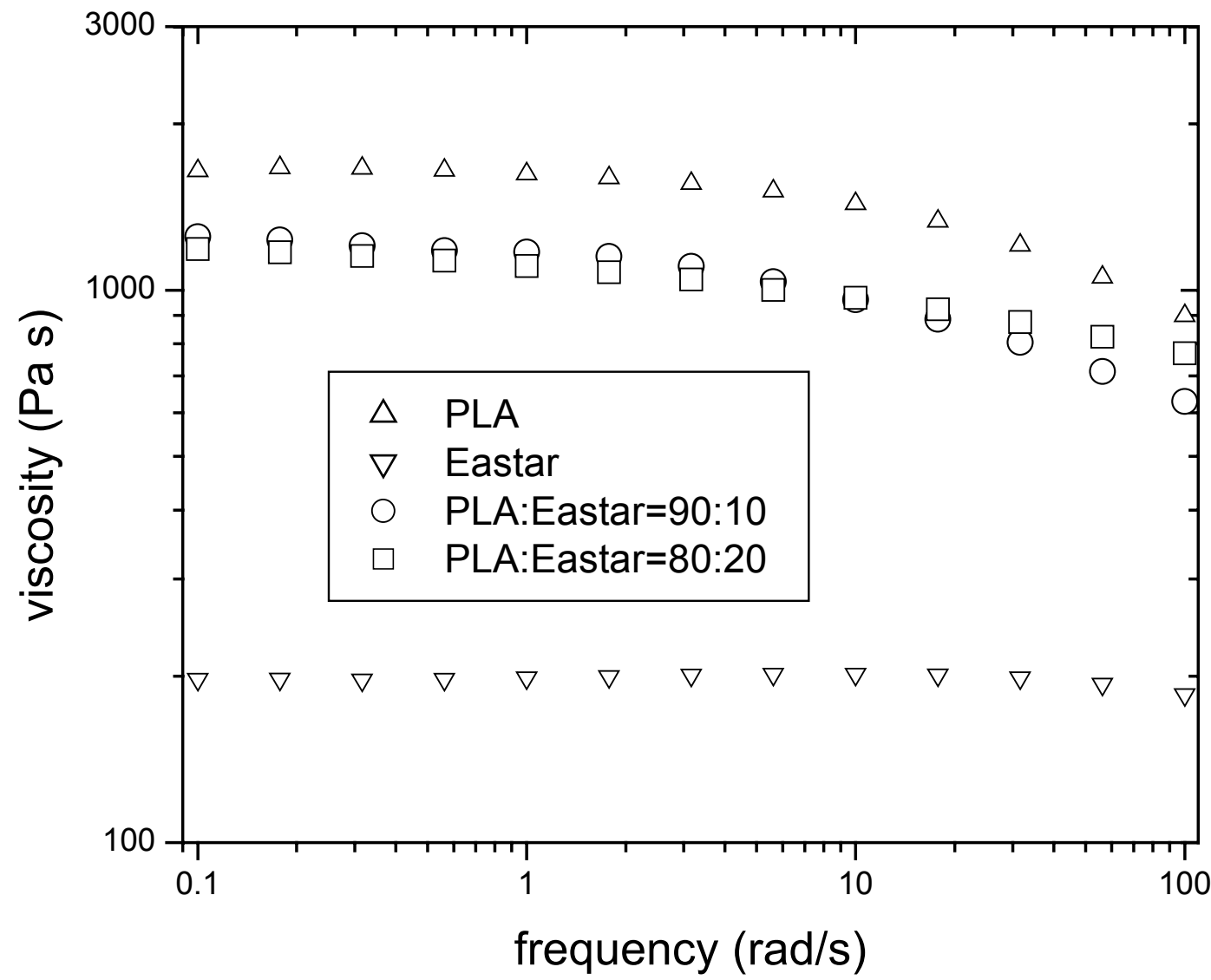


Bao \& Dorgan Fig3

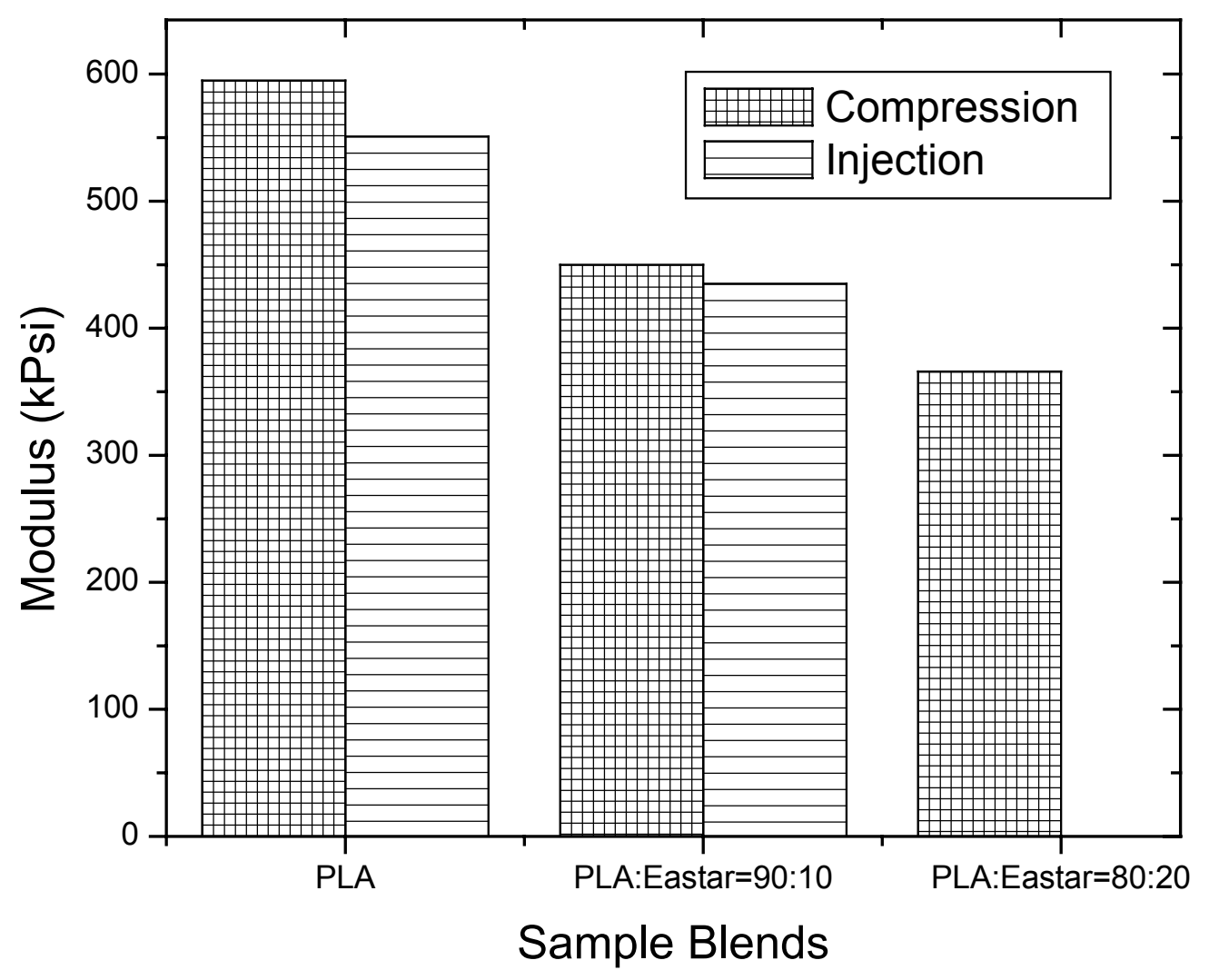


Bao and Dorgan Fig 4

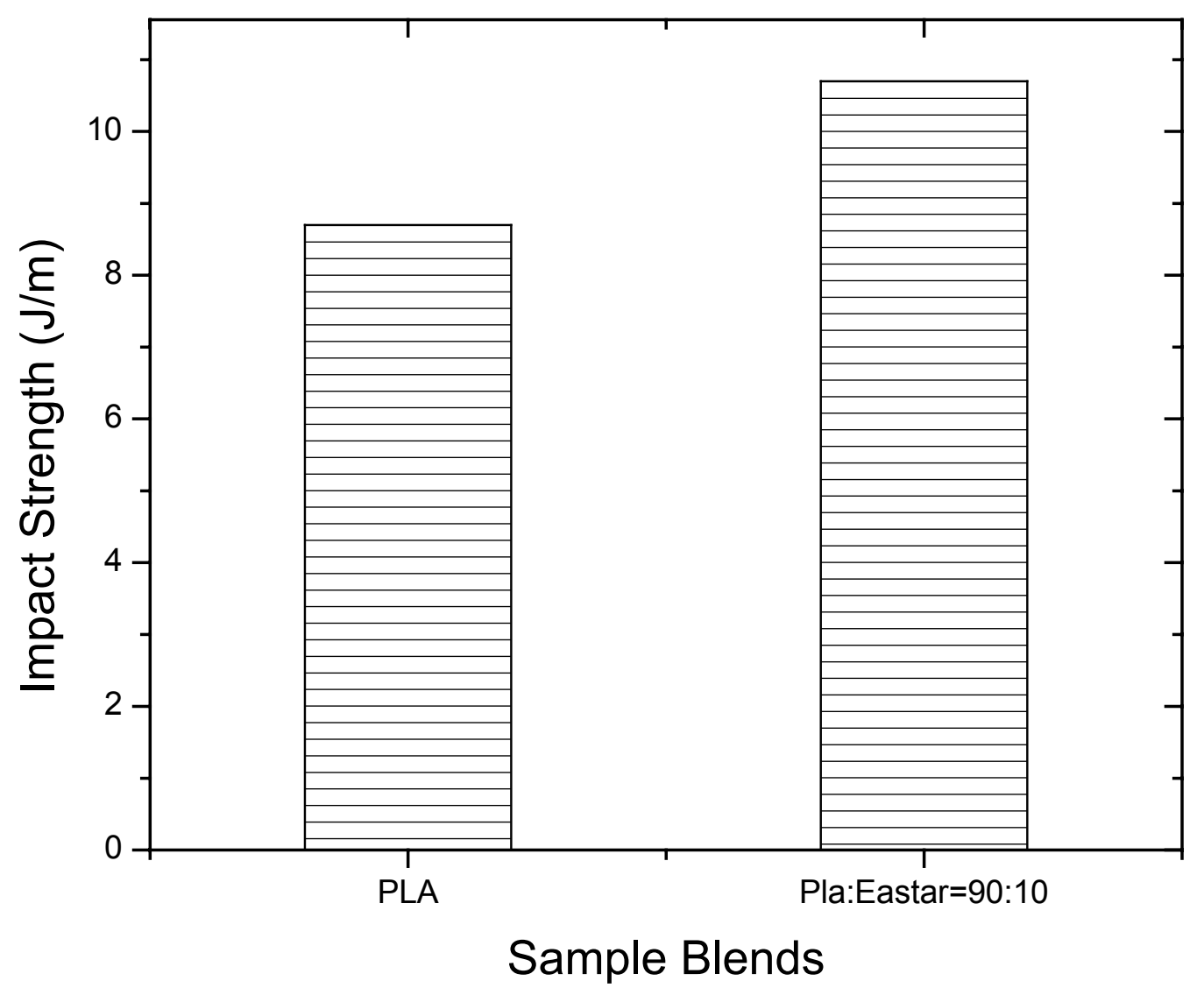


Bao and Dorgan Fig 5

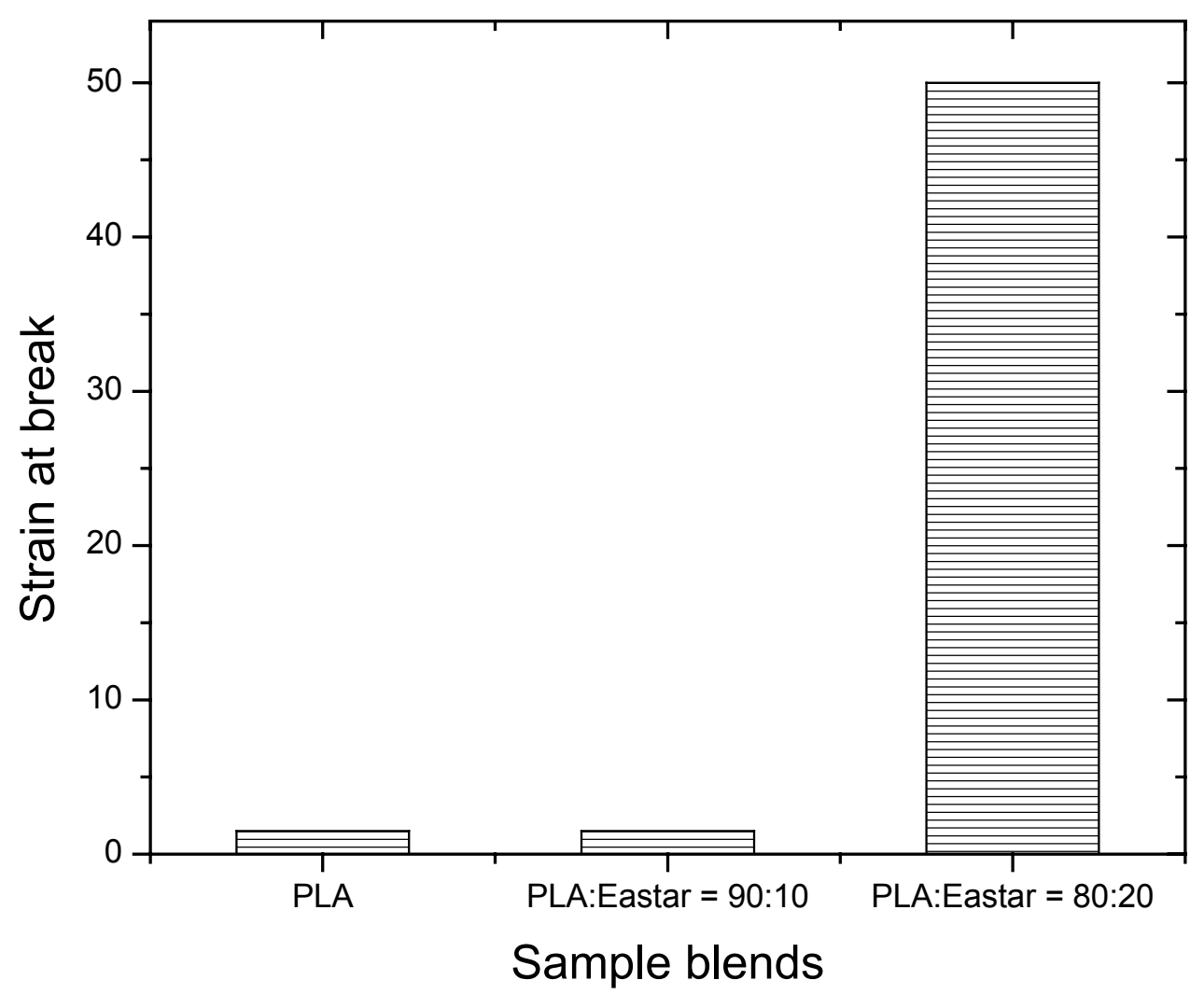


Bao and Dorgan Fig 6

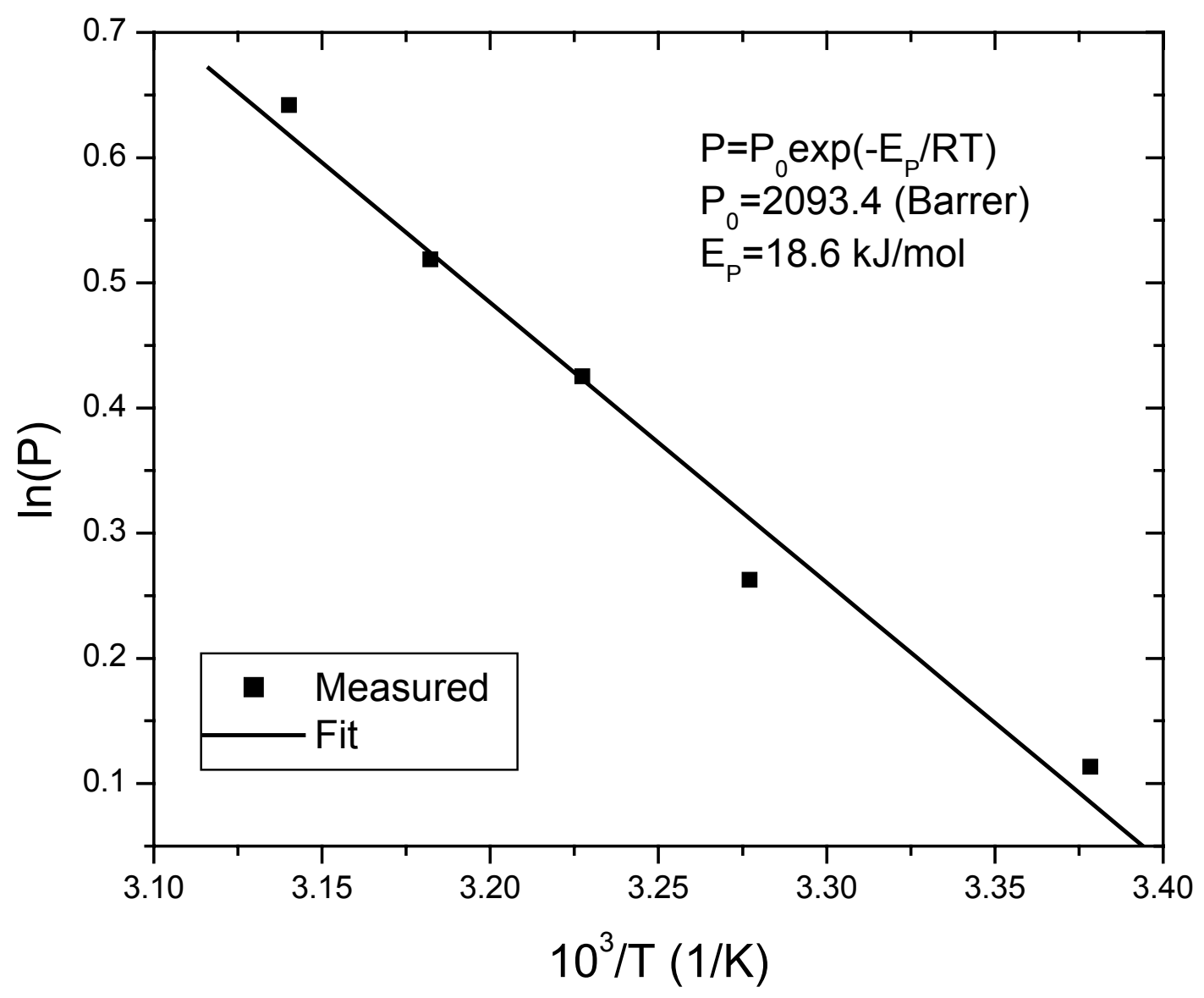


Bao and Dorgan Fig 7

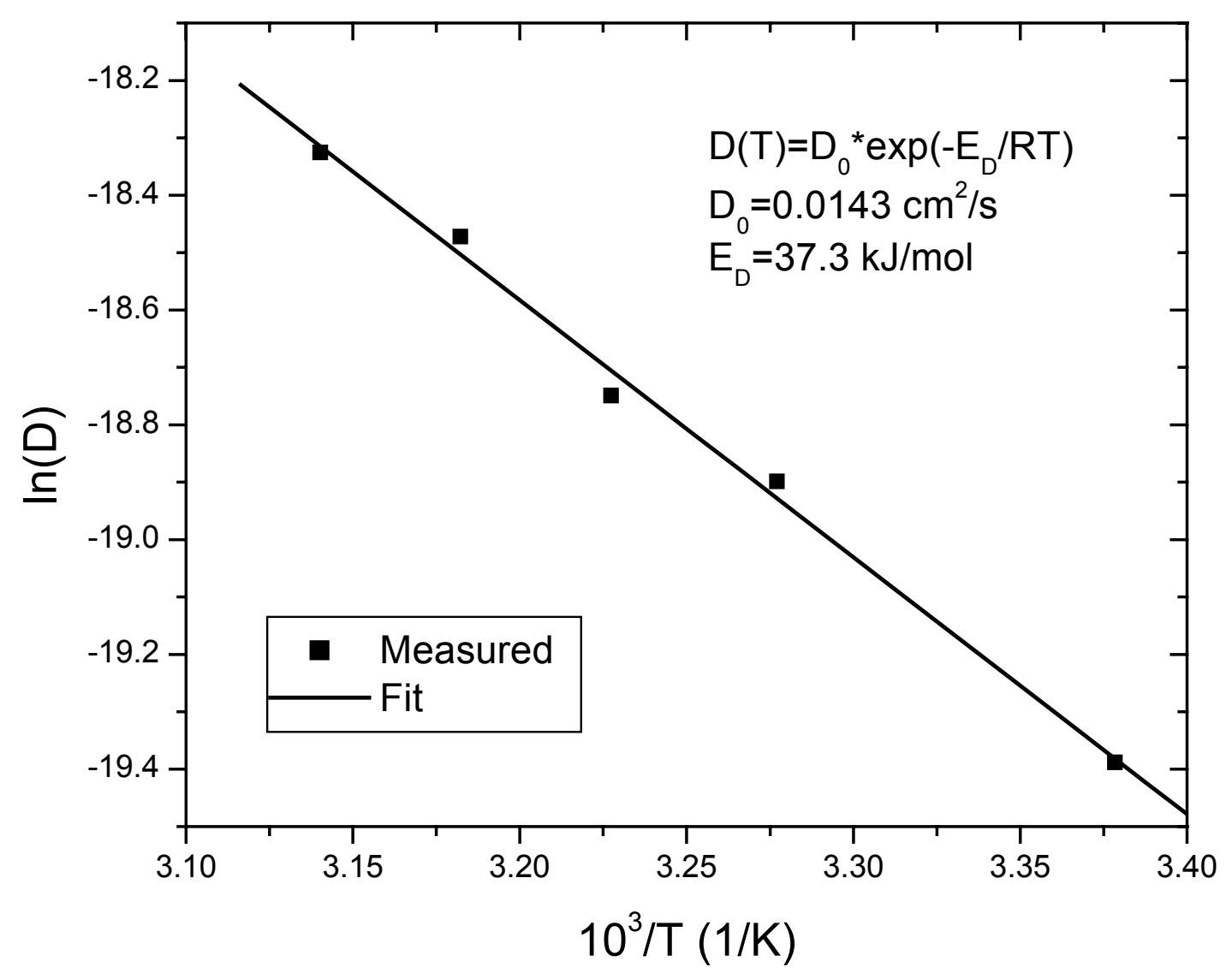

Bao and Dorgan Fig 8 


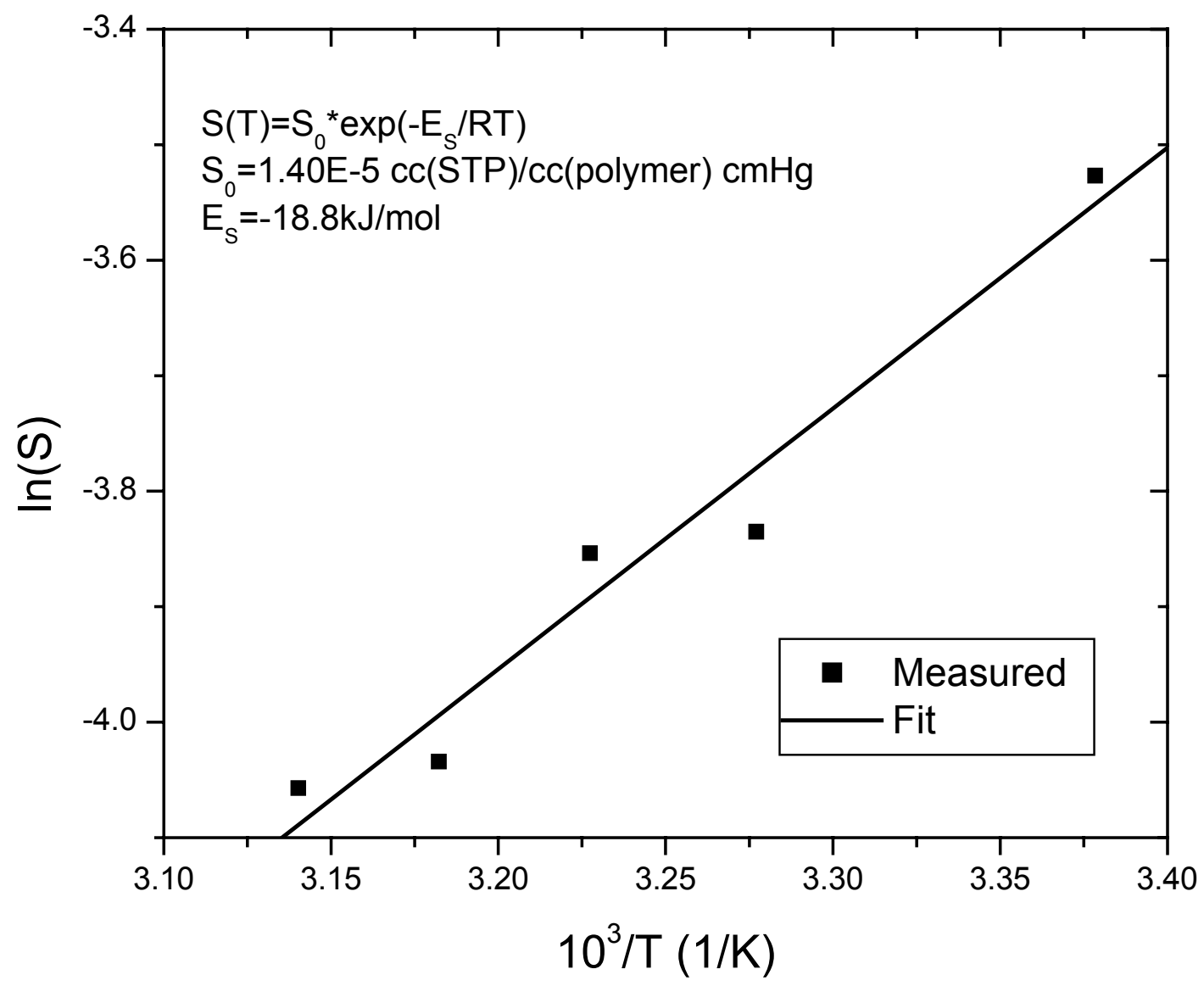

Bao and Dorgan Fig 9 


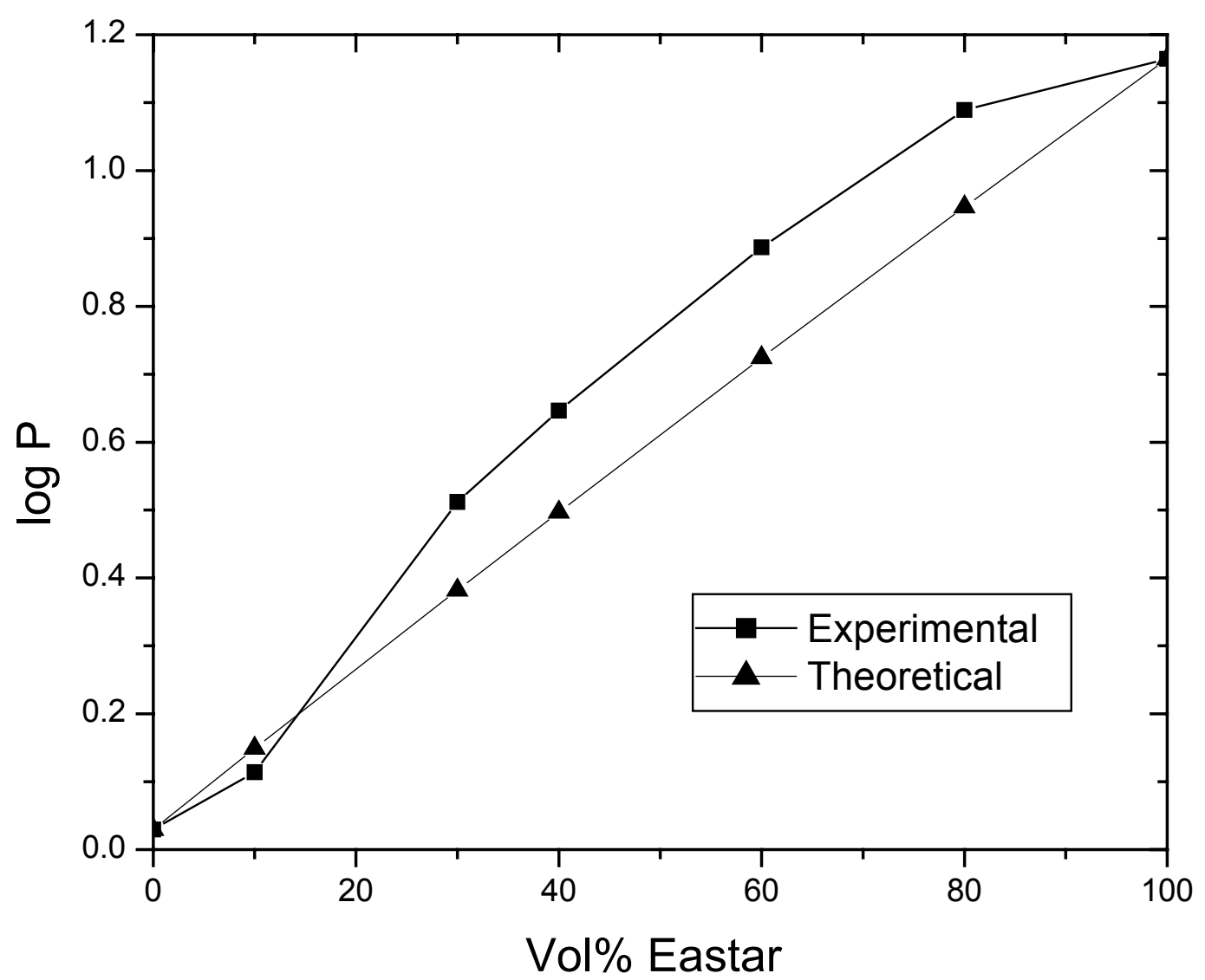

Bao and Dorgan Fig 10 


\title{
Gas Permeation Properties of Poly(lactic acid) Revisited
}

\author{
Lihong Bao ${ }^{\mathrm{a}}$, John R. Dorgan ${ }^{\mathrm{a},{ }^{*}}$, J. Douglas Way ${ }^{\mathrm{a}}$, Dan Knauss ${ }^{\mathrm{b}}$, Sukendu Hait ${ }^{\mathrm{b}}$ \\ Colorado School of Mines \\ Golden, CO 80401
}

\begin{abstract}
Pure gas permeation through solution-cast amorphous poly(lactic acid) (PLA) films is investigated using the time-lag method. New data on permeability, diffusivity and solubility for $\mathrm{N}_{2}, \mathrm{O}_{2}$ and $\mathrm{CO}_{2}$ are reported. At $30{ }^{\circ} \mathrm{C}, \mathrm{N}_{2}$ permeability, diffusivity and solubility in PLA having a stereochemical L:D composition of $98.7: 1.3 \%$ are 0.05 Barrer, $2.4 \mathrm{E}-8 \mathrm{~cm}^{2} / \mathrm{s}$, and $2.2 \mathrm{E}-4$ $\mathrm{cm}^{3}(\mathrm{STP}) / \mathrm{cm}^{3}$ (polymer) $\mathrm{cmHg}$ and the activation energy of permeation is $34.6 \mathrm{~kJ} / \mathrm{mol}$. For $\mathrm{O}_{2}$, the corresponding values are 0.26 Barrer, $5.7 \mathrm{E}-8 \mathrm{~cm}^{2} / \mathrm{s}, 4.9 \mathrm{E}-4 \mathrm{~cm}^{3}(\mathrm{STP}) / \mathrm{cm}^{3}$ (polymer) $\mathrm{cmHg}$ and $24.0 \mathrm{~kJ} / \mathrm{mol}$. The values for $\mathrm{CO}_{2}$ are 1.10 Barrer, $4.4 \mathrm{E}-9 \mathrm{~cm} / \mathrm{s}, 0.025$ $\mathrm{cm}^{3}(\mathrm{STP}) / \mathrm{cm}^{3}$ (polymer) $\mathrm{cmHg}$ and $18.0 \mathrm{~kJ} / \mathrm{mol}$. These new measurements of the gas permeation properties of poly(lactic acid) (PLA) show considerable disagreement with previously reported results (J.M.S., 190 (2001) 243-251) obtained using a continuous flow apparatus. The effect of PLA optical compositions on permeation is also investigated and found to be consistent with the earlier report; the L/D ratio has no effect on the permeation properties provided the material is quenched into an amorphous state. The ability to generate diffusivity and solubility data along with the greater sensitivity of pressure measurement over gas composition argue in favor of the time-lag permeability measurements and suggest that the revised PLA permeation values reported here should be adopted.
\end{abstract}

Key words: Poly(lactic acid); Polylactides; PLA; Permeation; Time-lag; Solubility; Diffusivity

* author to whom correspondence should be addressed. E-mail address: jdorgan@mines.edu

a Chemical Engineering Department

b Chemistry Department 


\section{Introduction}

Poly(lactic acid) (PLA) has received increased attention in the last decade due to its natural biodegradability and availability from renewable resources. (1, 2) PLA can replace hydrocarbon-based polymers such as polystyrene in certain packaging applications and can also be used as a textile material. Full-scale commercial production of PLA is now a reality; the cost of production has been reduced significantly and PLA is being used in diverse applications such as fibers, films, and packaging. $(3,4)$ Therefore, there is a continuing need for a clear understanding of the permeation properties of PLA.

PLA is reportedly a relatively poor barrier to water vapor and $\mathrm{CO}_{2}$. The water-vapor transmission rate of PLA is significantly higher than for PET, PP or PVC. (5) Recently, Auras et. al. investigated barrier properties of two biaxially-oriented PLA films (Cargill-Dow PLA 4030-D and PLA 4040-D). (6) The stereochemical content of PLA 4030-D is 98\% L - for PLA4040-D, it is $94 \% \mathrm{~L} . \mathrm{CO}_{2}$ permeability and activation energy of permeation in PLA $4030-\mathrm{D}$ at $25{ }^{\circ} \mathrm{C}$ are reported as 1.9 Barrers and $15.65 \mathrm{~kJ} / \mathrm{mol}$; in PLA 4040-D, the values are 1.4 Barrers and 19.44 $\mathrm{kJ} / \mathrm{mol} . \mathrm{O}_{2}$ permeability and permeation activation energy in PLA 4030-D at $25{ }^{\circ} \mathrm{C}$ are reported as 0.11 Barrers and $41.43 \mathrm{~kJ} / \mathrm{mol}$, in PLA 4040-D, 0.13 Barrers and $28.43 \mathrm{~kJ} / \mathrm{mol}$. Cargill-Dow, a PLA manufacturer, reports barrier properties of their biaxially-oriented films as follows: the permeability of $\mathrm{O}_{2}$ is $550 \mathrm{cc}-\mathrm{mil} / \mathrm{m}^{2}$ day atm $(\sim 0.21$ Barrers $)$, the $\mathrm{CO}_{2}$ permeability is $3000 \mathrm{cc}-$ $\mathrm{mil} / \mathrm{m}^{2}$ day atm ( 1.2 Barrers). (4) No diffusivity or solubility data for gases in PLA have ever been reported.

In a previous work, the permeation of nitrogen, oxygen, carbon dioxide, and methane in very thin $(5 \mu \mathrm{m})$ amorphous films of various grades of PLA (L:D ratios from 95:5 to 98:2) cast from solution was examined using a flow through geometry and gas chromatography detection. (7) At 
$30{ }^{\circ} \mathrm{C}, \mathrm{N}_{2}$ permeation in PLA was reported as 1.3 Barrers and the permeation activation energy as $11.2 \mathrm{~kJ} / \mathrm{mol}$. For $\mathrm{O}_{2}$ the corresponding values are 3.3 Barrers and $11.1 \mathrm{~kJ} / \mathrm{mol}$ while the reported values for carbon dioxide permeation are 10.2 Barrers and $6.1 \mathrm{~kJ} / \mathrm{mol}$. It was also shown that changes in polymer chain branching and L:D ratios have no effect on the permeation properties of small gases. Compared to the more recent studies cited in the last paragraph, these permeabilities are about an order of magnitude higher. Thus, there exists a need to carefully examine the existing data, present new independent measurements, and come to consensus values for the permeation properties of PLA. In addition, diffusivity or solubility data are desirable. Finally, the L:D ratios of the PLA samples reported on to date were limited to a very small range. This paper thus revisits permeation properties of PLA in order to: (1) rectify the discrepancy in permeability data; (2) find diffusivity and solubility data for gases in PLA, and (3) investigate the effects of $\mathrm{L}: \mathrm{D}$ ratios on the permeation over a wider range.

\section{Materials and Methods}

PLAs used in the experiments were either synthesized or obtained from Cargil-Dow (Minnetonka, Minnesota USA). Table 1 gives the L:D content, molecular weight, and glass transition temperatures $(T g)$. The samples denoted by SH-PLA were synthesized using stannous octanoate-catalyzed ring-opening polymerization. L-lactide and D-lactide (Purac Biochem, Holland) were crystallized from toluene. Tin-2-ethylhexanoate (Aldrich Chemical Co., USA) was distilled under vacuum before use. Benzyl alcohol (Aldrich Chemical Co., USA) was dried with $\mathrm{CaO}$ and distilled under reduced pressure. All polymerizations were performed using the following procedure, with different ratios of L- to D-lactide and amounts of benzyl alcohol.

Lactide $(50 \mathrm{~g}, 0.347 \mathrm{~mol})$ and $\mathrm{Sn}(\mathrm{Oct})_{2}(5.63 \mathrm{mg}, 0.138 \mathrm{mmol})$ were added to a $50 \mathrm{~mL}$ round-bottom flask, dried under vacuum for $4 \mathrm{~h}$, sealed under a rubber septum, and then purged 
with argon for $20 \mathrm{~min}$. Initiator (benzyl alcohol) was added using a syringe in the amount needed to reach the targeted molecular weight. The polymerization reaction was performed by heating the vessel at $130{ }^{0} \mathrm{C}$ for $12 \mathrm{~h}$ under argon atmosphere, with magnetic stirring. The resulting polymer was dissolved in dichloromethane, precipitated with a 10-fold excess of hexane, and dried under reduced pressure (1 torr) for $48 \mathrm{~h}$.

Reagent grade methylene chloride that was $99.6 \%$ pure was obtained from Aldrich and used as received for the membrane casting solvent. High purity gases were obtained from General Air (Denver, CO USA); $\mathrm{N}_{2}$ was $99.999 \%$ pure; $\mathrm{CO}_{2}$ was $99.99 \%$ pure; and $\mathrm{O}_{2}$ was $99.993 \%$ pure.

PLA films were cast from a $10 \mathrm{wt} \%$ solution in methylene chloride using a Gardner casting blade onto a glass plate. Solvent was slowly evaporated for several hours from the film, sometimes with the aide of a cover. Films were subsequently floated off the glass plate onto a water surface, recovered, and dried overnight in a vacuum oven at room temperature.

Various methods for measuring permeability of polymer films have been described in the literature. The time lag method, which allows measurement of permeability, diffusivity and solubility data simultaneously, is described only briefly here; additional details are available. (8) The film is degassed under vacuum for between 12 and 16 hours. Penetrant gas is then introduced on the upstream side at constant pressure $\left(p_{f}\right)$. Depending on the rate of permeation, a variable downstream volume may be affixed. Under these conditions, the amount of permeant per unit area that has permeated through the membrane, $Q_{t}$, is given by

$$
\frac{Q_{t}}{L c_{f}}=\frac{D t}{L^{2}}-\frac{1}{6}-\frac{1}{\pi^{2}} \sum_{n=1}^{\infty} \frac{(-1)^{2}}{n^{2}} \exp \left[-\frac{D n^{2} \pi^{2} t}{L^{2}}\right]
$$

where $L$ is the membrane thickness, $c_{f}$ is the upstream concentration, $D$ is the diffusivity, and $t$ is time. Assuming equilibrium at the upstream interface and that the gas concentration is low enough that Henry's law is valid, gives 


$$
c_{f}=S p_{f}
$$

where $S$ is the Henry's law coefficient or solubility. Using the ideal gas law,

$$
p_{p} V_{p}=\left(Q_{t} A\right) R T
$$

where $p_{p}$ and $V_{p}$ refer to the downstream pressure and volume, respectively and $A$ is the area of the membrane. Accordingly, $Q_{t}$ is obtained from measurement of $p_{p}$ as a function of time. At long time, Equation (1) can be written as,

$$
\frac{L Q_{t}}{p_{f}}=D S\left(t-\frac{L^{2}}{6 D}\right)=P\left(t-\frac{L^{2}}{6 D}\right)
$$

where $P$ is the permeability, defined as the product of diffusivity and solubility. When $L Q_{t} / p_{f}$ is plotted against time, Equation (4) shows that the asymptote of the resulting curve makes an intercept of $L^{2} / 6 D$ on the $t$ axis and has a slope of $P$. This intercept on the $t$ axis is the time lag, $\theta$. Knowing $D$ and slope, $P$, allows calculation of solubility, $S=P / D$. Additional details of the present apparatus can be found elsewhere. (9)

\section{Results and Discussion}

To verify the operation of the time-lag apparatus, $\mathrm{N}_{2}$ permeation through low density polyethylene films provided by Dow Chemical (Midland, MI) was measured. Data are shown in Figure 1. The measured $\mathrm{N}_{2}$ permeability and diffusivity at $25{ }^{\circ} \mathrm{C}$ are 0.90 Barrer and $3.6 \mathrm{E}-7$ $\mathrm{cm}^{2} / \mathrm{s}$. The permeation activation energy is $49.6 \mathrm{~kJ} / \mathrm{mol}$. Corresponding literature values are 0.97 Barrer, 3.2E-7 cm $/ 2$ and $49.4 \mathrm{~kJ} / \mathrm{mol}$ respectively.(10) Accordingly, these measured values are in very good agreement with established literature values.

Gas permeation and sorption properties of glassy and amorphous polymers are particularly sensitive to the film preparation protocol and physical aging. (11) PLA has a relatively low glass transition temperature near $55{ }^{\circ} \mathrm{C}$ (see Table 1) and is known to undergo significant physical 
aging at room temperature. When films physically age, the permeability decreases. This is generally associated with increasing densification and decreasing free volume. Verification of reproducibility during preliminary stages of this work demonstrated that PLA permeability decreases gradually with time, presumably reflecting physical aging. Accordingly, a procedure was developed to investigate the aging effects on the permeation. The PLA film was prepared as described in Section 2.2 and then permeation measurements were carried out as temperature was increased from room temperature to about $45^{\circ} \mathrm{C}$. The PLA film was then annealed at a temperature $5^{\circ} \mathrm{C}$ below its glass transition temperature for $12 \mathrm{hrs}$. After annealing, permeation measurements are taken in a temperature cycle from higher $\left(\sim 45{ }^{\circ} \mathrm{C}\right)$ to lower (ambient) and back to the higher temperature. Figure 2 shows the measured $\mathrm{CO}_{2}$ permeability in PLA 270-60-3 during this procedure. It is easily seen that annealing gives a decrease in permeability of around $20 \%$. Optical microscopy showed no evidence of increased crystallinity resulting from this subTg annealing process. Annealing leads to path independent and reproducible results. Therefore, all the results reported in the paper are from films annealed according to this procedure.

All films were checked for evidence of crystallinity by optical microscopy and differential scanning calorimetry (DSC). PLA 270-60-3 films showed evidence of a small degree of crystallinity under cross-polarized light both before and after annealing. DSC confirmed that less than $4 \%$ crystallinity exists in these films. A high crystallinity PLA 270-60-3 film was prepared for comparison by annealing under vacuum at $110{ }^{\circ} \mathrm{C}$ for 24 hours, crystallinity was very apparent by optical microscopy and DSC showed $40+\%$ crystallinity. It can be concluded that films before and after the permeation tests have low crystallininty; this finding is consistent with the previous measurements.(7) For films of SH-PLA-19 and SH-PLA-43 (L:D = 80:20 and 50:50, respectively), no crystallites were found, consistent with a totally amorphous material. 
Time lag data for carbon dioxide $\left(\mathrm{CO}_{2}\right)$ permeation in PLA 270-60-3 at different temperatures are shown in Figure 3. The membrane thickness is $50.5 \mu \mathrm{m}$ and the time lags are in the range of 450 to 1300 seconds thus allowing $\mathrm{CO}_{2}$ diffusivity to be easily measured with good accuracy. As the temperature increases, the lag time becomes shorter because diffusivity increases with temperature. According to Equation (1), steady-state conditions are effectively reached after a period of three multiples of the time lag. All the experiments leading to the results reported here were carried out for at least five or six multiples of the time lag. Results are examined using linear regression to ensure that the linear region has been reached prior to determining a permeability.

Figures 4-6 detail the temperature dependence of $\mathrm{CO}_{2}$ permeation in PLA 270-60-3. Figure 4 shows the temperature dependence of $\mathrm{CO}_{2}$ permeability. At $30{ }^{\circ} \mathrm{C}$, the permeability is 1.1 Barrers; permeability increases with temperature. Error analysis based on the uncertainties in the measured variables provides the error bars shown in the plot; as shown, repeated measurements at the lowest temperature all fall within the expected range. A very good linear relationship between $\ln P$ and $1 / T$ is found; the activation energy for $\mathrm{CO}_{2}$ permeation is $18.5 \mathrm{~kJ} / \mathrm{mol}$. Figure 5 shows the temperature dependence of $\mathrm{CO}_{2}$ diffusivity. At $30^{\circ} \mathrm{C}, \mathrm{CO}_{2}$ diffusivity is $4.4 \mathrm{E}-9 \mathrm{~cm}^{2} / \mathrm{s}$, which is a typical value for this diffusivity in amorphous/glassy polymers. Diffusivity also increases with temperature and a good linear relationship exists between $\ln D$ and $1 / T$. The corresponding activation energy of diffusion is $36.7 \mathrm{~kJ} / \mathrm{mol}$. Temperature dependence of $\mathrm{CO}_{2}$ solubility, determined from the time lag experiment, is shown in Figure 6. Solubility decreases with temperature as expected. At $30{ }^{\circ} \mathrm{C}, \mathrm{CO}_{2}$ solubility is $0.025 \mathrm{~cm}^{3}(\mathrm{STP}) / \mathrm{cm}^{3}$ (polymer) $\mathrm{cmHg}$. The activation energy is negative, and the value is $-18.2 \mathrm{~kJ} / \mathrm{mol}$. The independent $\mathrm{CO}_{2}$ diffusivity and solubility in PLA have never been reported before, so a direct comparison with 
literature values is not possible. For PLA with different L:D ratios, $\mathrm{CO}_{2}$ permeation behaves similarly - results are tabulated in Tables 2 and 3.

Figure 7 shows time lag plot for $\mathrm{O}_{2}$ permeation in PLA (L:D $\left.=80: 20\right)$ at $22.8{ }^{\circ} \mathrm{C}$. In this experiment, the membrane thickness is $50.0 \mu \mathrm{m}$. The time lag is about 150 seconds, an order of magnitude lower than that of $\mathrm{CO}_{2}$. The permeability is 0.14 Barrer, diffusivity is $3.6 \mathrm{E}-8 \mathrm{~cm}^{2} / \mathrm{s}$, and solubility is $3.8 \mathrm{E}-4 \mathrm{~cm}^{3}(\mathrm{STP}) / \mathrm{cm}^{3}$ (polymer) $\mathrm{cmHg}$. The permeation activation energy is $24.9 \mathrm{~kJ} / \mathrm{mol}$. Results are not strongly dependent on the L:D ratios as evidenced in the summary of Tables 2 and 3.

It is well known that $\mathrm{N}_{2}$ has lower diffusivity and permeability compared to $\mathrm{O}_{2}$ in most polymer films, which can serve as the basis of air separation. PLA behaves as expected; the measured $\mathrm{N}_{2}$ permeability in PLA ( $\mathrm{L}: \mathrm{D}=98.7: 1.3$ ) is about 0.05 Barrer, 5 times smaller than $\mathrm{O}_{2}$. This small permeation is difficult to measure accurately. The time lags are only a few seconds for all the films of thickness around $50 \mu \mathrm{m}$, so accurate measurement of diffusivity is very difficult. One way to solve this problem is to increase the film thickness. Measurements were done using PLA ( $\mathrm{L}: \mathrm{D}=80: 20)$ film with a thickness of $154.0 \mu \mathrm{m}$. It was found that even with this increased film thickness, the time lag is short and it is hard to get meaningful diffusivity data. One complication associated with increased film thickness is that even the permeation becomes hard to measure. For nitrogen, it was not possible to measure separate diffusivity and solubility contributions to the permeability.

Table 2 summarizes the results for $\mathrm{N}_{2}, \mathrm{O}_{2}$ and $\mathrm{CO}_{2}$ permeation properties (Permeability, Diffusivity and Solubility) in PLAs with three different L/D ratios. This Table contains results from the time lag and the ideal solubility and diffusivity for comparison. The ideal solubility of a gas in a polymer is calculated according to, (12) 


$$
S=\frac{1}{2.72 p_{i, S a t}}
$$

where $p_{i, S a t}$ is the saturation pressure of the permeating gas. The saturation pressures are calculated using the Antoinne equation or similar correlations. The ideal diffusivity is obtained by dividing the measured permeability by the ideal solubility. The activation energies for the measured solubilities are listed in Table 3. The summary of property values reported in Table 2 deserves further comment.

Error analysis of the time lag apparatus reveals that for $\mathrm{CO}_{2}$ permeation, the maximum uncertainties of the given results for permeability, diffusivity and solubility are about $4 \%, 6 \%$ and $7 \%$, respectively. For $\mathrm{O}_{2}$ permeation, the values increase to $7 \%, 12 \%$ and $14 \%$. For $\mathrm{N}_{2}$ permeation, the values are $7 \%, 16 \%$ and $18 \%$. The major source of uncertainty is from the nonuniformity of the membrane thickness. Another measurement issue is the inherent system leaks. For $\mathrm{CO}_{2}$ permeation, the system leak rate (around $10^{-7} \mathrm{~cm}^{3} / \mathrm{s}$ ) is much smaller than the permeation rate and the long time lag means both permeability and diffusivity can be measured accurately. However, for $\mathrm{N}_{2}$, the permeation rate is small, so the leaking becomes more significant and results in less accurate permeability measurements. Also, the very short time lag makes it harder to get accurate measurements of diffusivity. That is the reason why some diffusivity data are not reported in Table 2 .

The new permeability data are about one order of magnitude lower than the previously reported results, (7) but much closer to the reported permeability of biaxially-oriented films. (6) Even the present pre-annealed films show much lower permeation than previously reported. One possible explanation is that previous results were obtained using a flow through system with gas chromatography detection necessitating the use of very thin PLA films - only about $5 \mu \mathrm{m}$ in thickness. Such a thin film is very hard to handle; structural defects such as microscopic 
pinholes or cracks could be formed in the film. Additionally, these thin films were not annealed and may have considerably different characteristics with respect to physical aging. Also, the presence of residual solvent may have affected the permeation. Finally, the small permeation rates may have caused problems in the legitimacy of the GC calibration curves.

Since diffusivity and solubility data for PLA are not reported in the literature, the results reported here represent new findings and provide new insight. Measured time lag permeabilities are divided by the ideal solubility to determine a $D_{\text {ideal }} . D_{\text {ideal }}$ is the same order of magnitude as the time lag diffusivity, $D_{\theta}$, and both are typical for diffusion of gases in polymer glasses. However, $D_{\text {ideal }}$ does not follow the usual decreasing order with increasing molecular size of the gases ( $D_{\mathrm{CO}_{2}}<D_{\mathrm{N}_{2}}<D_{\mathrm{O}_{2}}$ ). The time lag diffusivities do follow the appropriate order. That is, the experimentally determined diffusivities are internally consistent. It is concluded that the time lag measurements yield reasonable solubilities that can be used to better understand the permeation properties of gases in PLA. Relative to the "ideal" values which do not account for gas-polymer energetic interactions, the solubility of $\mathrm{CO}_{2}$ is large whereas the solubilities of $\mathrm{N}_{2}$ and $\mathrm{O}_{2}$ are of the expected order or slightly lower. These findings should have implications for strategies to control the permeation properties of PLA.

Finally, as all of the samples were solution cast and are amorphous, the effect of optical composition on amorphous phase permeability is small and effectively negligible. It should be remembered though that optical composition does strongly affect achievable crystallintiy in melt processing operations or when samples are annealed. Under such conditions, permeability is usually dominated by the level of crystallinity in the samples although the density of amorphous and crystalline PLA are not very different. 


\section{Conclusions}

Due to the discrepancy of previous permeability results and newer literature values, gas permeation properties of PLA were revisited using the time lag method. It was found that annealing the PLA film is necessary for path independent and reproducible results. New permeability values are lower than those previous reported. Separate contributions of the pure gas diffusivities and solubilities in PLA are reported for the first time. Careful examination of the data relative to expected trends with molecular size favor adoption of the time-lag solubility values. Furthermore, the results suggest that the barrier properties of PLA to $\mathrm{O}_{2}$ and $\mathrm{N}_{2}$ are a result of low solubility values while the permeability of $\mathrm{CO}_{2}$ can be associated with a significantly higher solubility. The PLA optical composition has a very small effect on permeation properties confirming previous results.

In order to improve the barrier properties of PLA for some packaging applications, technologies including nanocomposites, aluminizing, or incorporation of other barrier layers are necessary. PLA, being degradable, may also find application in situations where time varying gas permeability is desirable.

\section{Acknowledgements}

Funding was provided by the EPA/NSF Technology for a Sustainable Environment Program (No. R 826733-01-0) and by the Agriculture Industries of the Future Program of the Office of Industrial Technologies, U.S. Department of Energy. 


\section{Bibliography:}

1. Hartman MH. 1998. High Molecular Weight Polylactic Acid Polymers. In Biopolymers from Renewable Resources, ed. D.H. Kaplan, pp. 367-411. Berlin: Springer-Verlag

2. Dorgan JR. 2001. Polylactides: Properties and Prospects of an Environmentally Benign Plastic from Renewable Resources. Macromolecular Symposia 175: 55-66

3. Lunt J. 2000. Poly(lactic acid) Polymers for FIbers and Nonwovens. International Fiber Journal: $48-52$

4. Cargill-Dow. 2003 technical data sheets available at www.cdpoly.com.

5. Siparsky GL, Voorhees KJ, Dorgan JR, Schilling K. 1997. Water Transport in Poly(lactic acid), Poly(lactic acid-co-caprolactone) Copolymers, and Poly(lactic acid) / Polyethylene Glycol Blends. Journal of Environmental Polymer Degradation 5: 125-36

6. Auras RA, Harte B, Selke S, Hernandez RJ. 2002. Mechanical, physical, and barrier properties of poly(lactic acid) films. Presented at Worldpak-IAPRI, East Lansing, MI. 48824-1224. USA

7. Lehermeier H.J., Dorgan J.R., Way J.D. 2001. Gas Permeation Properties of Poly(lactic acid). J. Memb. Sci. 190: 243-51

8. Hands D. 1999. Chapter 30 - Permeability. In Handbook of Polymer Testing, Physical Methods, ed. R Brown, pp. 747-60. New York

9. Bao L., Tayeb, M., Dorgan J.R. 2003. Design of a time-lag apparatus for permeation measurements in polymer thin films. In Preparation, Rev. Sci. Instr.

10. Brandrup J, Immergut EH, Grullce EA. 1999. Polymer Handbook. New York: John Wiley \& Sons

11. Nagai K, Toy LG, Freeman BD, et al. 2000. Gas permeability and hydrocarbon solubility of poly[1-phenyl-2-[p[-triisopropylsilyl)phenyl]acetylene],. J. Polym. Sci. B: Polymer Physics. 38: $1474-84$

12. Baker RW. 2000. Membrane Technology and Applications. New York: McGraw Hill 
Table 1. Polylactide properties

\begin{tabular}{|c|c|c|c|c|c|}
\hline $\begin{array}{c}\text { Sample } \\
\text { Number }\end{array}$ & $\begin{array}{c}\mathbf{L} \\
\mathbf{( \% )}\end{array}$ & $\begin{array}{c}\mathbf{D} \\
\mathbf{( \% )}\end{array}$ & $\begin{array}{c}\mathbf{M}_{\mathbf{n}} \\
(\mathbf{g} / \mathbf{m o l})\end{array}$ & $\begin{array}{c}\mathbf{M}_{\mathbf{w}} \\
(\mathbf{g} / \mathbf{m o l})\end{array}$ & $\begin{array}{c}\mathbf{T}_{\mathbf{g}} \\
(\mathbf{0} \mathbf{C})\end{array}$ \\
\hline $270-60-3$ & 98.7 & 1.3 & $* * *$ & $1.90 \mathrm{E}+5$ & 59 \\
\hline SH-PLA-19 & 80 & 20 & $9.2 \mathrm{E}+4$ & $1.42 \mathrm{E}+5$ & 54 \\
\hline SH-PLA-43 & 50 & 50 & $8.4 \mathrm{E}+4$ & $1.72 \mathrm{E}+5$ & 52 \\
\hline
\end{tabular}


Table 2. Summary of PLA permeation properties

\begin{tabular}{|c|c|c|c|c|c|c|}
\hline Permeant & $\begin{array}{c}\mathbf{L}: \mathbf{D} \\
\text { Ratio }\end{array}$ & $\begin{array}{c}\mathbf{P}_{\theta} \\
\left.\mathbf{( 3 0}^{\mathbf{0}} \mathbf{C}\right)\end{array}$ & $\begin{array}{c}\mathbf{D}_{\theta} \\
\left.\mathbf{( 3 0}^{\circ} \mathbf{C}\right)\end{array}$ & $\begin{array}{c}\mathbf{D}_{\text {ideal }} \\
\left.\mathbf{( 3 0}^{\circ} \mathbf{C}\right)\end{array}$ & $\begin{array}{c}\mathbf{S}_{\theta} \\
\left.\mathbf{( 3 0}^{\mathbf{0}} \mathbf{C}\right)\end{array}$ & $\begin{array}{c}\mathbf{S}_{\text {ideal }} \\
\left.\mathbf{( 3 0}^{\circ} \mathbf{C}\right)\end{array}$ \\
\hline $\mathbf{C O}_{2}$ & $98.7: 1.3$ & 1.10 & $4.4 \mathrm{E}-9$ & & $2.5 \mathrm{E}-2$ & \\
\hline & $80: 20$ & 0.51 & $3.8 \mathrm{E}-9$ & $9.8 \mathrm{E}-9$ & $1.3 \mathrm{E}-2$ & $5.2 \mathrm{E}-3$ \\
\hline $\mathbf{O}_{2}$ & $90: 50$ & 0.71 & $4.5 \mathrm{E}-9$ & & $1.6 \mathrm{E}-2$ & \\
\hline & $80: 7: 1.3$ & 0.26 & $5.7 \mathrm{E}-8$ & & $4.9 \mathrm{E}-4$ & \\
\hline & $50: 50$ & 0.17 & $7.6 \mathrm{E}-8$ & & $2.2 \mathrm{E}-4$ & \\
\hline $\mathbf{N}_{\mathbf{2}}$ & $98.7: 1.3$ & 0.05 & $2.4 \mathrm{E}-8$ & & $2.2 \mathrm{E}-4$ & \\
\hline & $80: 20$ & 0.02 & $* * *$ & $3.1 \mathrm{E}-9$ & $* * *$ & $6.5 \mathrm{E}-4$ \\
\hline & $50: 50$ & 0.03 & $* * *$ & & $* * 6 * 4$ & \\
\hline
\end{tabular}

(Units: P --- Barrer; D --- $\mathrm{cm}^{2} / \mathrm{s} ; \mathrm{S}$--- $\mathrm{cm}^{3}(\mathrm{STP}) / \mathrm{cm}^{3}$ (polymer) $\mathrm{cmHg}$ ) 
Table 3. Activation energies for permeation of gases in PLA

\begin{tabular}{|c|c|c|c|c|}
\hline Permeant & L/D Ratio & $\mathbf{E}_{\mathbf{P}}$ & $\mathbf{E}_{\mathbf{D}}$ & $\mathbf{E}_{\mathbf{S}}$ \\
\hline $\mathbf{C O}_{\mathbf{2}}$ & $98.7: 1.3$ & 18.1 & 36.3 & -18.4 \\
\hline & $80: 20$ & 17.8 & 32.2 & -13.9 \\
\hline & $50: 50$ & 14.3 & 34.8 & -25.4 \\
\hline $\mathbf{O}_{2}$ & $98.7: 1.3$ & 24.0 & 42.7 & -19.2 \\
\hline & $80: 20$ & 24.9 & 40.8 & -15.9 \\
\hline & $50: 50$ & 26.6 & 68.8 & -42.0 \\
\hline $\mathbf{N}_{2}$ & $98.7: 1.3$ & 34.6 & 59.3 & -25.0 \\
\hline & $80: 20$ & 40.9 & $* * *$ & $* * *$ \\
\hline & $50: 50$ & 35.0 & $* * *$ & $* * *$ \\
\hline
\end{tabular}




\section{Figure Captions}

Figure 1. Typical time lag for $\mathrm{N}_{2}$ permeation in LDPE. The measurement was conducted as a verification of the apparatus.

Figure 2. The measured $\mathrm{CO}_{2}$ permeability in PLA film (L:D = 98.3:1.7) during annealing procedure.

Figure 3. Typical time lags for $\mathrm{CO}_{2}$ permeation in PLA (L:D = 98.7:1.3) under different temperatures.

Figure 4. Temperature dependence of $\mathrm{CO}_{2}$ Permeability in PLA (L:D=98.7:1.3).

Figure 5. Temperature dependence of $\mathrm{CO}_{2}$ Diffusivity in PLA (L:D=98.7:1.3).

Figure 6. Temperature dependence of $\mathrm{CO}_{2}$ Solubility in PLA (L:D = 98.7:1.3).

Figure 7. Time lag plot for $\mathrm{O}_{2}$ permeation in PLA $(\mathrm{L}: \mathrm{D}=80: 20)$. Temperature is $22.8^{\circ} \mathrm{C}$. 


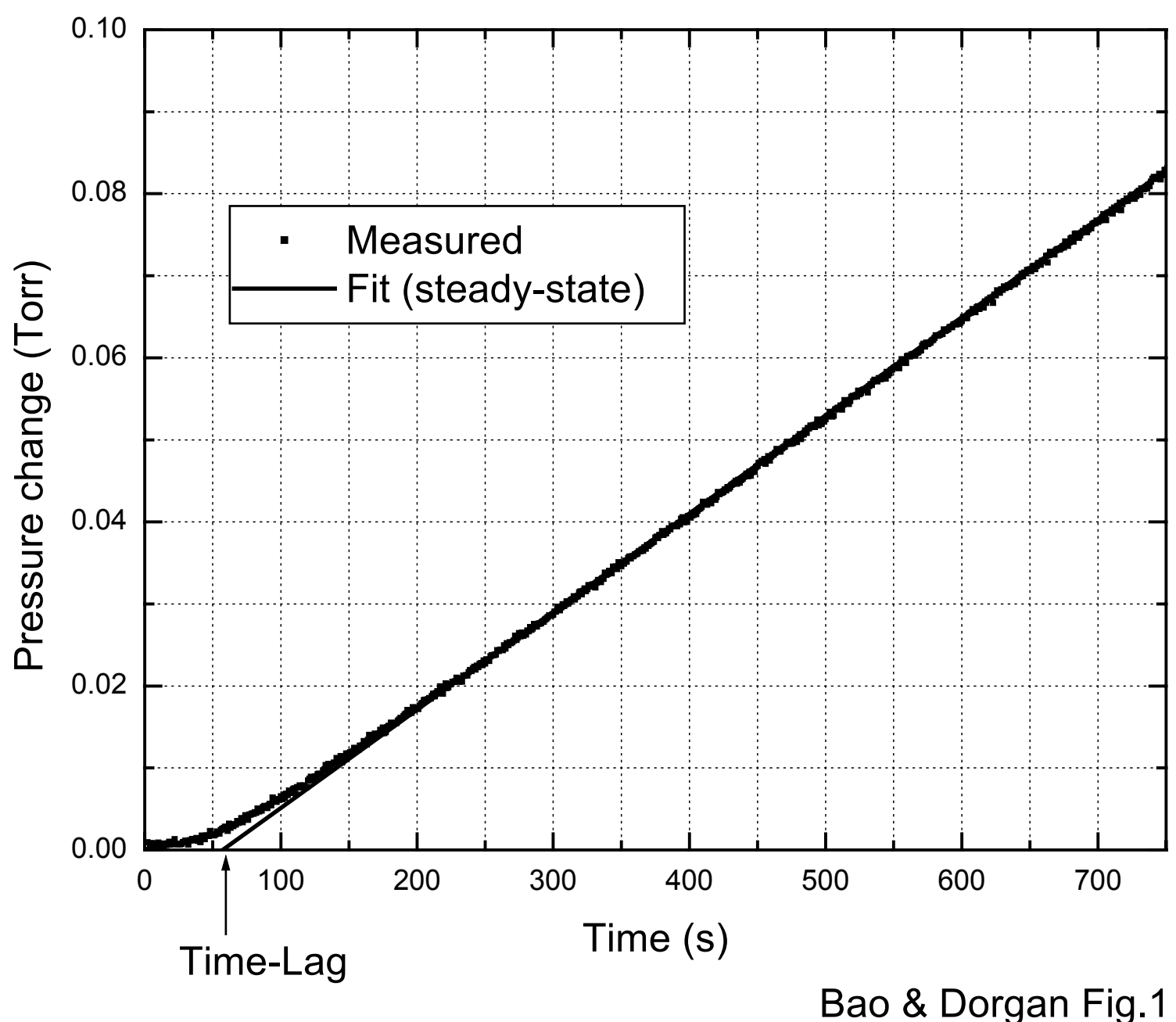




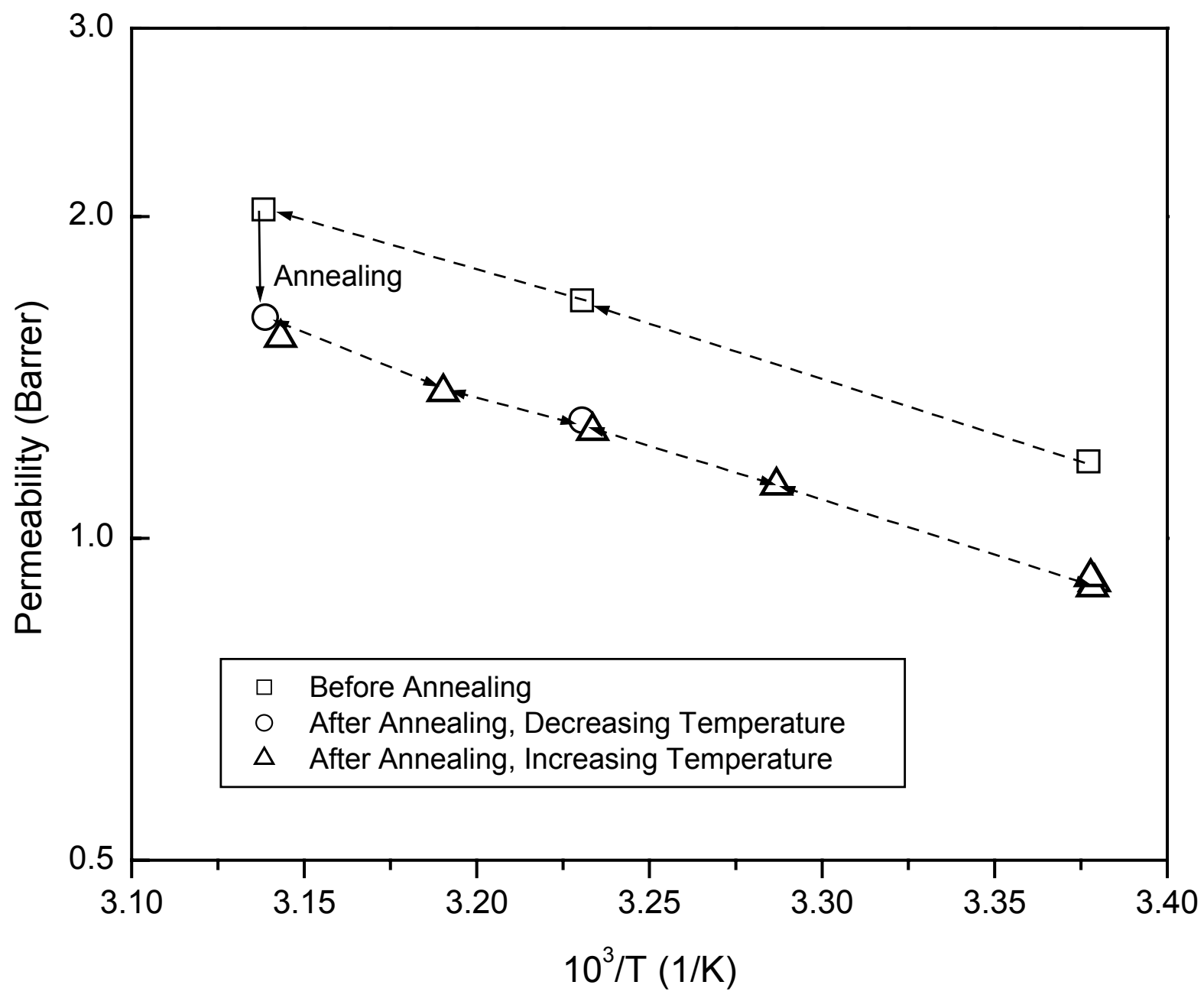

Bao \& Dorgan Fig.2 


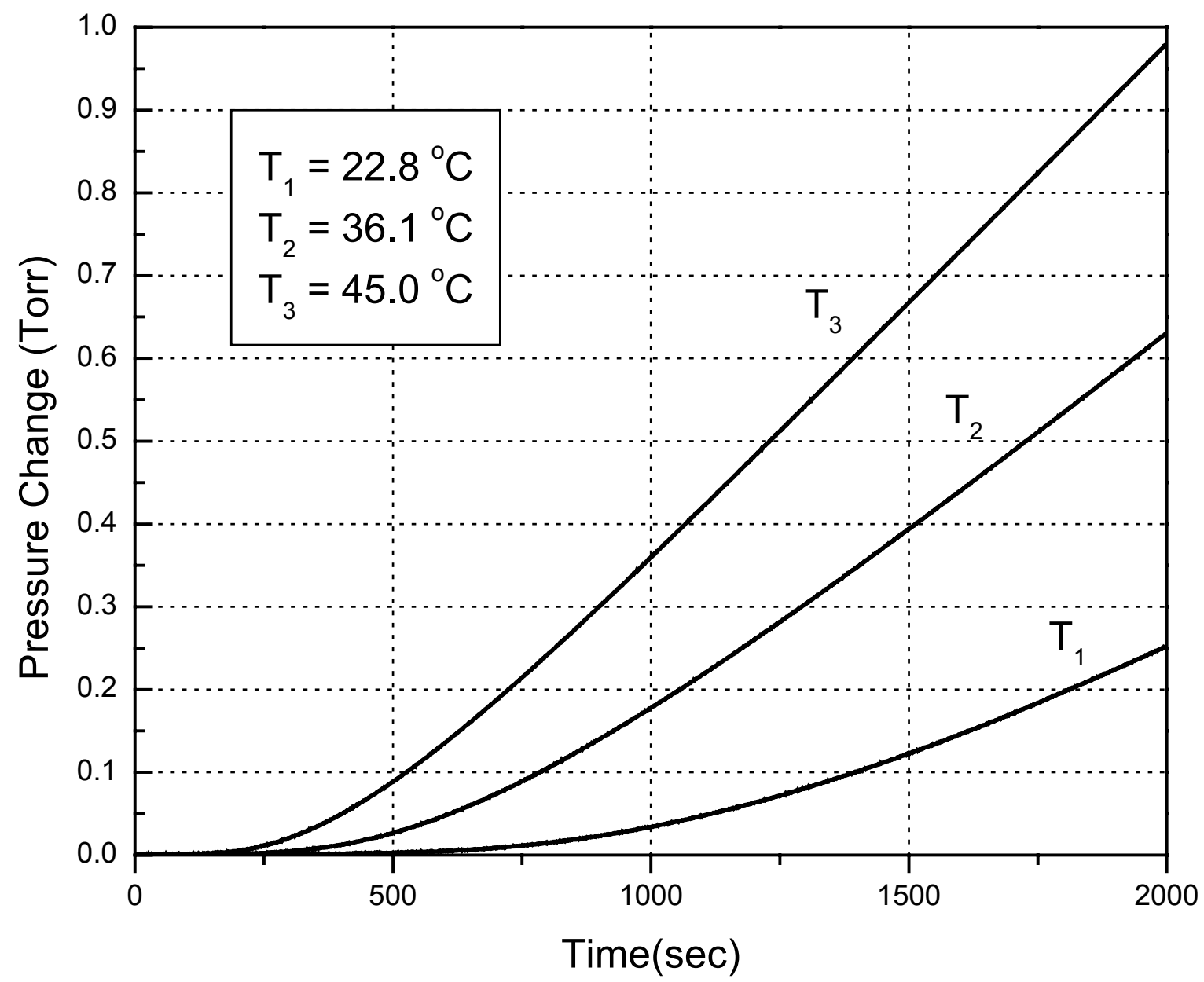

Bao \& Dorgan Fig.3 


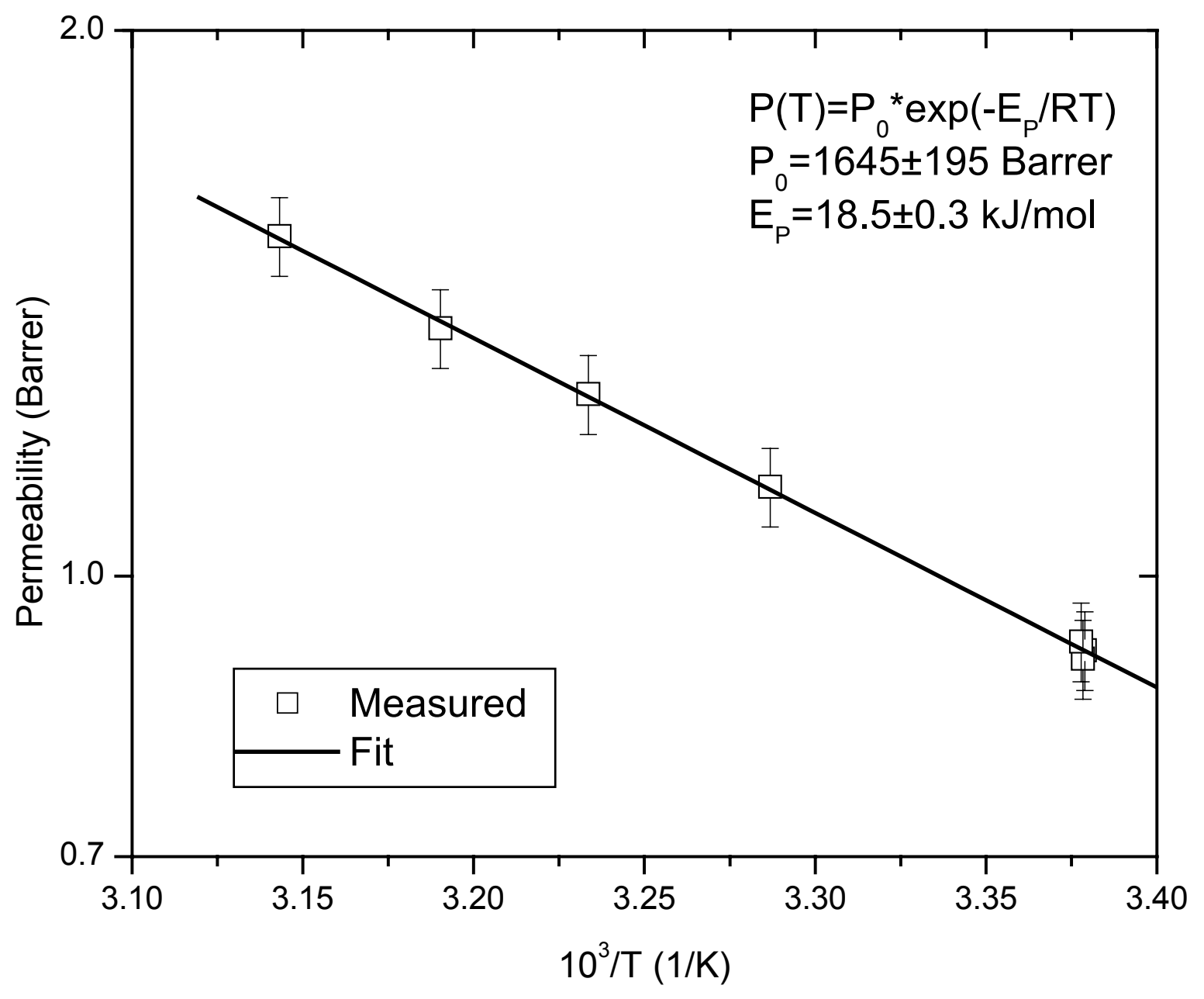

Bao \& Dorgan Fig.4 


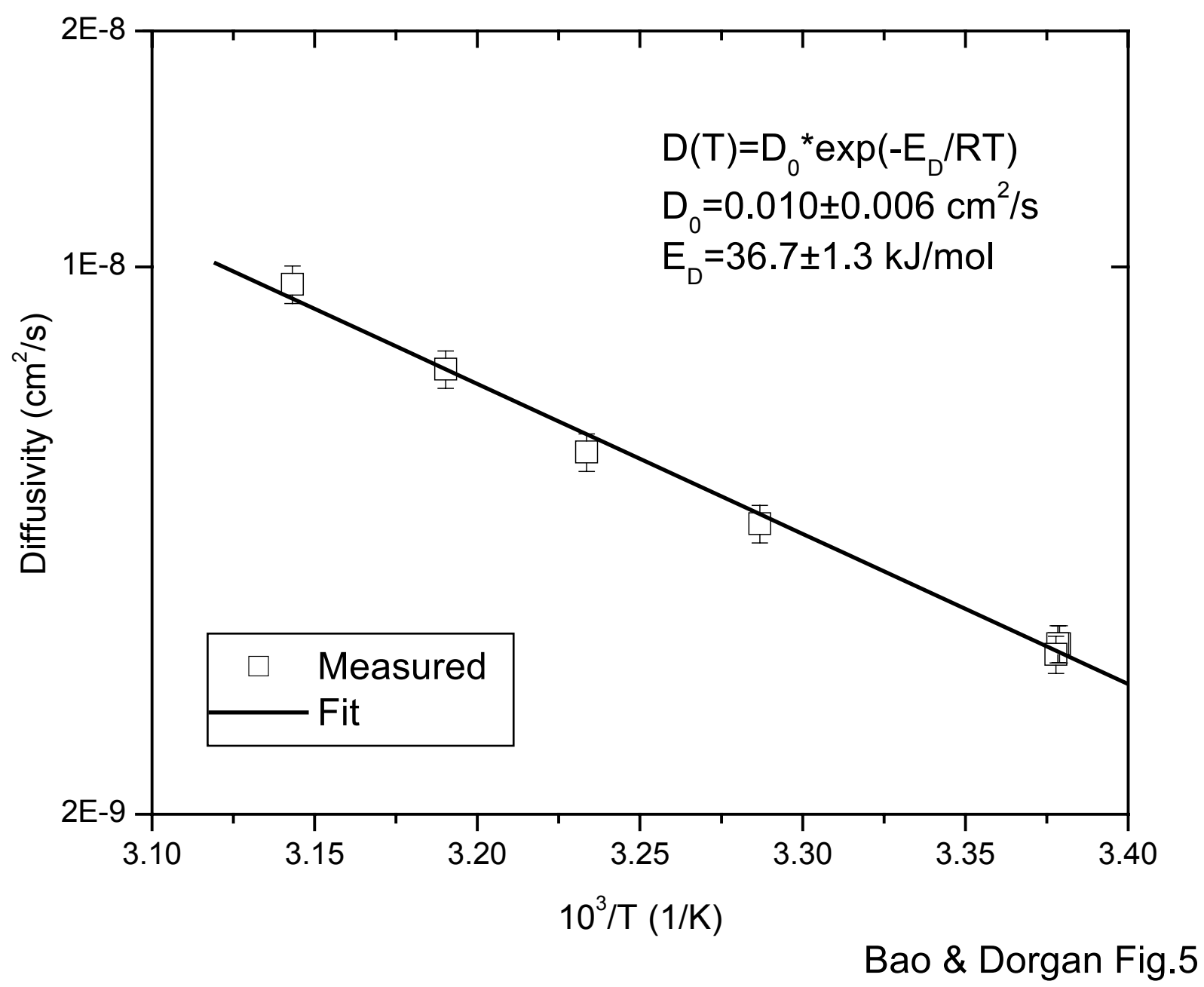




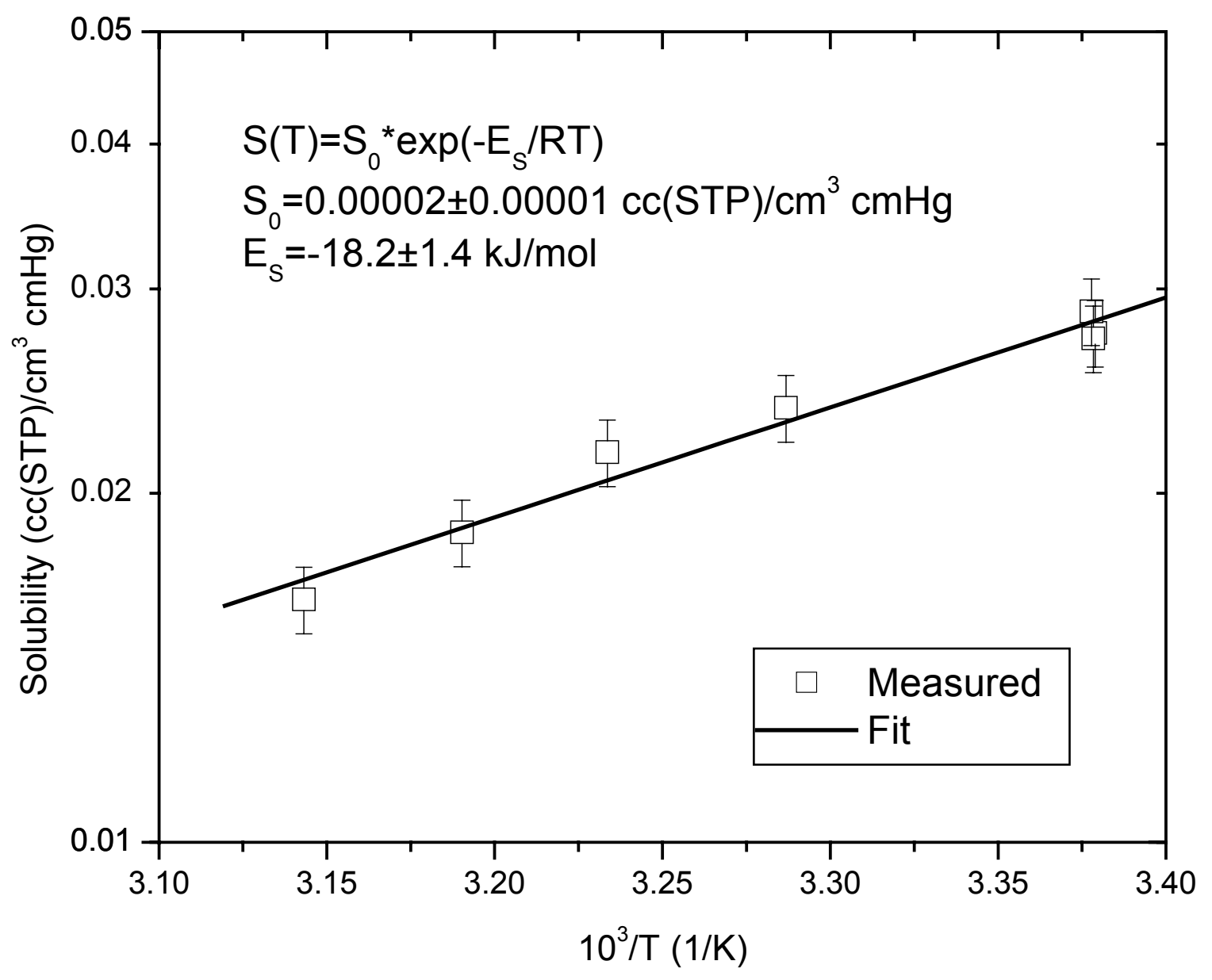

Bao \& Dorgan Fig.6 


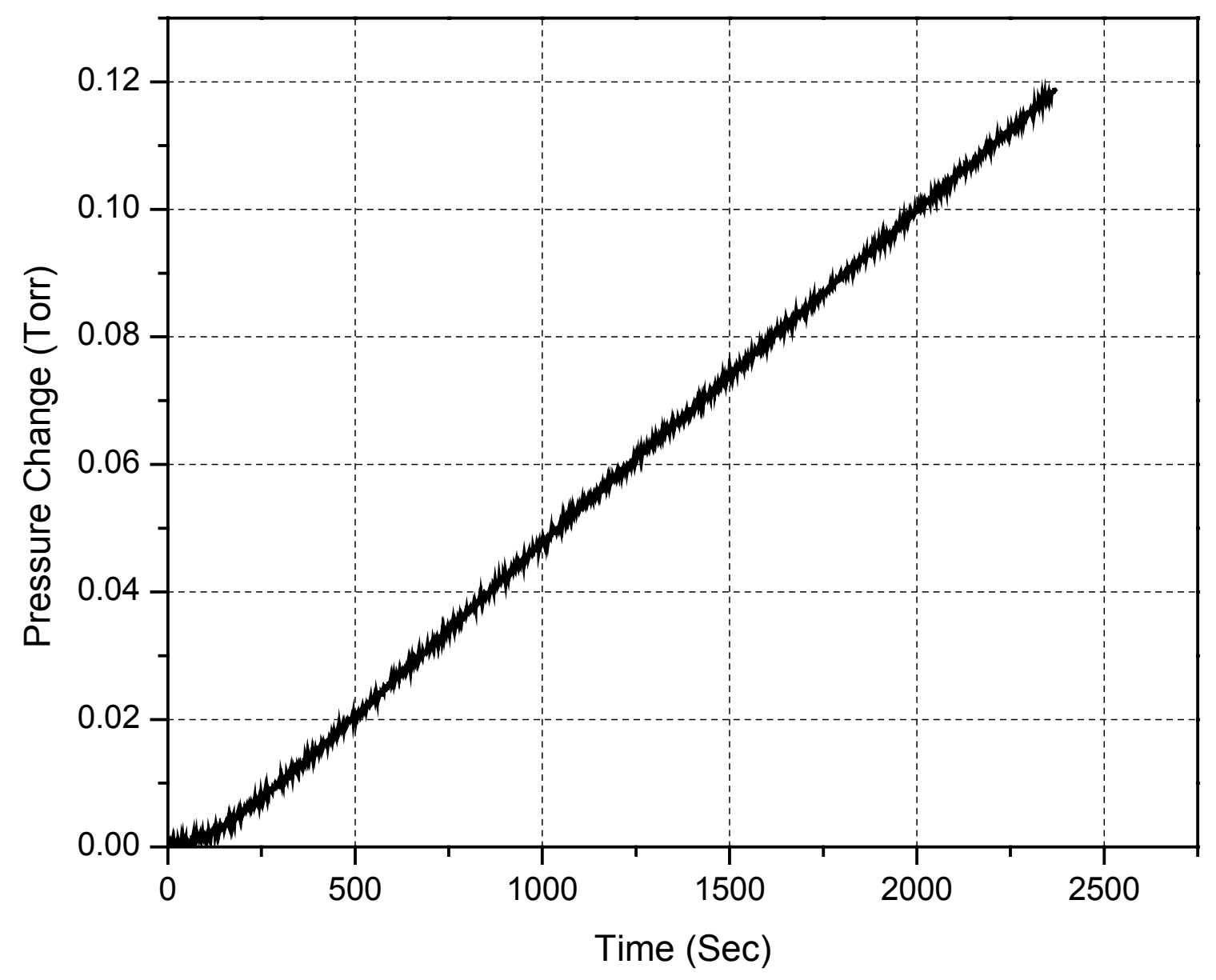

Bao \& Dorgan Fig.7 


\title{
Fundamental Solution and Single-Chain Properties of Polylactides
}

\author{
John R. Dorgan, ${ }^{* \dagger}$ Jay Janzen, ${ }^{\dagger}$ Daniel M. Knauss, ${ }^{*}$, Sukhendu B. Hait, ${ }^{\ddagger}$ Bradford R. Limoges, ${ }^{\dagger}$ \\ and Matthew H. Hutchinson ${ }^{\dagger, \diamond}$ \\ Chemical Engineering Department and Department of Chemistry and Geochemistry \\ Colorado School of Mines, Golden, Colorado 80401
}

\begin{abstract}
Polylactides (PLA) have been known for several decades and have recently become of considerable commercial significance. However, the literature on basic chain properties and solution characterization is divided and inconsistent. In this study, a comprehensive and wellcontrolled set of experiments is combined with rigorous quantitative analysis in order to resolve existing apparent contradictions. Homopolymers and copolymers spanning wide ranges of molecular weight and stereoisomer proportions were prepared by ring-opening polymerizations of L- and D-lactides using stannous octanoate as catalyst. Samples were characterized by means of: 1) dilute-solution viscometry in three different solvents; 2) size-exclusion chromatography in THF with light-scattering detection; 3) static multiangle light scattering in a mixed acetonitriledichloromethane solvent; 4) variable-angle spectroscopic ellipsometry; and 5) melt rheology. The data imply polylactides are typical linear flexible polymers; unperturbed PLA chain dimensions as describable in terms of a characteristic ratio of $6.5 \pm 0.9$, regardless of stereoisomer content. The Schulz-Blaschke and Mark-Houwink constants for dilute PLA solutions in chloroform and in THF are determined. For chloroform at $30{ }^{\circ} \mathrm{C}$ the correct values are $k_{S B}=0.302, K=0.0131(\mathrm{~mL} / \mathrm{g})$ and $a=0.759$ while for THF at $30^{\circ} \mathrm{C}$ the correct values are $k_{S B}=0.289, K=0.0174(\mathrm{~mL} / \mathrm{g})$ and $a=0.736$. Experimental evidence to prefer unperturbed chaindimension (theta-state) estimates based on Stockmayer-Fixman analysis over Orofino-Flory analysis of second virial coefficients is presented.
\end{abstract}

Keywords: polylactides, PLA, poly(lactic acid) intrinsic viscosity, Mark-Houwink, SchulzBlaschke, characteristic ratio, packing model, rheology

\footnotetext{
* Authors for correspondence. e-mail: jdorgan@mines.edu or dknauss@mines.edu

$\dagger$ Chemical Engineering Department

$\$$ Dept. of Chemistry and Geochemistry.

${ }^{\diamond}$ Present affiliation: Dept. of Chemical Engineering, Cambridge University
} 


\section{Introduction}

Polylactides are environmentally benign and degradable polymers[Hartmann, 1998 \#681] made from renewable resources. They have physical properties that make them useful for fibers, packaging, and other applications traditionally dominated by petroleum-based resins. The recent advent of large-scale commercial production ${ }^{\mathrm{a}}$ of these materials makes characterization of their fundamental properties urgently desirable.

Unfortunately, literature reports on some fundamental properties of PLAs seriously disagree. For example, Table XVIII of a recent review[Garlotta, 2001 \#706] lists fifteen different sets of Mark-Houwink (MH) constants,[Kratochvil, 1989 \#48] and agreement among the various sets is poor, even for a single solvent within a modest range of temperature. Evaluation of the $\mathrm{MH}$ values tabulated in the above review can be performed by reconstructing data for the so-called Stockmayer-Fixman extrapolation. This graphical technique consists of plotting the intrinsic viscosity divided by the square root of the viscosity averaged molecular weight against the square root of the viscosity averaged molecular weight and can be used to estimate the theta-condition front factors $\left(K_{\Theta}\right)$ for the $\mathrm{MH}$ equation. Figure 1 shows the $K_{\Theta}$ values (vertical-axis intercepts) to vary over a wide range, from lowest to highest, by a factor of 25 ! Because $K_{\Theta}$ is a fundamental inherent chain property, it can be concluded that the state of the present literature regarding Mark-Houwink parameters is inadequate.

Literature reports[Tonelli, 1969 \#571; Schindler, 1979 \#591; Kim, 1992 \#690; Joziasse, 1996 \#575; Kang, 2002 \#683; Ren, 2003 \#723] that describe experimental determinations of characteristic ratios[Kratochvil, $1989 \# 48]\left(C_{\infty}\right)$ for PLAs reveals several factors contributing to 
the inconsistencies pointed out in the preceding paragraph. These factors include: (1) Different definitions of the mean squared "monomer" step length; (2) assumptions of different expansionfactor models for correcting data obtained in good solvents to $\Theta$ conditions or neglecting to make such correction altogether[Ren, 2003 \#723]; (3) different assumed values for the viscosity function $\Phi$ (or "Flory constant") used to relate intrinsic viscosities to molecular dimensions in solution; and (4) different methods of obtaining molecular weights and subsequently relating them to dilute-solution viscosities.

The present study was undertaken to resolve questions thus raised by previous conflicting reports about the unperturbed chain dimensions of PLAs, and to arrive at some carefully derived MH constants for PLAs in nontheta solvents; reliable MH constants enable accurate characterization of these increasingly important polymers. The work is intended to refine and supplement earlier,[Dorgan, 1999 \#536; Dorgan, 2000 \#562] recent,[Palade, 2001 \#535; Mierzwa, $2002 \# 588]$ and concurrent studies from our laboratories.

\section{Materials and Methods}

For continuity with much existing PLA literature, the traditional labeling of the lactide stereoisomers as "L-" and "D-" instead of the generally preferred $(S, S)$ and $(R, R)$ designations is retained. Homopolymers and copolymers of enantiomeric lactides (cyclic dimers of lactic acid) were synthesized using stannous octanoate-catalyzed ring-opening polymerizations. L-lactide and D-lactide (Purac Biochem., Holland) were crystallized from toluene. Tin-2-ethylhexanoate (Aldrich Chemical Co., USA) was distilled under vacuum before use. Benzyl alcohol (Aldrich Chemical Co., USA) was dried with $\mathrm{CaO}$ and distilled under reduced pressure. All

\footnotetext{
${ }^{\text {a }}$ For details see http://www.cargilldow.com
} 
polymerizations were performed using the following procedure, with different ratios of L- to Dlactide and amounts of benzyl alcohol.

Lactide $(50 \mathrm{~g}, 0.347 \mathrm{~mol})$ and $\mathrm{Sn}(\mathrm{Oct})_{2}(5.63 \mathrm{mg}, 0.138 \mathrm{mmol})$ were added to a $50 \mathrm{~mL}$ round-bottom flask, dried under vacuum for $4 \mathrm{~h}$, sealed under a rubber septum, and then purged with argon for $20 \mathrm{~min}$. Benzyl alcohol (required amount) was added using a syringe. The polymerization reaction was performed by heating the vessel at $130{ }^{0} \mathrm{C}$ for $12 \mathrm{~h}$ under argon atmosphere, with magnetic stirring. The resulting polymer was dissolved in dichloromethane, precipitated with a 10-fold excess of hexane, and dried under reduced pressure (1 torr) for $48 \mathrm{~h}$.

The weight-average molecular weights produced spanned the range from well below $10^{4}$ to over $10^{6}$, and the range of enantiomer proportions was from 100:0 to 50:50. Identification and specifications of the polymers studied are given in Table I.

The room-temperature density of nonvitreous amorphous PLA is estimated by extrapolating a fit to the pooled data of Witzke[Witzke, 1997 \#546 ] on densities above $T_{\mathrm{g}}$, viz.:

$$
\rho(t)=\rho(0) \exp (\alpha t)=1.283_{6} \cdot \exp \left[-7.7\left(10^{-4}\right) t\right] \mathrm{g} / \mathrm{cm}^{3} \text { for } t \text { in }{ }^{\circ} \mathrm{C} \text {. }
$$

Thus

$$
\rho\left(23^{\circ} \mathrm{C}\right)=1.261 \text { and } \rho\left(30{ }^{\circ} \mathrm{C}\right)=1.254 \mathrm{~g} / \mathrm{cm}^{3} \text {. }
$$

Refractive indexes were determined over the wavelength range 300-1300 nm for solid PLAs at room temperature using a variable-angle spectroscopic ellipsometer $\left(\mathrm{VASE}^{\circledR}, \mathrm{J}^{\mathrm{A}}\right.$ A. Woollam Co., Inc., Lincoln, NE.) Samples made with comonomer ratios of 100:0, 80:20, and 50:50 were spin-cast onto silicon wafer substrates to give thicknesses of approximately 150 or $450 \mathrm{~nm}$. The solvent was dichloromethane for the first optical composition and THF for the latter two. Ellipsometric measurements were made after annealing the cast films overnight at $40{ }^{\circ} \mathrm{C}$. 
Thicknesses and optical constants were determined simultaneously using modeling and fitting software (WVASE32 ${ }^{\mathrm{TM}}$ ) provided by the instrument manufacturer (J.A. Woolham, Lincoln, NB USA). Thicknesses were also verified for thicker films using a Tencor P-10 surface profilometer. It was found that the refractive indexes for all compositions could be represented to within experimental error by a two-term Cauchy expression:

$$
n(\lambda)=(1.445 \pm 0.00075)+(4892 \pm 143) \mathrm{nm}^{2} / \lambda^{2} \text { for } 300 \mathrm{~nm}<\lambda<1300 \mathrm{~nm} \text {. }
$$

At the wavelength of the sodium D line, to which most tabulated solvent refractive indexes refer, evaluation of Eqn. 2 gives

$$
n_{\mathrm{PLA}}(589.2)=1.459
$$

Densities $(\rho)$ and refractive indexes $(n)$ for various solvents were taken from standard tables.[Elias, 1989 \#728; Elias, 1989 \#729; Fleischer, 1989 \#730; Sigma-Aldrich, 2002 \#754] The refractive index of a $80 \% / 20 \% \mathrm{w} / \mathrm{w}$ acetonitrile-dichloromethane mixed solvent according to classical[Partington, $1953 \# 740]$ volume additivity of polarizabilities, viz.:

$$
\frac{n^{2}-1}{n^{2}+2}=\sum_{i} \phi_{i} \frac{n_{i}^{2}-1}{n_{i}^{2}+2}
$$

where the $\phi_{\mathrm{i}}^{\prime}$ 's are volume fractions such that

$$
\sum_{i} \phi_{i}=1
$$

With values of the constant densities (for $\mathrm{CH}_{3} \mathrm{CN}, \rho=0.786$, for $\mathrm{CHCl}_{3}, \rho=1.492$ ) and refractive indicies (for $\mathrm{CH}_{3} \mathrm{CN}, n=1.344$, for $\mathrm{CHCl}_{3}, n=1.446$ ), Eqn. 3 predicts the value $n=1.354$ for the mixture. As shown in Figure 2, replicate measurements (at $30^{\circ} \mathrm{C}$ ) of the refractive index of this mixture using an Abbe refractometer (Bausch \& Lomb) produced the same value, 1.354 (cf. Fig. 3). Similarly, the refractive index increment $(\mathrm{d} n / \mathrm{d} c)$ values were computed from the appropriate 
numerical derivatives of solution refractive indexes implied by Equation 3 for low concentrations of PLAs with properties given by Equations 1 and 2. Referring again to Figure 2, direct experimental measurement of PLA solution refractive indexes at various concentrations confirmed our calculated value $(0.08)$ of the slope $\mathrm{d} n / \mathrm{d} c$. Previous PLA studies have not used rigorously cross-checked values for the refractive index increments and many of the literature values are erroneous - this is source of the difficulties represented in Figure 1. A molecular weight determined with an inaccurate $\mathrm{d} n / \mathrm{d} c$ value will be in error by a factor that is the square of the ratio of accurate and inaccurate $\mathrm{d} n / \mathrm{d} c$ values.

Molecular-mass distributions of the polymers of Table I were characterized using sizeexclusion chromatography on Hewlett-Packard (now Agilent Technologies, Palo Alto, CA) PLgel 5- $\mu \mathrm{m}$ mixed-bed columns. Elutions were carried out at ambient temperature with THF as solvent at a flow rate of $1 \mathrm{~mL} / \mathrm{min}$. The light-scattering detector was a triple-angle flow-through instrument (miniDAWN ${ }^{\circledR}$, Wyatt Technology Corp., Santa Barbara, CA) operated with dataacquisition and -analysis software $\left(\right.$ ASTRA $^{\circledR}$ ) provided by the manufacturer. The refractive index increment for PLA in THF, determined using Eqn. 3, was taken as $0.042 \mathrm{~mL} / \mathrm{g}$. The homopolymers $(100 \% \mathrm{~L})$ could not be characterized with SEC because of their insolubility.

Intrinsic viscosities were determined using three different solvents: chloroform, tetrahydrofuran (THF), and an 80/20 w/w mixture of acetonitrile and dichloromethane. Efflux times for thermostated fixed volumes of PLA solutions of varying concentrations in the three solvents were measured (via stopwatch) using Cannon-Übbelohde glass-capillary viscometers. (Cf. ASTM Standard Method D 2857 and conventional references.[Mark, 1945 \#509; Cannon, 1960 \#716; Wagner, $1978 \# 713])$ 
Intrinsic viscosities $[\eta]$ were obtained by global nonlinear least-squares fitting of time-vs.concentration data with the form implied for the experimental domain by the Schulz-Blaschke model:

$$
\frac{\eta}{\eta_{0}}=1+c[\eta] /\left(1-k_{\mathrm{SB}}[\eta] c\right)=\frac{\tilde{t}}{\hat{t}_{0}} \simeq \frac{t}{t_{0}}
$$

or

$$
\tilde{t}=\tilde{t}_{0}\left\{1+c[\eta] /\left(1-k_{\mathrm{SB}}[\eta] c\right)\right\},
$$

where the overtildes denote correction of the observed solvent and solution flow times, $t_{0}$ and $t$ respectively, for kinetic energy and density effects according to

$$
\tilde{t}_{0}=t_{0}\left(1-t_{0}^{-3} E / C\right) \text { and } \stackrel{\tilde{t}}{=} t\left(1-t^{-3} E / C\right)\left[1-c\left(\rho_{\text {polymer }}^{-1}-\rho_{\text {solvent }}^{-1}\right)\right] .
$$

Here $C$ and $E$ are the viscometer calibration and kinetic-energy correction constants as described by Cannon, Manning, and Bell.[Cannon, 1960 \#716]

Figure 3 shows one set of data consisting of corrected efflux times vs. concentrations, along with the global nonlinear fit with Equation 5a. The approach of fitting a multisample and multiconcentration data set to estimate one material-specific concentration factor $k_{\mathrm{SB}}$ and an intrinsic viscosity $[\eta]$ for each solute, has at least three advantages over traditional Huggins or Kraemer extrapolations: (1) It sidesteps some adverse statistical problems of data weighting (especially over-weighting at very low concentrations where effects of polymer sorption on the viscometer walls can become important). (2) Statistics obtainable from fitting include estimates of uncertainties, not only in the flow-time measurements, but also in $k_{\mathrm{SB}}$ and the [ $\eta$ ]'s. (3) Once $k_{\mathrm{SB}}$ has been determined and shown to adequately represent data for a wide range of molecular weights, it can be used as a basis for subsequent single-point determinations of $[\eta]$, since Equation 5 can be solved for $[\eta]$ : 


$$
[\eta]=\frac{\eta / \eta_{0}-1}{c\left[1+k_{\mathrm{SB}}\left(\eta / \eta_{0}-1\right)\right]}=\frac{\hat{t} / \tilde{t}_{0}-1}{c\left[1+k_{\mathrm{SB}}\left(\hat{t} / \hat{t}_{0}-1\right)\right]}
$$

To procure a consistent set of data on molecular weights, radii of gyration, second virial coefficients, and intrinsic viscosities all in a single solvent for the polymers of Table I, we did light-scattering and solution-viscometry experiments using a mixed solvent comprised of acetononitrile and dichloromethane in proportions of 80:20 by weight. The $\mathrm{CH}_{3} \mathrm{CN}$ was selected for its low refractive index (to give fairly high contrast for light scattering: $\mathrm{d} n / \mathrm{d} c=0.08 \mathrm{~mL} / \mathrm{g}$ ), and the $\mathrm{CH}_{2} \mathrm{Cl}_{2}$ was added to increase solvency for PLA so that homopolymers could be included in the measurements. The mixed-solvent light-scattering measurements[Wyatt, 1993 \#734] were made with a Wyatt 18 -angle scattering photometer $\left(\mathrm{DAWN}^{\circledR}\right)$ in batch mode. Multiangle and multiconcentration data were collected and analyzed with software (AURORA ${ }^{\circledR}$ ) provided by Wyatt.

The indispensable experimental quantity needed to specify unperturbed polymer chain dimensions in dilute solutions is the front-factor $K_{\Theta}$ for the theta-condition Mark-Houwink equation,

$$
[\eta]_{\Theta}=K_{\Theta} M^{1 / 2}
$$

Horsky and Bohdanecky recently [Horsky, 1998 \#592] observed, “The method used most frequently for the determination of $K_{\Theta}$ from viscosity data in a good solvent is that of Stockmayer and Fixman in which $K_{\Theta}$ is identified with the intercept of the $[\eta] / M^{1 / 2}$ vs. $M^{1 / 2}$ plot." Much earlier, Billmeyer's textbook[Billmeyer, 1971 \#216 (p 89)] stated, "The best and simplest of these techniques appears to be that of Stockmayer [Stockmayer, 1963 \#757] (1963) ..." Selected examples of standard polymer literature sources that have successfully relied on 
the Stockmayer-Fixman approach are the polyolefin studies of Arnett and Stacy[Arnett, 1973 $\# 162]$ and the critical review of solution chain dimensions and second virial coefficients by Fetters et al.[Fetters, 1994 \#589] In the PLA literature, however, Stockmayer-Fixman extrapolations are not common: Of the several usual references[Tonelli, $1969 \# 571$; Schindler, 1979 \#591; Kim, 1993 \#590; Joziasse, 1996 \#575] that report $K_{\Theta}$ (or, equivalently, $C_{\infty}$ ) values, only Schindler and Harper[Schindler, 1979 \#591] used Stockmayer-Fixman extrapolations. (Unfortunately, the $K_{\Theta}$ values and MH constants reported by Schindler and Harper are in gross disagreement with newer results from solutions, melt rheology, and theoretical simulations and should be disregarded.)

In order to introduce consistency into determinations of $K_{\Theta}$ and $C_{\infty}$ for PLAs, a statistically sound protocol for analyzing data on molecular weights and intrinsic viscosities was implemented. The Stockmayer-Fixman equation, written in the form directly representing what are called "Stockmayer-Fixman" plots ([ $\eta] / M^{1 / 2}$ vs. $\left.M^{1 / 2}\right)$ in the literature, appears as

$$
\frac{[\eta]}{M^{1 / 2}}=K_{\Theta}+b M^{1 / 2}
$$

The slope $b$ is solvent-dependent. The intercept $K_{\Theta}$ can depend slightly on temperature, but should not depend upon the solvent and should certainly never imply the variety shown in Figure 1. Solved for $M$, Equation 8 may be written as

$$
\log M=\log \left(\frac{K_{\Theta}^{2}+2 b[\eta]-K_{\Theta} \sqrt{K_{\Theta}^{2}+4 b[\eta]}}{2 b^{2}}\right) .
$$

Fitting data with this form using nonlinear least squares yields estimates of the parameter values $K_{\Theta}$ and $b$ and their uncertainties. It bears emphasis that Equation $8 \mathrm{a}$ is deliberately cast such that the nonlinear regression problem appears with $\log M$ as the dependent variable and $[\eta]$ as the 
independent variable because the latter is the measured quantity with by far the smaller experimental uncertainty. Spurious effects on least-squares fitting parameter values that result when there is appreciable error in the independent $(x)$ variable can occur. [York, $1966 \# 188$ ] These effects are avoided by consistently fitting with intrinsic viscosity, in which measurement uncertainties are essentially negligible (cf. Table I), as $x$, both in Equation 8a and in the analogous arrangement of the MH equation: $\log M=a^{-1} \log ([\eta] / \mathrm{K})$.

The characteristic ratio is obtained from the value of $K_{\Theta}$ and three constants according to

$$
C_{\infty}=\left(K_{\Theta} / \Phi\right)^{2 / 3} \cdot M_{1} / \overline{L^{2}}
$$

Equation 9 is easily derived by combining definitions in reference [Kratochvil, 1989 \#48]. The three constants are: the mean-squared backbone bond length

$$
\begin{aligned}
\overline{L^{2}} & =\left(L_{\mathrm{C}-\mathrm{C}}^{2}+L_{\mathrm{C}\left(\mathrm{sp}^{2}\right)-\mathrm{O}}^{2}+L_{\mathrm{O}-\mathrm{C}\left(\mathrm{sp}^{3}\right) / 3}^{2}\right) / 3 \\
& =\left(1.527^{2}+1.310^{2}+1.455^{2}\right) / 3 \\
& =2.05 \AA^{2}=2.05\left(10^{-16}\right) \mathrm{cm}^{2},
\end{aligned}
$$

the relative molecular mass per backbone bond

$$
M_{1}=24.02 \text { (for PLA), }
$$

and the "Flory constant"

$$
\Phi=2.55\left(10^{23}\right)
$$

For the right-hand side of Equation 12 we adopt the experimental value (in units consistent with $K_{\Theta}$ in $\mathrm{mL} / \mathrm{g}$ ) of Miyaki et al.[Miyaki, 1980 \#717] recommended by Fujita.[Fujita, 1988 \#718] The bond lengths in Equation 10 are taken from Figure 1a of reference [Alemán, 2001 \#738].

Two variants of the Stockmayer-Fixman method of inferring $K_{\Theta}$ from data on molecular weights and intrinsic viscosities in good solvents are those known as "Tanaka"[Tanaka, 1982 
\#736] and "Dondos-Benoit”[Dondos, 1971 \#727; Horsky, 1998 \#592] plots. In trials of these variants applied to our data, no advantage (nor significantly different results) over use of Equation 8a was found. A detailed analysis of how the Stockmayer-Fixman and Dondos-Benoit plots compare is given in reference $\sim[$ Horsky, 1998 \#592].

Plateau-modulus data can also be used to infer characteristic ratios according to the packing model. In a complementary rheology study to be reported in detail elsewhere, consistent values for plateau moduli[Dealy, 1995 \#58] in PLA melts are obtained by numerically integrating both relaxation spectra and loss modulus[Dealy, 1995 \#58] curves, as prescribed by Ferry[Ferry, 1980 \#46 (pp 373-375)]:

$$
G_{\mathrm{N}}^{0}=\int_{\ln \tau_{\min }}^{\ln \lambda} H(\tau) \mathrm{d}(\ln \tau)=\frac{2}{\pi} \int_{-\ln \lambda}^{-\ln \tau_{\min }} G^{\prime \prime}(\omega) \mathrm{d}(\ln \omega)
$$

where, in the limits of integration, $\ln \tau_{\min }$ is the abscissa where the relaxation spectrum function $H$ has its minimum value in the transition zone and $\lambda$ is a longest relaxation time, beyond which $H(\tau)=0$. Equation 4 of reference $\sim[$ Fetters, $1999 \# 473]$ gives the entanglement molecular weight as

$$
M_{\mathrm{e}}=\rho R T / G_{\mathrm{N}}^{0}
$$

and eq $17 \mathrm{~b}$ of the same reference then gives the characteristic ratio in terms of $M_{\mathrm{e}}$ :

$$
C_{\infty}=10 \rho^{-2 / 3}\left(M_{\mathrm{e}}\right)^{-1 / 3} M_{1} / \overline{L^{2}}
$$

Additional characteristics of chains in the melt that are available from the packing scheme[Fetters, 1996 \#360] are the packing length

$$
P=\frac{M}{\left\langle r^{2}\right\rangle_{0} \rho N_{\mathrm{A}}}
$$

the tube diameter 


$$
d_{\mathrm{t}}=\left(\frac{M_{\mathrm{e}}\left\langle r^{2}\right\rangle_{0}}{M}\right)^{1 / 2}
$$

and the critical entanglement molecular weight[Fetters, 1999 \#475 (eq 7)]

$$
M_{\mathrm{c}}=4.24 \bar{p}^{0.65} M_{\mathrm{e}} .
$$

Finally, theoretical (RIS) methods have also been used to estimate PLA characteristic ratios. The most recent study of which we are aware is that of Blomqvist.[Blomqvist, 2001 \#703]

\section{Results and Discussion}

Tables I and III and Figures 5-7 summarize our main experimental results. In all but two instances, weight-average molecular weights determined by batch light scattering in the mixed solvent agreed, to within their standard uncertainties reported by the Wyatt software, with the corresponding $M_{\mathrm{w}}$ values determined in THF by SEC with light-scattering detection. Because the SEC data include $M_{\mathrm{w}} / M_{\mathrm{n}}$ (and by implication $M_{\mathrm{w}} / M_{\mathrm{v}}$ ) information, we report the SEC-LS values throughout, except in the homopolymer cases, for which Table I lists molecular weights determined in the mixed solvent.

The error limits listed in Table I are approximate standard relative uncertainties in the intrinsic viscosities, estimated from the goodness-of-fit statistics internal to the concentrationflow time data set for each solvent. Total uncertainties will be somewhat larger: For chloroform and THF we suppose the uncertainties might be comparable with those estimated by NIST (approximately $0.9 \%$ ) to apply to certified values of intrinsic viscosities of Standard Reference Materials.[Wagner, 1978 \#713; Guttman, 2001 \#735] In a previous unpublished study using the method of analysis described here, one of us (JJ) obtained an intrinsic viscosity value for SRM 1475 that agreed with the certified value to within about $0.7 \%$. Accordingly, the methods we 
employed have been found to produce at least one result in full agreement with a value certified by NIST for a Standard Reference Material, a polyethylene.

Figure 5 shows the Mark-Houwink fit for relating $M_{\mathrm{w}}$ of PLAs to intrinsic viscosities in chloroform. Close analysis of the data of Figure 5 reveals no consistent effect of comonomer content upon the molecular weight-intrinsic viscosity relationship. The same conclusion was similarly reached for the other solvents, as well.

Figure 6 is for the same data less the homopolymers. Here the MH fit gives slightly different constants because polymolecularity corrections were applied to obtain ordinates in terms of viscosity averages, $M_{\mathrm{v}}$. This figure also shows results of fitting the same data with the Stockmayer-Fixman form, eq 8a. Results obtained in this manner for all three solvents are collected in Table III. As the solvents vary, good agreement among the determined $K_{\Theta}$ values and a systematic decrease of the MH $a$ parameter with decreasing solvency are found.

Figure 7 is a plot in the Stockmayer-Fixman format (eq 8), reconstructed from the constants in Table III. This is for contrast with Figure 1: The intercepts are within experimental error the same, though the slopes, as expected, increase systematically in the order of the goodness of the solvents as indicated by the MH exponents.

Figure 8 plots second virial coefficients determined from our mixed-solvent light scattering data using the Berry plot[, $1996 \# 758]$ method of analysis. For comparison, a curve depicting the Orofino-Flory relationship (eq 13) is also shown. Agreement between the curve and the data is obviously poor. This is not an indictment of the data, however, because the results show, at least semiquantitatively, the same behavior as in Figure A1 of the appendix for the well- 
documented polystyrene-benzene system. Rather, these results indicate that attempting to determine $K_{\Theta}$ using equations $13 \mathrm{a}$ and $14 \mathrm{a}$ is ill advised.

Figure 9 indicates that the mixed-solvent data on molecular weights, intrinsic viscosities, and molecular sizes expressed as root-mean-squared radii of gyration $(s)$ are in general internally consistent: Experimental $s$ values are plotted against estimated $M_{\mathrm{z}}$ 's and compared with predictions from the well-grounded Flory-Fox[Flory, 1951 \#608] equation in the form

$$
s=\left\langle s^{2}\right\rangle^{1 / 2}=\left(\frac{[\eta] M}{6^{3 / 2} \Phi}\right)^{1 / 3}
$$

Adequate over-all agreement is observed, although the experimental $s$ values are too scattered to be useful for more detailed analysis.

Figure 10 shows an example of a dynamic storage modulus master curve for a PLA melt, along with the plateau modulus value, determined using eq 15, clearly marked at $1 \mathrm{MPa}$. Utilizing this result within the packing model provides additional insight into properties of PLA. In Table IV are collected various PLA melt characteristics obtained using equations 16-20. For comparison, values determined similarly for three other typical linear flexible polymers are also tabulated. Not surprisingly, the number of backbone bonds between entanglements in PLA is somewhat greater than in poly(ethylene oxide) (PEO), which has the same (C-C-O-) backbone but lacks pendant oxygen and methyl groups. Correspondingly, $M_{\mathrm{c}} / M_{1}$ is also somewhat greater for PLA than for PEO. Analogous comparisons are shown between isotactic polypropylene and polyethylene, another pair having the same (C-C-) backbone but differing by pendant methyls. Notice that, as has recently been extensively discussed by Heymans,[Heymans, 2000 \#471] characteristic ratios alone do not necessarily directly indicate comparative chain stiffnesses. 


\section{Conclusions}

It is surprising that the state of commercial development of PLA has advanced so far while literature reports of scientifically well-defined fundamental properties of the material have remained so confusing. Much of the difficulty with the previous literature evidently stems from inaccuracies in what should be fairly trivial determinations of specific refractive index increments. This seems egregious, given that the additivity rule (eq 3) for polarizabilities is a thoroughly tested principle of 19th-Century chemical physics.[Partington, 1953 \#740] Nonetheless, scrutiny of the literature reveals prevalent use of implausible $\mathrm{d} n / \mathrm{d} c$ values. In this study, mutually confirming experimental and theoretical determinations of refractive indexes of the polymers, solvents, and solutions show that refractive index increments used in earlier published light-scattering determinations of PLA molecular weights are often suspect. This circumstance is largely responsible for discrepancies between characteristic ratios reported here and others found in the literature. Reliance on the Orofino-Flory equation for inferring characteristic ratios from second virial coefficients has been another factor contributing to disparities in reported characteristic ratios.

Values of $C_{\infty}$ determined by solution, melt rheology, and simulation agree and imply that the polylactides are typical linear flexible polymers. Stockmayer-Fixman fitting of intrinsic viscosity data for three different solvents yields a consistent Mark-Houwink scale factor for theta conditions: $K_{\Theta}=0.107 \pm 0.022 \mathrm{~mL} / \mathrm{g}$. This corresponds to unperturbed chain dimensions described by a characteristic ratio $C_{\infty}=6.5 \pm 0.9$. Melt properties are consistent with the packing model propounded by Fetters and co-workers. Using this scheme and an experimentally

measured plateau modulus $G_{\mathrm{N}}{ }^{0}=1.0 \mathrm{MPa} \pm 20 \%$ leads to an implied $C_{\infty}$ in the range $6.7 \pm 0.7$. Recently published theoretical simulation results[Blomqvist, 2001 \#703] may be summarized as 
indicating characteristic ratios in the range $6.1 \pm 1.3$, in excellent agreement with our experimental results. While Blomqvist's computational study does find a systematic trend with optical composition, the predicted magnitude of the effect is small and lies within the range of uncertainty of the present careful experimental investigation. Appreciably larger values of $C_{\infty}$ or a strong dependence of this ratio on optical composition is not confirmed either experimentally or theoretically.

Mark-Houwink and Schulz-Blaschke constants for dilute PLA solutions in chloroform and in THF were determined precisely. For chloroform at $30{ }^{\circ} \mathrm{C},[\eta]=0.0131 M_{\mathrm{v}}{ }^{0.777}$ or $[\eta]=$ $0.0153 M_{\mathrm{w}}^{0.759} \mathrm{~mL} / \mathrm{g}$, and $k_{\mathrm{SB}}=0.302$; for THF, $[\eta]=0.0174 M_{\mathrm{v}}^{0.736} \mathrm{~mL} / \mathrm{g}$, and $k_{\mathrm{SB}}=0.289$. These values enable a direct connection between single-point dilute-solution relative viscosity measurements, as practiced industrially, with absolute molecular weights. In addition, they provide a basis for determining absolute molecular mass distributions using the universal calibration[Grubisic, 1967 \#715] technique with size-exclusion chromatographs lacking lightscattering detection.

No influence of optical composition upon solution properties could be discerned, and no hydrodynamic size anomalies attributable to branching were observed. Pooled intrinsic viscosities follow MH power-law relationships with molecular weights that are commonplace for such polymers. Values of the MH parameters are near to averages for other linear flexible polymers in general.[Aharoni, 1977 \#470; Rai, 1997 \#391] Solubilities in various solvents are affected only indirectly by optical composition, specimens containing crystallinity being more difficult to dissolve than amorphous ones, except in chlorinated solvents. We did not obtain any conclusive data on temperature dependence of $\left\langle r^{2}\right\rangle_{0} / M$, either in melts or solutions. 


\section{Acknowledgements}

The authors gratefully acknowledge funding support from the EPA and NSF under the Technologies for a Sustainable Environment program and from the U. S. Department of Energy under the Agricultural Industries of the Future program of the Office of Industrial Technologies. Discussions with Jed Randall of Cargill Dow LLC contributed to the quality of this work

\section{Appendix: Testing the Orofino-Flory Equation}

A crucial test of eq 12 is readily available using, to give only one example, the extensive polystyrene data assembled in the critical review by Fetters et al.[Fetters, 1994 \#589] As the authors of that review remarked, polystyrene systems are probably the most extensively studied and well-documented polymer solutions available, for at least two strong fundamental reasons: (1) Samples spanning a very wide range of molecular weights, with very narrow molecular mass distributions, are available via anionic polymerizations. (2) Cyclohexane is a theta solvent near room temperature, which greatly simplifies experimentation with these systems, and leaves little uncertainty in the derived theta-condition Mark-Houwink front factor, $K_{\Theta}$. The experimental data we cited above as the source[Miyaki, $1980 \# 717$ ] for a preferred[Fujita, 1988 \#718] value of the Flory constant $\Phi$ are for polystyrenes in cyclohexane.

In Figure A1 we plot, against molecular weight, the measured second virial coefficients for polystyrenes in benzene given in Table 8 of reference $\sim$ [Fetters, 1994 \#589]. In the same figure we show the $A_{2}$ predictions of the Orofino-Flory eq 12, with, as inputs, the well-established constant value of $K_{\Theta}=0.0844 \mathrm{~mL} / \mathrm{g}$, together with the good-solvent (benzene) $\mathrm{MH}$ constants from eq 33 of reference $\sim[$ Fetters, $1994 \# 589]$. 
To use experimentally measured second virial coefficient $\left(A_{2}\right)$ values to determine $K_{\Theta}$ with the Orofino-Flory theory, one uses eq 14 rearranged:

$$
K_{\Theta}=\frac{K M^{a-1 / 2}}{\alpha^{3}}
$$

with $\alpha^{3}$ given by eq 13 rearranged as

$$
\alpha^{3}=\left\{1+\frac{2}{\sqrt{\pi}}\left(\exp \left(\frac{27}{8} \frac{\sqrt{2}}{\pi} \frac{\Phi}{N_{\mathrm{A}}} \frac{M}{[\eta]} A_{2}\right)-1\right)\right\}^{3 / 2}
$$

With the polystyrene reference data from Table 8 of Fetters et al.,[Fetters, 1994 \#589] the combination of equations $13 \mathrm{a}$ and $14 \mathrm{a}$ predicts $K_{\Theta}$ values varying smoothly from 0.037 to 0.21 $\mathrm{mL} / \mathrm{g}$ at the low and high ends of the tabulated molecular weight range, respectively. The inference of values varying over this wide range instead of the constant 0.0844 is obviously unsatisfactory. 


\section{Tables}

Table I. Experimental polylactides

\begin{tabular}{|c|c|c|c|c|c|c|}
\hline \multirow[t]{2}{*}{ SH-PLA- } & \multirow[t]{2}{*}{$\% \mathrm{~L} / \% \mathrm{D}$} & \multirow[t]{2}{*}{$10^{-3} M_{\mathrm{n}}$} & \multirow[t]{2}{*}{$10^{-3} M_{\mathrm{w}}$} & \multicolumn{3}{|c|}{$[\eta], \mathrm{mL} / \mathrm{g}\left(30^{\circ} \mathrm{C}\right)$} \\
\hline & & & & $\mathrm{CHCl}_{3}$ & THF & $\begin{array}{c}\mathrm{CH}_{3} \mathrm{CN} \& \\
\mathrm{CH}_{2} \mathrm{Cl}_{2}\end{array}$ \\
\hline 71 & $100 / 0$ & & & $29.50 \pm 0.33 \%$ & & $17.5 \pm 1.2 \%$ \\
\hline 72 & $100 / 0$ & & 131 & $109.5 \pm 0.36 \%$ & & $55.8 \pm 1.3 \%$ \\
\hline 68 & $0 / 100$ & & & $112.1 \pm 0.36 \%$ & & $60.3 \pm 1.2 \%$ \\
\hline 73 & $100 / 0$ & & 276 & $211.4 \pm 0.37 \%$ & & $95.2 \pm 1.3 \%$ \\
\hline 16 & $100 / 0$ & & 1760 & $746.4 \pm 0.51 \%$ & & $331 . \pm 1.3 \%$ \\
\hline 30 & $80 / 20$ & 3.900 & 4.56 & $9.412 \pm 0.32 \%$ & $8.203 \pm 0.32 \%$ & $7.16 \pm 2.4 \%$ \\
\hline 15 & $80 / 20$ & 14.15 & 18.3 & $30.75 \pm 0.33 \%$ & $26.30 \pm 0.32 \%$ & $19.3 \pm 1.3 \%$ \\
\hline 14 & $80 / 20$ & 70.40 & 103 & $99.80 \pm 0.37 \%$ & $81.05 \pm 0.31 \%$ & $55.9 \pm 1.2 \%$ \\
\hline 3 & $80 / 20$ & 132.6 & 188.7 & & $118.3 \pm 0.31 \%$ & \\
\hline 2 & $80 / 20$ & 137 & 213.2 & & $135.6 \pm 0.31 \%$ & \\
\hline 9 & $80 / 20$ & 255 & 433.3 & & $255.1 \pm 0.42 \%$ & \\
\hline 19 & $80 / 20$ & 92.50 & 142 & $130.4 \pm 0.38 \%$ & & $71.7 \pm 1.2 \%$ \\
\hline 31 & $80 / 20$ & 351.8 & 570 & $426.9 \pm 0.37 \%$ & & $173 . \pm 1.8 \%$ \\
\hline 13 & $80 / 20$ & 449.8 & 692.7 & $519.2 \pm 0.38 \%$ & & $267 . \pm 1.5 \%$ \\
\hline 41 & $50 / 50$ & 14.0 & 18.3 & $24.37 \pm 0.33 \%$ & & $16.9 \pm 1.2 \%$ \\
\hline 43 & $50 / 50$ & 84.0 & 172 & $125.5 \pm 0.37 \%$ & & $65.7 \pm 1.2 \%$ \\
\hline 44 & $50 / 50$ & 127 & 245 & $149.0 \pm 0.38 \%$ & & $71.4 \pm 1.3 \%$ \\
\hline 18 & $50 / 50$ & 288.8 & 459 & $251.3 \pm 0.41 \%$ & & $174 . \pm 2.1 \%$ \\
\hline
\end{tabular}


Table II. Dilute-solution constants for PLAs at $30{ }^{\circ} \mathrm{C}$

\begin{tabular}{|c|c|c|c|c|}
\hline \multicolumn{2}{|c|}{ parameters } & \multicolumn{3}{c|}{ solvent } \\
\cline { 3 - 5 } & $k_{\mathrm{SB}}:$ & $0.302 \pm 0.005$ & $0.289 \pm 0.005$ & $0.373 \pm 0.016$ \\
\hline concentration factor & $K, \mathrm{~mL} / \mathrm{g}:$ & $0.0131 \pm 0.0048$ & $0.0174 \pm 0.0046$ & $0.0187 \pm 0.0075$ \\
\hline \multirow{2}{*}{ Mark-Houwink fit } & $a:$ & $0.777 \pm 0.031$ & $0.736 \pm 0.023$ & $0.697 \pm 0.034$ \\
\cline { 2 - 5 } & $K_{\Theta}, \mathrm{mL} / \mathrm{g}:$ & $0.112 \pm 0.017$ & $0.101 \pm 0.014$ & $0.096 \pm 0.011$ \\
\hline \multirow{2}{*}{ Stockmayer-Fixman fit } & $10^{4} b:$ & $5.82 \pm 0.69$ & $4.76 \pm 0.65$ & $2.30 \pm 0.36$ \\
\cline { 2 - 5 } & & $6.74 \pm 0.67$ & $6.29 \pm 0.57$ & $6.08 \pm 0.46$ \\
\hline characteristic ratios & $C_{\infty}$ (from eq 9) : & & & \\
\hline
\end{tabular}


Table III. Flexible linear polymer melt chain dimension comparisons

\begin{tabular}{|l|c|c|c|c|}
\hline \multirow{2}{*}{ parameters } & \multicolumn{4}{|c|}{ values } \\
\cline { 2 - 5 } & PLA $\left(140^{\circ} \mathrm{C}\right)$ & PEO $\left(140^{\circ} \mathrm{C}\right)$ & it-PP $\left(190^{\circ} \mathrm{C}\right)$ & PE $\left(140^{\circ} \mathrm{C}\right)$ \\
\hline $\begin{array}{l}M_{1} \text { (relative molecular mass } \\
\text { per backbone bond) }\end{array}$ & $24.02($ eq 11) & 14.68 & 21.04 & 14.027 \\
\hline$\overline{L^{2}, \AA^{2}}$ & $2.05($ eq 10) & 2.11 & 2.37 & 2.42 \\
\hline$\rho, \mathrm{g} / \mathrm{cm}^{3}$ & $1.152($ eq 1) & 1.034 & 0.759 & 0.792 \\
\hline$G_{\mathrm{N}}{ }^{0}, \mathrm{MPa}$ & $1.0($ Fig. 10$)$ & 1.8 & 0.43 & 2.6 \\
\hline$M_{\mathrm{e}}($ eq 16$)$ & 3,959 & 1,973 & 6,797 & 1,046 \\
\hline$M_{\mathrm{e}} / M_{1}$, backbone bonds & 165 & 134 & 323 & 75 \\
\hline$M_{\mathrm{c}}$ (eq 20) & 9,211 & 5,330 & 13,635 & 3,164 \\
\hline$M_{\mathrm{c}} / M_{1}$, backbone bonds & 383 & 363 & 648 & 226 \\
\hline$<r^{2}>_{0} / M, \AA^{2}$ & 0.574 & 0.805 & 0.694 & 1.25 \\
\hline$C_{\infty}$ (eq 17) & 6.7 & 5.6 & 6.2 & 7.3 \\
\hline$p$ (packing length, eq 18), $\AA$ & 2.51 & 1.99 & 3.15 & 1.68 \\
\hline$d_{\mathrm{t}}$ (tube diameter, eq 19), $\AA$ & 47.7 & 39.9 & 68.7 & 36.2 \\
\hline$d_{\mathrm{t}} / p$ (Ronca-Lin ratio) & 19.0 & 20.0 & 21.8 & 21.6 \\
\hline
\end{tabular}




\section{Figures}

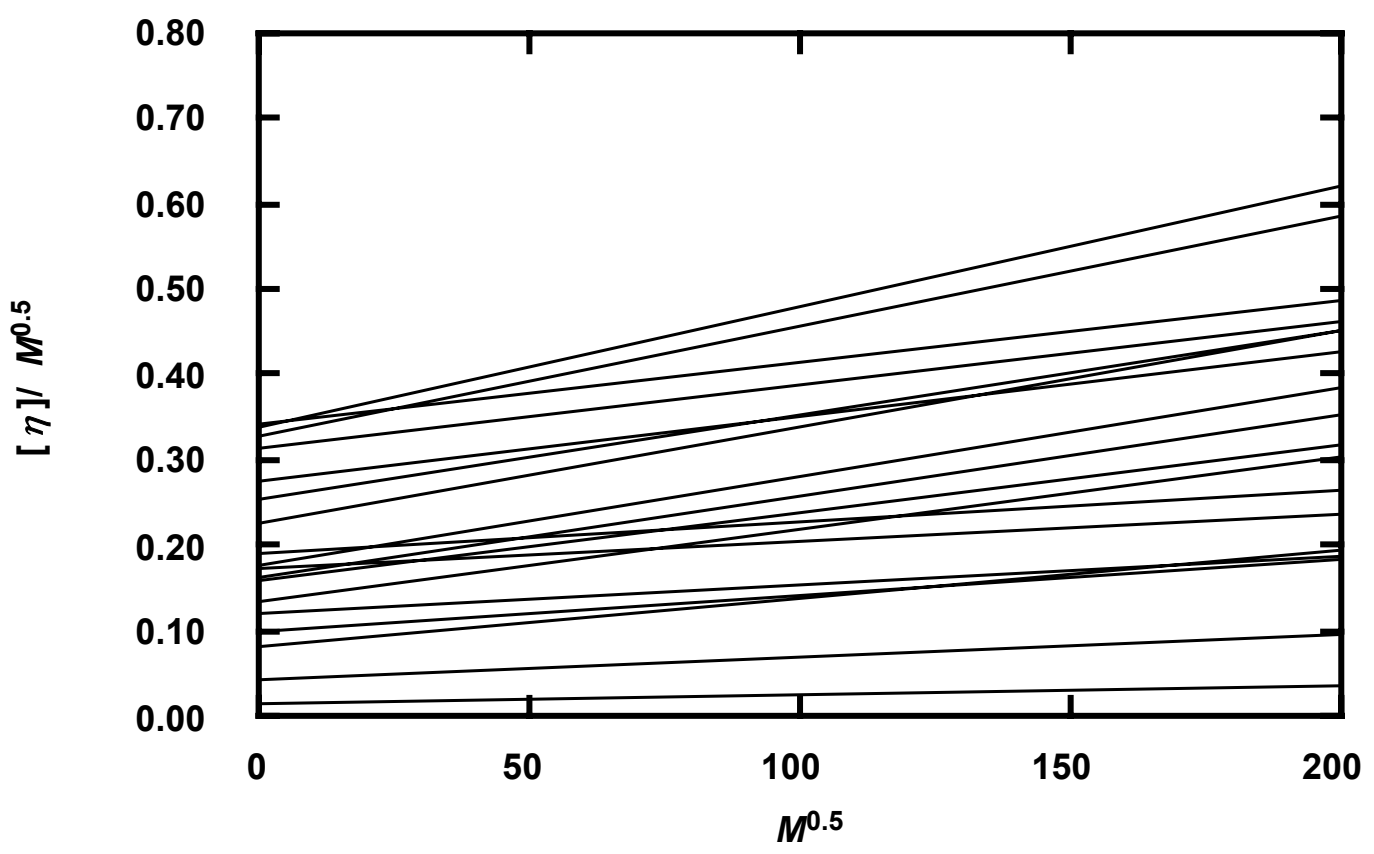

Figure 1. Stockmayer-Fixman plots reconstructed from published $\mathrm{MH}$ parameters, illustrating the confused state of the literature on PLAs. Rather than the wide range of values exhibited, the $y$-axis intercepts should be a common value equal to $K_{\Theta}$. 


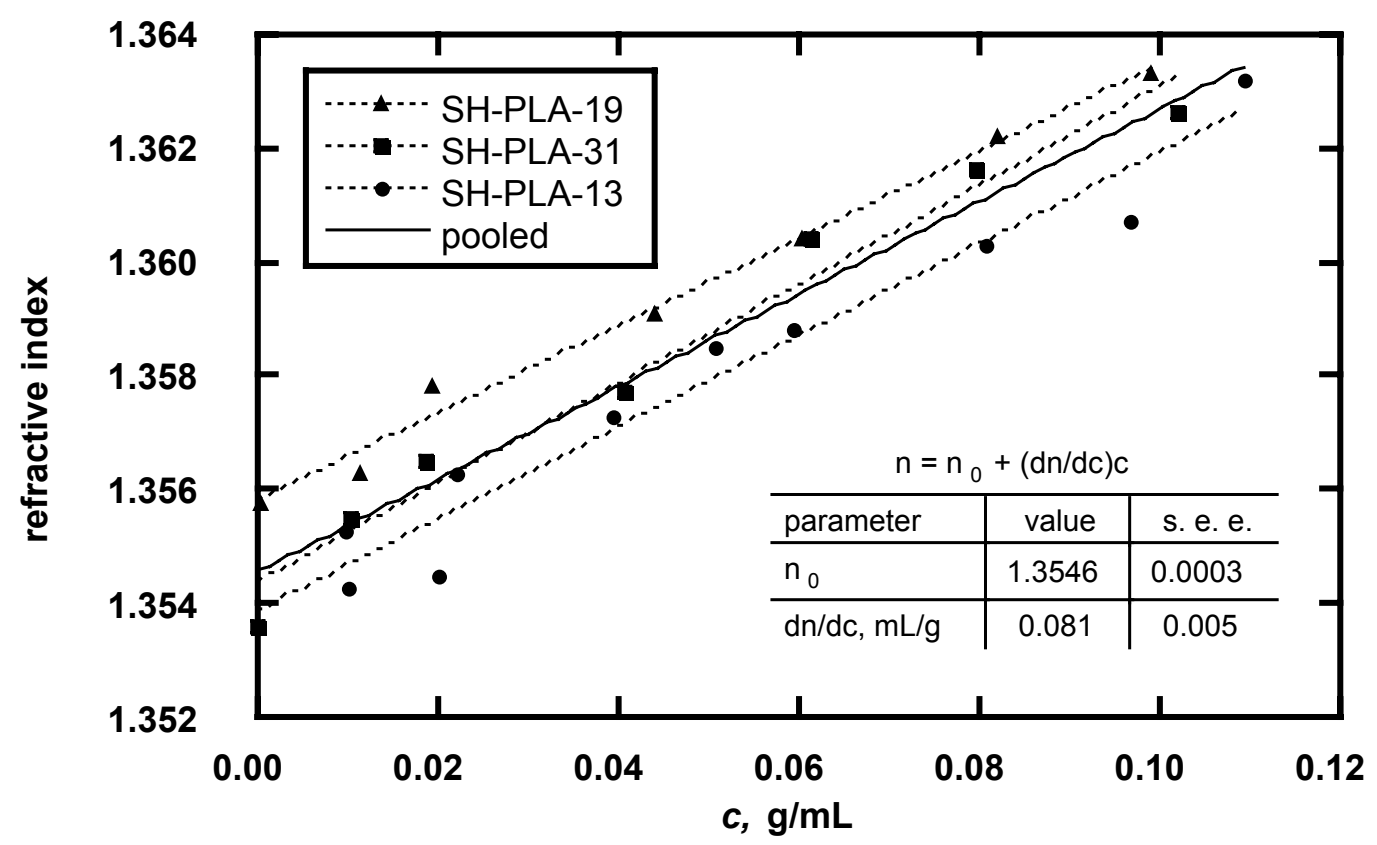

Figure 2. Measured indexes of refraction for PLAs dissolved in an $80 / 20 \mathrm{w} / \mathrm{w}$ mixture of acetonitrile and dichloromethane. The experimentally determined $(\mathrm{d} n / \mathrm{d} c)=0.081 \mathrm{~mL} / \mathrm{g}$ is in complete agreement with that calculated using Eqn. 3. 


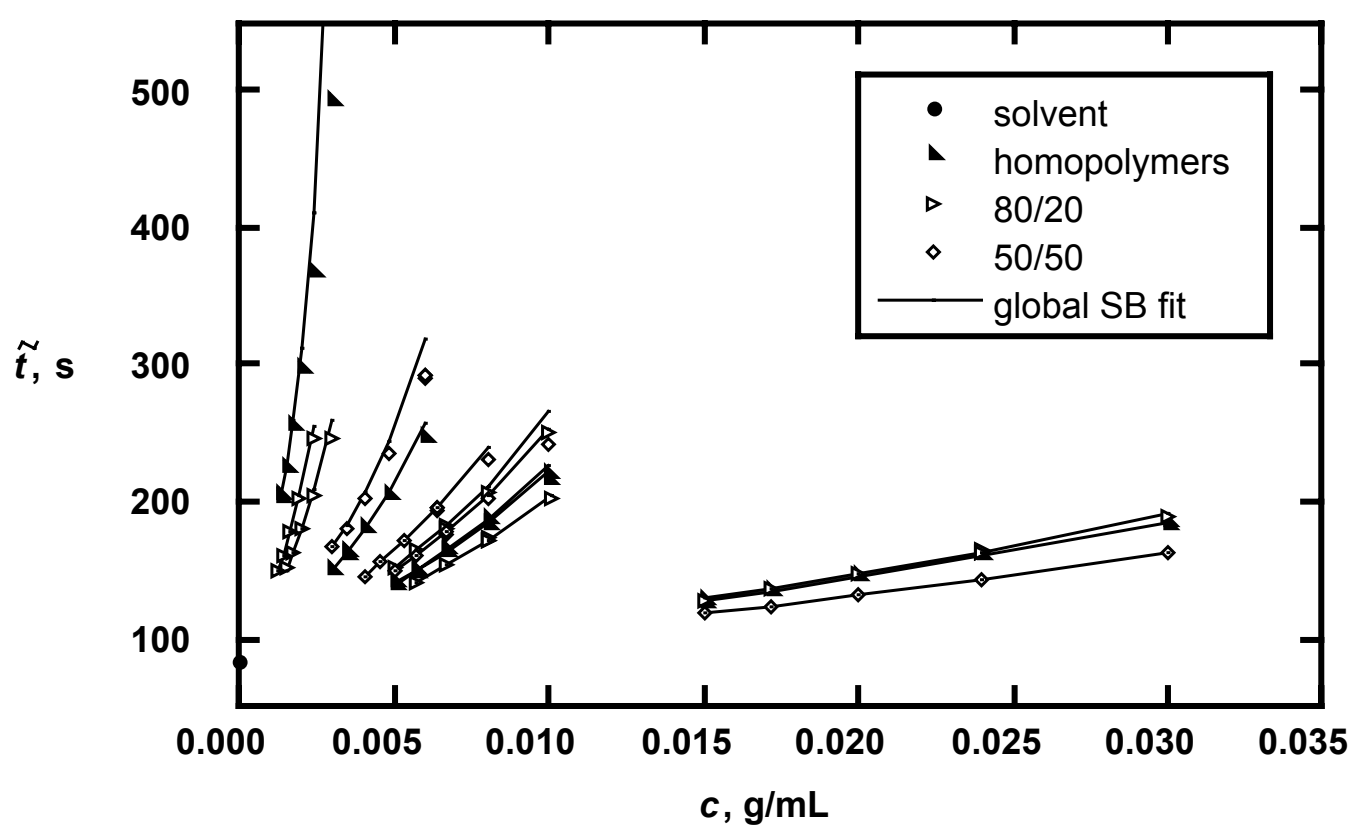

Figure 3. Corrected capillary flow times vs. concentrations for PLAs in chloroform. Curves represent a global fit to the Schultz-Blaschke relationship in the form of eq 5a (see text for details). Points representing relative viscosities greater than 2 were excluded from the fit optimization. 


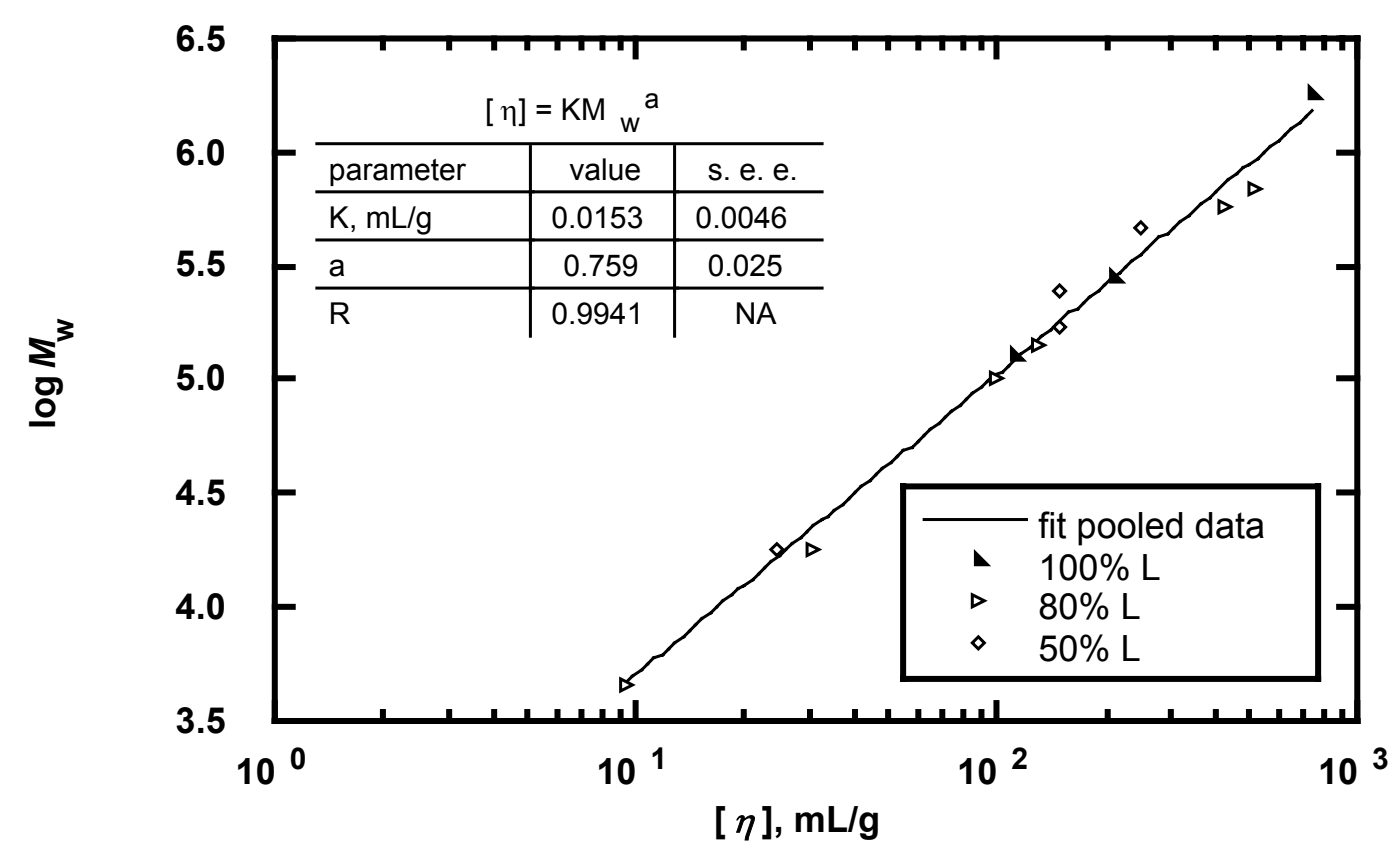

Figure 5. PLA absolute weight-average molecular weights, determined by SEC with lightscattering detection, vs. intrinsic viscosities in chloroform at $30{ }^{\circ} \mathrm{C}$; no systematic effect of optical composition is discernible. 


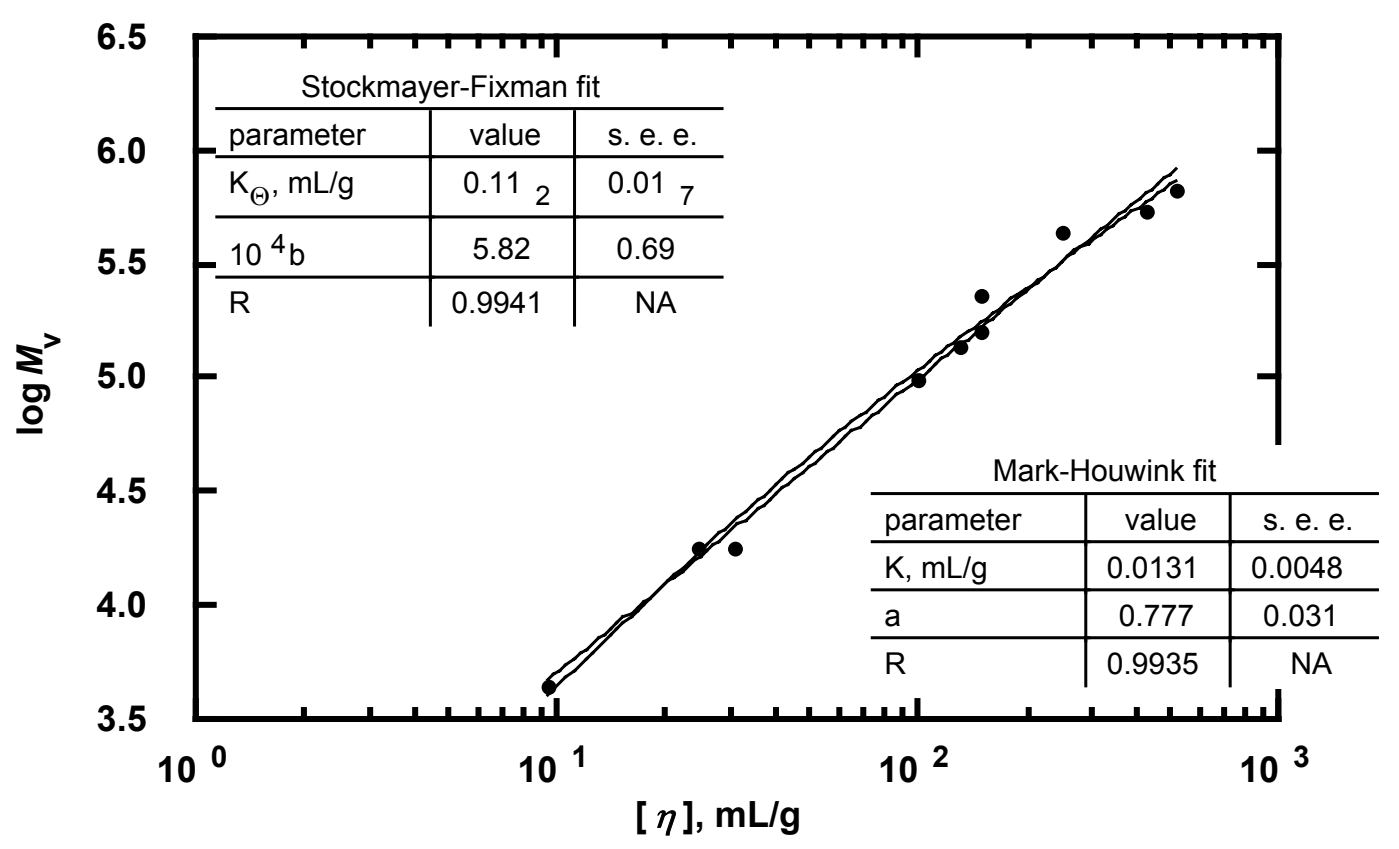

Figure 6. Viscosity-average molecular weights vs. intrinsic viscosity for PLAs in chloroform allowing concurrent determinations of $\mathrm{MH}$ constants and $K_{\Theta}$ (see text for details). The Stockmayer-Fixman fit appears slightly curved in these coordinates. 


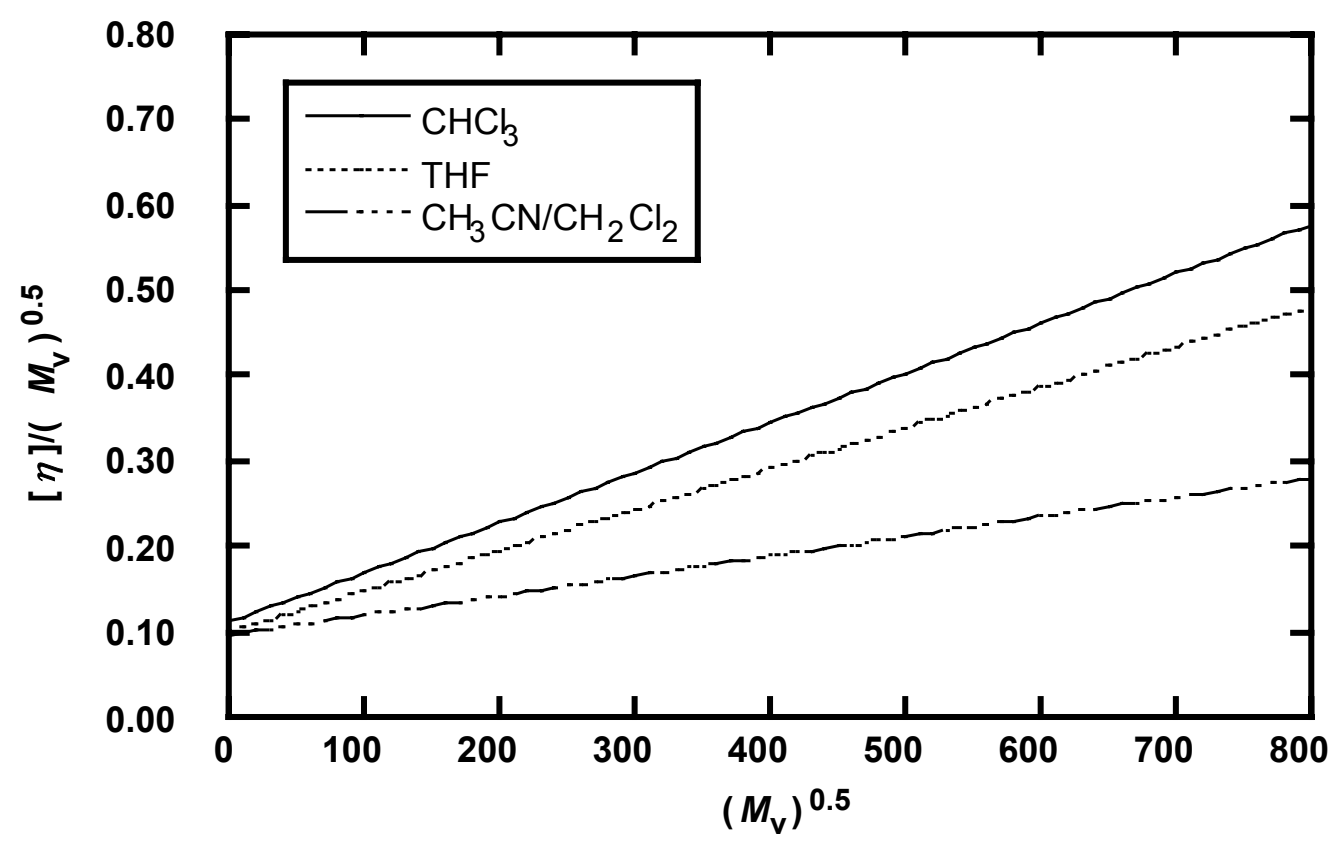

Figure 7. Stockmayer-Fixman plots for three separate solvents used in the present study showing consistency of the determined $K_{\Theta}$ values (contrast with Fig. 1). 


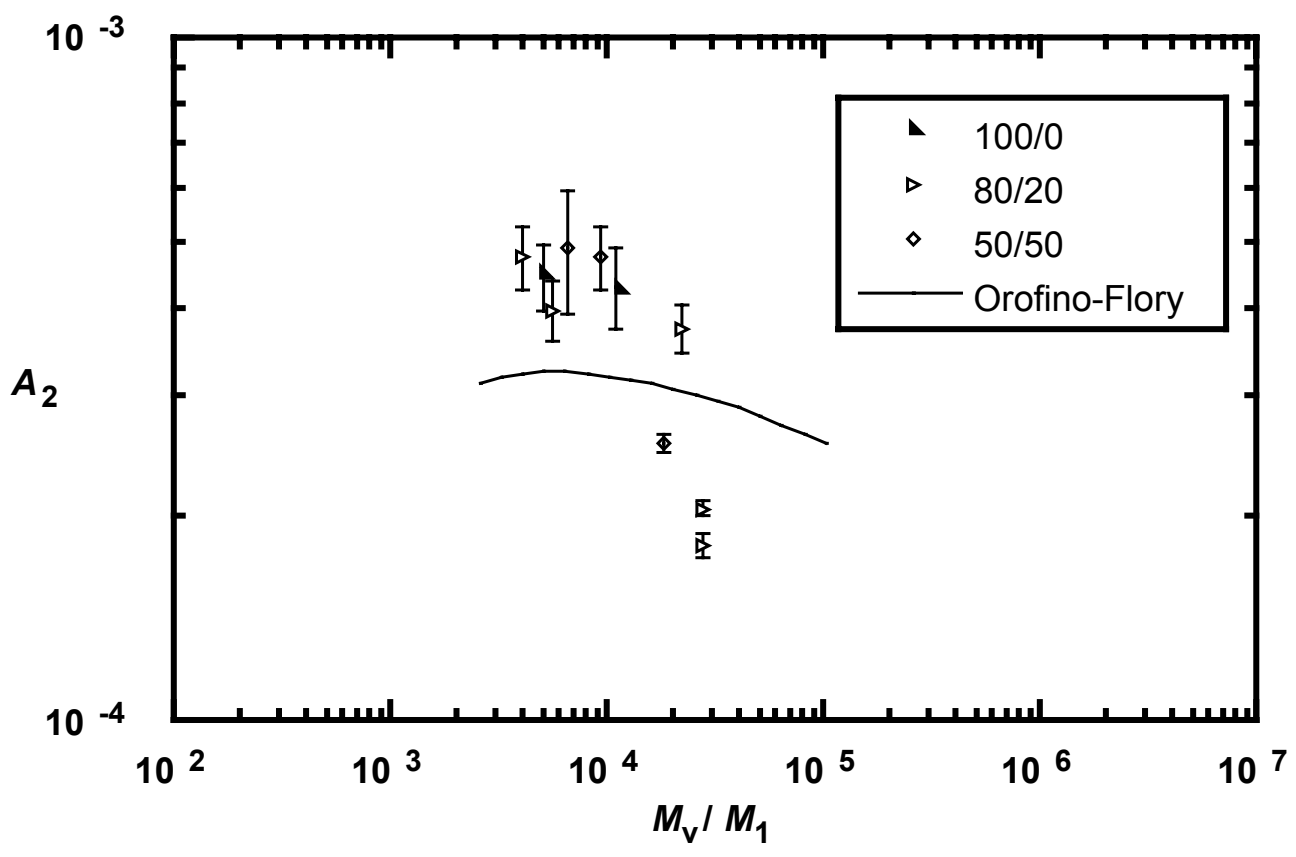

Figure 8. Experimentally determined second virial coefficients vs. chain lengths compared to values obtained from the Orofino-Flory theory as expressed by eq 12 of the text (compare Fig. A1). 


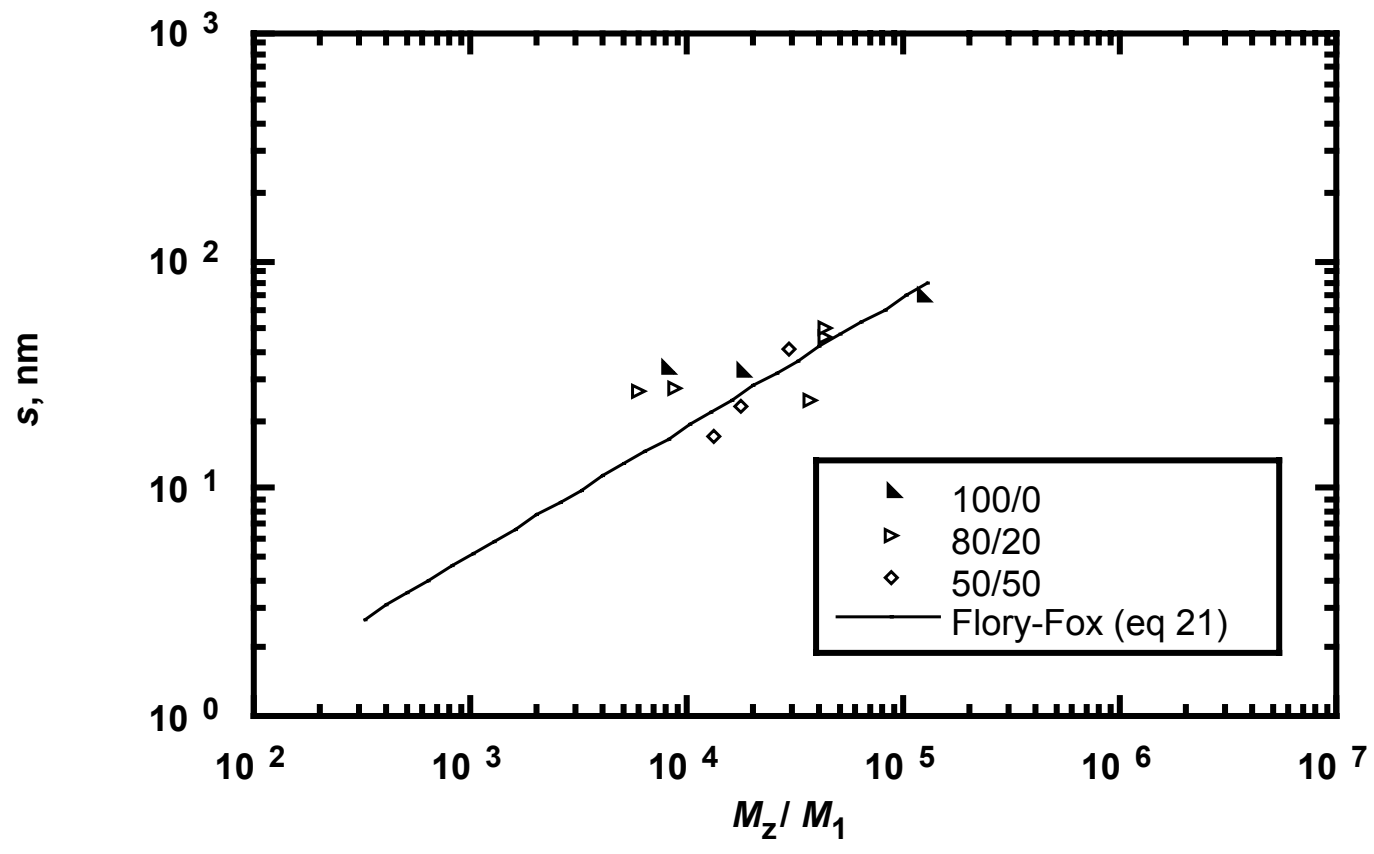

Figure 9. Measured radii of gyration vs. chain lengths compared to the Flory-Fox equation (21 of the text) demonstrating general internal consistency in the static light-scattering data. 


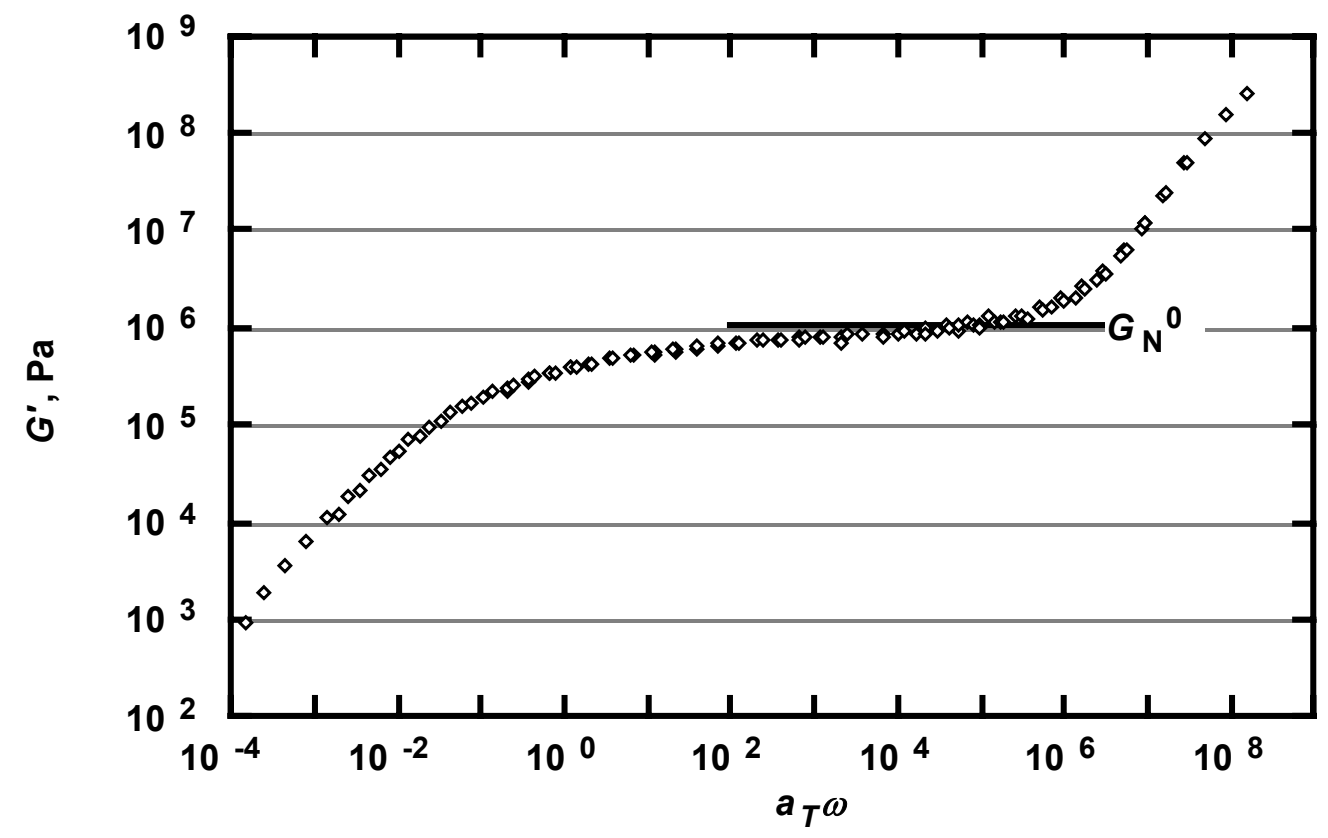

Figure 10. Dynamic storage modulus vs. shifted frequency for a high-molecular-weight amorphous PLA (last sample in Table I) referenced to a temperature of $107^{\circ} \mathrm{C}$. The value of the plateau modulus is near $1 \mathrm{MPa}$, implying single-chain properties consistent with solution measurements. 


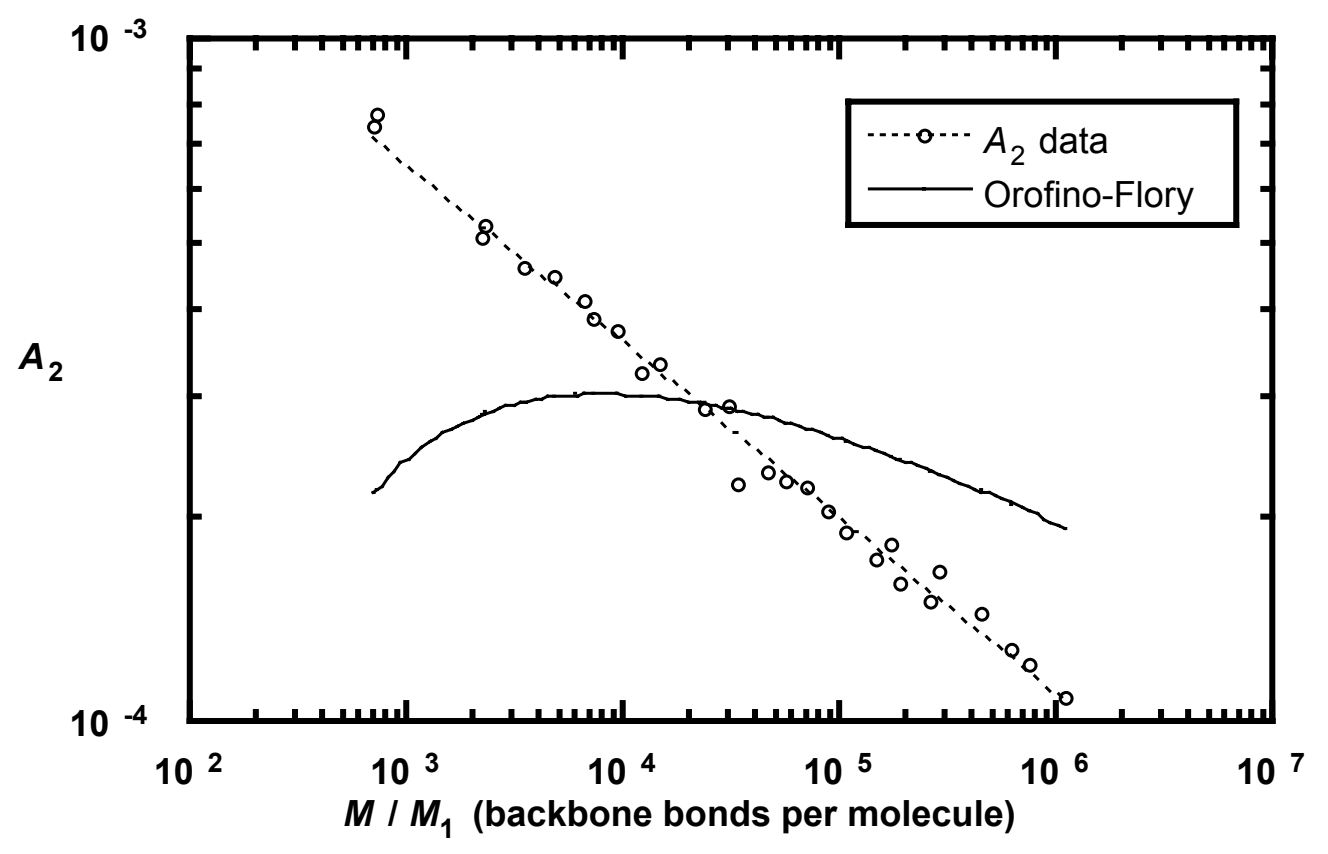

Figure A1. Experimentally determined second virial coefficients vs. chain lengths for the polystyrene-benzene system compared to values obtained from the Orofino-Flory theory (compare Fig. 8). 


\section{References}




\title{
Melt Rheology of Variable L-content Poly(lactic acid)
}

\author{
Jay Janzen, John R. Dorgan, ${ }^{*}$ Michael P. Clayton ${ }^{\diamond}$, Daniel M. Knauss, ${ }^{\dagger}$ and Sukhendu B. Hait, ${ }^{*}$ \\ Chemical Engineering Department and Department of Chemistry and Geochemistry \\ Colorado School of Mines, Golden, Colorado 80401
}

\begin{abstract}
Polylactides (PLAs) have been known for several decades and have recently gained considerable commercial significance. This development makes it urgently desirable to have the rheological properties of these materials well-characterized. However, comprehensive rheological characterization of PLAs is not yet available from the literature. In this study, rheological and thermal measurements were made on a comprehensive and well-characterized set of homopolymers and copolymers spanning wide ranges of molecular mass and stereoisomer proportions (L-content). For the weight average molecular weight range of $10^{5}$ to $10^{6}$ (g/mol) and a reference temperature of $180 \mathrm{C}$, the zero shear viscosity is described well by the relationship $\log \left(\eta_{0}\right)=-14.26+3.4 \log \left(M_{w}\right)$ regardless of the stereochemical composition. Glass transition temperatures do show a trend of increased values with increased L-content; the values of the glass transition temperatures at infinite molecular weight for 100,80 , and $50 \%$ L-content are $60.2{ }^{\circ} \mathrm{C}, 56.4{ }^{\circ} \mathrm{C}$, and $54.6^{\circ} \mathrm{C}$, respectively. The data imply polylactides are typical linear glass-forming polymers. Unperturbed PLA chain dimensions well above their glass transition temperatures are describable in terms of a characteristic ratio in the range $6.5 \pm 0.9$, independent of stereoisomeric composition.
\end{abstract}

\footnotetext{
* Author for correspondence. e-mail: jdorgan@mines.edu

$\checkmark$ Present affiliation: Dept. of Chemical Engineering, Univ. of Queensland

$*$ Dept. of Chemistry and Geochemistry.
} 
Keywords: polylactide or poly(lactic acid), PLA, rheology, physical properties, characteristic ratio, packing model 


\section{Introduction}

Background. Polylactides are environmentally benign and degradable polymers ${ }^{1}$ made from renewable resources. They have physical properties that make them useful for fibers, packaging, and other applications traditionally dominated by petroleum-based resins. The recent advent of large-scale commercial production ${ }^{\mathrm{a}}$ of these materials makes characterization of their fundamental properties urgently desirable. Although the general literature on polylactides is extensive, only a few references ${ }^{2-7}$ have considered rheological properties, and those have not captured a systematic description of PLA rheology across a broad range of stereooptical composition (i.e. across a wide range of L-content).

\section{Materials and Methods}

This study reports findings obtained using differential-scanning calorimetric (DSC) and rheometric measurements made on a set of polylactide (PLA) polymers described and characterized carefully in solution, as described elsewhere. ${ }^{8}$ The materials studied span a combined range of molecular mass and stereoisomeric comonomer composition that is unprecedented in the literature on polylactides.

Homopolymers and copolymers of enantiomeric lactides (cyclic dimers of lactic acid) were synthesized using stannous octanoate-catalyzed ring-opening polymerizations as detailed in reference ${ }^{8}$. Weight-average molecular weights obtained spanned the range from below $10^{4}$ to over $10^{6}$, and the range of enantiomer proportions (L:D) was from 100:0 to 50:50. Identification and specifications of the polymers studied are given in Table I.

\footnotetext{
${ }^{a}$ For details see http://www.cargilldow.com
} 
The principal results to be reported here are from measurements made using an ARES rheometer (TA Instruments/Rheometric Scientific, Piscataway, NJ). Because the 80:20 and 50:50 copolymers do not crystallize, they are amenable to dynamic shear rheometry over a wide range of temperature. Melt temperatures safely below those where degradation causes experimental difficulties are easily accessible and these fully amorphous polymers may be probed down into the glass-transition interval. At the lower temperatures, it is necessary to use small-diameter $(8 \mathrm{~mm})$ parallel plates and very small deformation amplitudes in order to remain within the limits of linear viscoelasticity and to avoid overloading the instrument. A typical schedule for a sequence of isothermal frequency sweeps at decreasing temperatures, after zeroing the gap at $90{ }^{\circ} \mathrm{C}$ and then loading and trimming a specimen at the maximum temperature, 170 ${ }^{\circ} \mathrm{C}$, appears in Table II. This table indicates values that must be separately entered into the commanded gap setting and actual geometry specification menus of the instrument's control software in order to maintain constant as-trimmed cylindrical specimen geometry while compensating for both (1) the polymer's volume expansivity coefficient $\left(7.7\left(10^{-4}\right) / \mathrm{K}\right)$ and (2) the linear contraction coefficient of $2.07 \mu \mathrm{m} / \mathrm{K}$ measured for the gap when the tooling was $8-\mathrm{mm}$ Invar plates.

Crystallization in PLA homopolymers limits the feasible temperature range for their rheological characterization to the window between the crystallization point and the onset of significant chemical (thermal, oxidative, or hydrolytic) degradation within the duration of an isothermal frequency sweep. Our PLAs, purified as described, ${ }^{8}$ were satisfactorily stable at 170 ${ }^{\circ} \mathrm{C}$ without additives, but were only marginally so at $180{ }^{\circ} \mathrm{C}$ (see Figure 1, top). Most measurements on the copolymers were made at and below $150{ }^{\circ} \mathrm{C}$ with no stabilizing additive because chemical degradation was not an issue. Only the higher molecular weights necessitated 
occasionally using data obtained at $180{ }^{\circ} \mathrm{C}$ in order to characterize the terminal relaxations. When frequency-sweep isotherms were measured at $180{ }^{\circ} \mathrm{C}$, however, exposures to that temperature were held to only a few minutes to avoid more than a few percent falloff in viscosity; dwell times at $180{ }^{\circ} \mathrm{C}$ were generally under 5 minutes, corresponding to declines of less than $5.5 \%$ in viscosity (Fig. 1, bottom). The homopolymers had tris(nonylphenyl)phosphite (TNPP) added as a stabilizer so that the measurement range could be extended to $200{ }^{\circ} \mathrm{C}$ with negligible degradation. The TNPP concentration used was $0.35 \%$, as was earlier found suitable for the vicinity of $200{ }^{\circ} \mathrm{C}$ by Lehermeier et al. ${ }^{9}$ However, instead of direct addition of neat additive to melts in an internal mixer, ${ }^{5}$ weighed quantities of the polymers were dissolved in and then recovered from fixed volumes of dichloromethane solutions containing an appropriate concentration of TNPP, by evaporation of the solvent in a vacuum oven at $40{ }^{\circ} \mathrm{C}$. This left appreciable $\mathrm{CH}_{2} \mathrm{Cl}_{2}$ dissolved in the solid polymer, but this residual solvent was removed by preheating the polymer to above the melting point (typically $\approx 180{ }^{\circ} \mathrm{C}$ ) in a vacuum oven for 5 minutes just before compression into disks ( $8 \mathrm{~mm}$ diameter $\mathrm{x} 2 \mathrm{~mm}$ thick) for rheological testing. Control samples for non-crystallizing materials showed identical flow properties as their unstabilized counterparts. 
Routine thermal characterizations of the various polymers of Table I were done using a Perkin-Elmer DSC-7 after calibration with indium. Results are collected in Table I. Glasstransition temperatures $\left(t_{\mathrm{g}}\right.$ 's) listed are half-step-height values (see Fig. 2 below). Melting points are peaks of melting endotherms in second-heating scans at $10{ }^{\circ} \mathrm{C} / \mathrm{min}$ after cooling from the melt to room temperature at the same rate. For the homopolymers, the tabulated $t_{\mathrm{g}}$ values refer to specimens quenched, as quickly as possible in the instrument, from the melts to room temperature, to suppress crystallization. $t_{\mathrm{g}}$ 's observed in samples containing crystalline phases were somewhat higher.

The use of a well-chosen phenomenological model for compact and efficient encoding and representation of complex viscosity (or complex shear modulus) is described elsewhere. ${ }^{7}$ The mathematical form of the model is tantamount to a form, named after Havriliak and Negami $(\mathrm{HN})$, that is customarily used to fit complex dielectric constant spectra. The HN model for complex viscosity is written as

$$
\eta^{*}(\omega)=\eta_{\infty}+\left(\eta_{0}-\eta_{\infty}\right)\left[1+\left(\mathrm{i} \tau_{0} \omega\right)^{\alpha}\right]^{(v-1) / \alpha}
$$

This has five easily interpretable parameters, each corresponding to a readily discernible feature in a usual plot of $\log \left|\eta^{*}\right|$ vs. $\log \omega$ : (1) $\eta_{0}$, the zero-shear viscosity, a vertical scaling factor, is the low-frequency limiting value of $\left|\eta^{*}\right|$. (2) $\tau_{0}$, a characteristic viscous relaxation time, is the horizontal scaling factor fixing the location of the transition from Newtonian to shear-thinning behavior (the "knee"). (3) $\alpha$ is an inverse measure of the breadth of the relaxation spectrum, or a direct measure of the sharpness of the knee. (4) $v-1$ is the high-frequency limiting slope $\mathrm{d}\left[\log \left|\eta^{*}(\omega)\right|\right] / \mathrm{d}(\log \omega)$ in the shear-thinning regime. (5) $\eta_{\infty}$ is the often negligible or experimentally inaccessible high-frequency limiting value of $\left|\eta^{*}\right|$ indicating onset of glassy behavior. In case $\eta_{\infty}$ is negligible, eq 1 reduces to 


$$
\eta^{*}(\omega)=\eta_{0}\left[1+\left(\mathrm{i} \tau_{0} \omega\right)^{\alpha}\right]^{(v-1) / \alpha}
$$

Taking the scalar magnitude of both sides of (1) leads to

$$
\left|\eta^{*}(\omega)\right|=\eta_{\infty}+\left(\eta_{0}-\eta_{\infty}\right)\left[1+2\left(\tau_{0} \omega\right)^{\alpha} \cos (\alpha \pi / 2)+\left(\tau_{0} \omega\right)^{2 \alpha}\right]^{\frac{\nu-1}{2 \alpha}}
$$

Similarly, from (2), for negligible $\eta_{\infty}$,

$$
\left|\eta^{*}(\omega)\right|=\eta_{0}\left[1+2\left(\tau_{0} \omega\right)^{\alpha} \cos (\alpha \pi / 2)+\left(\tau_{0} \omega\right)^{2 \alpha}\right]^{\frac{\nu-1}{2 \alpha}}
$$

Nonlinear least-squares matching of a measured $\log \left|\eta^{*}(\omega)\right|$ isothermal data set with values computed from the right-hand side of (3) or (4) produces estimates of the parameters that can be tabulated and used to conveniently compare materials and summarize their linear viscoelastic behavior.

It is of course necessary to use the form (4) when the data do not extend to sufficiently high frequencies to indicate entry into glassy behavior and hence a value for $\eta_{\infty}$. Many available data sets cover frequency ranges too low to contain adequate information about $v$, which is physically required to be a small positive constant. Graessley[Graessley, 1967 \#108; Graessley, 1974 \#21] proposed, on theoretical grounds, $v=2 / 11=0.18$. Examination of viscosities measured for model linear flexible polymers of narrowly-distributed high molecular weights and for the Curtis-Bird theoretical model indicates a smaller value of $v=0.03 \pm 0.01$; therefore $v$ is set equal to 0.03 in our least-squares fittings to determine the other parameters in eq 4.

For the noncrystallizing samples, master curves obtained by WLF shifting of data obtained over wide temperature ranges could be modeled with eq 1 modified to include a second HN mode to represent the glassy relaxation: 


$$
\eta^{*}(\omega)=\left(\eta_{0}-\eta_{\infty}\right)\left[1+\left(\mathrm{i} \tau_{0 \mathrm{t}} \omega\right)^{\alpha_{\mathrm{t}}}{ }^{\left(v_{\mathrm{t}}-1\right) / \alpha_{?}}+\eta_{\infty}\left[1+\left(\mathrm{i} \tau_{0 \mathrm{~g}} \omega\right)^{\alpha_{\mathrm{g}}}\right]^{\left(v_{\mathrm{g}}-1\right) / \alpha_{\mathrm{g}}}\right.
$$

This has separate $\tau_{0}, \alpha$, and $v$ parameters, denoted with subscripts $\mathrm{t}$ and $\mathrm{g}$, characterizing the terminal and glassy relaxations, respectively.

Master curves were constructed by least-squares matching of $\log \left|\eta^{*}(\omega)\right|$ values computed corresponding to eq 5 with measured values shifted vertically and horizontally as specified by Ferry. ${ }^{10, \text { Chapter } 11}$. Thus, shifting to a reference temperature $T_{0}$ and fitting were done all in one optimization to determine parameters of eq 5 and the constants $c_{1}{ }^{0}$ and $T_{\infty}$ (Vogel temperature) for the WLF shift factor $a_{T}$, where

$$
-\log \left(a_{T}\right)=c_{1}^{0}\left(T-T_{0}\right) /\left(T-T_{\infty}\right) .
$$

Vertical shift factors given by $\rho_{0} T_{0} / \rho T$ were applied to measured values of $G^{\prime}$ and $G^{\prime \prime}$ using the known temperature dependence of density. ${ }^{11}$ Shift factors were computed individually for every data point using the actual temperature logged from the sensor in contact with the lower rheometer plate, rather than the nominal isothermal set point.

For HN models it can be shown by numerical experiments that consistent values for plateau moduli are obtained by integrations of both relaxation spectra and loss modulus ${ }^{12}$ curves, as prescribed by Ferry ${ }^{10}$ :

$$
G_{\mathrm{N}}^{0}=\int_{\ln \tau_{\min }}^{\ln \lambda} H(\tau) \mathrm{d}(\ln \tau)=\frac{2}{\pi} \int_{-\ln \lambda}^{-\ln \tau_{\min }} G^{\prime \prime}(\omega) \mathrm{d}(\ln \omega)
$$

where, in the limits of integration, $\ln \tau_{\min }$ is the abscissa where the relaxation spectrum function $H$ has its minimum value in the transition zone and $\lambda$ is a longest relaxation time, beyond which $H(\tau)=0$ 
Plateau-modulus results can then be used to infer characteristic ratios according to the packing model. Reference [Fetters, 1999 \#473] gives the entanglement molecular weight as

$$
M_{\mathrm{e}}=\rho R T / G_{\mathrm{N}}^{0},
$$

and the same reference then gives the characteristic ratio in terms of $M_{\mathrm{e}}$ :

$$
C_{\infty}=10 \rho^{-2 / 3}\left(M_{\mathrm{e}}\right)^{-1 / 3} M_{1} / \overline{L^{2}} .
$$

Additional characteristics of chains in the melt that are available from the packing scheme are the packing length ${ }^{13}$

$$
F \frac{M}{\left\langle r^{2}\right\rangle_{0} \rho N_{\mathrm{A}}},
$$

the tube diameter

$$
d_{\mathrm{t}}=\left(\frac{M_{\mathrm{e}}\left\langle r^{2}\right\rangle_{0}}{M}\right)^{1 / 2},
$$

and the critical entanglement molecular weight ${ }^{14}$

$$
M_{\mathrm{c}}=4.24 \bar{p}^{0.65} M_{\mathrm{e}} .
$$

\section{Results and Discussion}

A typical DSC trace for a glass transition determination is shown in Figure 2. Measured glass transition temperatures from Table I are plotted in Figure 3 to show their systematic dependence on both molecular weight and comonomer proportions. The curves are fits to the data of the expected Fox-Flory form of the dependence of $T_{\mathrm{g}}$ on number-average molecular weights $\left(M_{\mathrm{n}}\right)$ :

$$
T_{\mathrm{g}}\left(M_{\mathrm{n}}\right)=A-B / M_{\mathrm{n}},
$$

where $A$ and $B$ are constants, with 


$$
A=\lim _{M_{\mathrm{n}} \rightarrow \infty} T_{\mathrm{g}}\left(M_{\mathrm{n}}\right)
$$

The $A$ values have a mild but significant dependence upon comonomer proportions, as plotted in Figure 4. Significance of the modest differences in glass transition temperatures shown in Figure 3 is confirmed by dynamic shear rheometry. This is illustrated in Figure 5, which shows well-separated maxima in $\tan \delta$ isotherms for two samples differing in $t_{\mathrm{g}}$ by $3.2^{\circ}$ as indicated by DSC.

Figure 6 shows typical master curves $\left(G^{\prime}, G^{\prime \prime}\right.$, and $\left.\left|\eta^{*}\right|\right)$ for an amorphous PLA of high molecular weight. The symbols represent measurements shifted as described above. The continuous curves represent the best-fit two-mode HN model (eq 5) with frequency shifting factors for all temperatures calculated according to eq 6 with a single pair of WLF constants. It is to be emphasized that the data is fit only to $\left|\eta^{*}\right|$ and the resulting values of $G^{\prime}$ and $G^{\text {, ' are }}$ calculated. Also, the infinite shear rate viscosity $\left(\eta_{\infty}\right)$ parameter of the terminal mode serves as the zero shear viscosity $\left(\eta_{0}\right)$ for the glassy mode. To the authors' knowledge, no other phenomenological model is capable of describing the observed viscoelastic behavior over such a large (near 14 decades) range of frequency.

The terminal mode data of Figure 6 along with similar data collected for all of the samples in Table 1 provides a means of assessing the plateau modulus and related molecular parameters for PLA. The plateau modulus value, determined using eq 7 is $1.0 \mathrm{MPa}$. Utilizing this result within the packing model provides additional insight into properties of PLA. In Table III are collected various PLA melt characteristics obtained using equations 8-12. For comparison, values determined similarly for three other typical linear flexible polymers are also tabulated. Not surprisingly, the number of backbone bonds between entanglements in PLA is somewhat 
greater than in poly(ethylene oxide) (PEO), which has the same (C-C-O-) backbone but lacks pendant oxygen and methyl groups. Correspondingly, $M_{\mathrm{c}} / M_{1}$ is also somewhat greater for PLA than for PEO. Analogous comparisons are shown between isotactic polypropylene and polyethylene, another pair having the same (C-C-) backbone but differing by pendant methyls. Notice that, as has recently been extensively discussed by Heymans, ${ }^{15}$ characteristic ratios alone do not necessarily directly indicate comparative chain stiffnesses.

\section{Conclusions}

The experimental challenges associated with obtaining high quality rheological data on PLA are non-trivial. Earlier studies on high-L content PLAs required the use of TNPP to stabilize the melts. The use of variable optical content PLAs thus holds considerable advantages in determining melt rheological properties by allowing experimetation at temperatures were degradation rates are negligible. This also provides a means for probing glassy dynamics.

Both DSC and rheometry show a small but definite effect of optical comonomer content upon the glass transition temperature, for a given average molecular weight. Glass transition temperatures increase with increasing L-content; the values of the glass transition temperatures at infinite molecular weight for 100,80 , and $50 \%$ L-content are $60.2{ }^{\circ} \mathrm{C}, 56.4{ }^{\circ} \mathrm{C}$, and $54.6{ }^{\circ} \mathrm{C}$, respectively.

The packing model used in conjunction with melt rheology measurements provides several molecular characteristics. For PLA it is found that the plateau modulus is near $1.0 \mathrm{Mpa}$ and varies in a non-systematic way with molecular weight and optical composition within a range of about $20 \%$ of this value. Corresponding molecular parameters include a packing length of 2.51 $\AA$, a tube diameter of $47.7 \AA$, and a characteristic ratio of $6.5 \pm 0.9$, independent of 
stereoisomeric composition. The critical molecular weight for entanglement, $\mathrm{M}_{\mathrm{c}}$, is found to be near $9000 \mathrm{~g} / \mathrm{mol}(9211 \mathrm{~g} / \mathrm{mol}$ for the data of Figure 6 corresponding to 383 backbone bonds). The molecular weight between entanglements, $M_{e}$, is near $4000 \mathrm{~g} / \mathrm{mol}(3959 \mathrm{~g} / \mathrm{mol}$ for the data of Figure 6 corresponding to 165 backbone bonds).

Finally, the flow properties of PLA are those of a typical linear glass forming polymer. For the weight average molecular weight range of $10^{5}$ to $10^{6}(\mathrm{~g} / \mathrm{mol})$ and a reference temperature of 180 C, the zero shear viscosity is described well by the relationship $\log \left(\eta_{0}\right)=-14.26+3.4 \log \left(M_{w}\right)$ regardless of the stereochemical composition.

\section{Acknowledgements}

The authors gratefully acknowledge funding support from the EPA and NSF under the Technologies for a Sustainable Environment program and from the U. S. Department of Energy under the Agricultural Industries of the Future program of the Office of Industrial Technologies. Discussions with Jed Randall of Cargill Dow LLC contributed to the quality of this work. 
Table I. Experimental polylactides ${ }^{\mathrm{a}}$

\begin{tabular}{|c|c|c|c|c|c|}
\hline sample & $\% \mathrm{~L} / \% \mathrm{D}$ & $10^{-3} M_{\mathrm{n}}$ & $10^{-3} M_{\mathrm{w}}$ & $t_{\mathrm{g}},{ }^{\circ} \mathrm{C}$ & $t_{\mathrm{m}},{ }^{\circ} \mathrm{C}$ \\
\hline SH-PLA-71 ${ }^{\mathrm{b}}$ & $100 / 0$ & 15.7 & 21.3 & 55.6 & 167.2 \\
\hline SH-PLA-72 ${ }^{\mathrm{b}}$ & $100 / 0$ & 77.7 & 120 & 59.5 & 177.4 \\
\hline SH-PLA-68 & $0 / 100$ & 80.0 & 124 & 58.4 & 175.9 \\
\hline SH-PLA-73 ${ }^{\mathrm{b}}$ & $100 / 0$ & 173 & 285 & 59.4 & 178.2 \\
\hline \multirow[t]{2}{*}{ SH-PLA-16 ${ }^{\mathrm{b}}$} & $100 / 0$ & 806 & 1503 & 60.3 & 177.9 \\
\hline & $100 / 0$ & $\infty$ & & 60.2 & \\
\hline SH-PLA-30 & $80 / 20$ & 3.900 & 4.56 & - & - \\
\hline SH-PLA-15 & $80 / 20$ & 14.15 & 18.3 & - & - \\
\hline SH-PLA-14 & $80 / 20$ & 70.40 & 103 & 51.5 & - \\
\hline SH-PLA-19 & $80 / 20$ & 92.50 & 142 & 56.3 & - \\
\hline SH-PLA-31 & $80 / 20$ & 351.8 & 570 & 55.2 & - \\
\hline \multirow[t]{2}{*}{ SH-PLA-13 } & $80 / 20$ & 449.8 & 692.7 & 55.7 & - \\
\hline & $80 / 20$ & $\infty$ & & 56.4 & \\
\hline SH-PLA-41 & $50 / 50$ & 14.00 & 18.3 & - & - \\
\hline SH-PLA-43 & $50 / 50$ & 84.00 & 172 & 52.0 & - \\
\hline SH-PLA-44 & $50 / 50$ & 127.0 & 245 & 51.9 & - \\
\hline \multirow[t]{2}{*}{ SH-PLA-18 } & $50 / 50$ & 288.8 & 459 & 54.1 & - \\
\hline & $50 / 50$ & $\infty$ & & 54.6 & \\
\hline $270-60-1^{\mathrm{c}}$ & $87 / 13$ & 78.46 & 152.4 & 54.7 & - \\
\hline $270-60-2^{c}$ & $94.3 / 5.7$ & 78.14 & 155.1 & 57.0 & 142.5 \\
\hline $270-60-3^{c}$ & $98.7 / 1.3$ & 71.87 & 136.0 & 59.6 & 169.0 \\
\hline $329-18-1^{\mathrm{c}}$ & $98.8 / 1.2$ & 52.54 & 96.9 & 59.3 & 172.1 \\
\hline
\end{tabular}

\footnotetext{
${ }^{a}$ Molecular weights are from SEC in THF with light-scattering detection unless noted otherwise.

${ }^{\mathrm{b}}$ Molecular weights from intrinsic viscosities in chloroform.

${ }^{\mathrm{c}}$ Molecular weights from SEC in THF using polystyrene standards and universal calibration.
} 
Table II. Typical multi-temperature isothermal frequency sweep protocol

\begin{tabular}{|c|c|c|c|}
\hline temperature, ${ }^{\circ} \mathrm{C}$ & strain amplitude, \% & actual gap, mm & commanded gap, mm \\
\hline 180 & 20 & 1.634 & 1.800 \\
\hline 150 & 20 & 1.609 & 1.734 \\
\hline 120 & 20 & 1.572 & 1.635 \\
\hline 90 & 10 & 1.536 & 1.536 \\
\hline 75 & 10 & 1.519 & 1.488 \\
\hline 65 & 0.2 & 1.507 & 1.455 \\
\hline 62 & 0.1 & 1.504 & 1.446 \\
\hline 60 & 0.1 & 1.501 & 1.439 \\
\hline 58 & 0.1 & 1.499 & 1.433 \\
\hline 56 & 0.1 & 1.497 & 1.426 \\
\hline
\end{tabular}


Table III. Flexible linear polymer melt chain dimension comparisons

\begin{tabular}{|l|c|c|c|c|}
\hline \multirow{2}{*}{ parameters } & \multicolumn{4}{|c|}{ values } \\
\cline { 2 - 5 } & PLA $\left(140{ }^{\circ} \mathrm{C}\right)$ & PEO $\left(140{ }^{\circ} \mathrm{C}\right)$ & it-PP $\left(190{ }^{\circ} \mathrm{C}\right)$ & PE $\left(140{ }^{\circ} \mathrm{C}\right)$ \\
\hline $\begin{array}{l}M_{1} \text { (relative molecular mass } \\
\text { per backbone bond) }\end{array}$ & 24.02 & 14.68 & 21.04 & 14.027 \\
\hline$\overline{L^{2}}, \AA^{2}$ & 2.05 & 2.11 & 2.37 & 2.42 \\
\hline$\rho, \mathrm{g} / \mathrm{cm}^{3}$ & 1.152 & 1.034 & 0.759 & 0.792 \\
\hline$G_{\mathrm{N}}{ }^{2}, \mathrm{MPa}$ & 1.0 & 1.8 & 0.43 & 2.6 \\
\hline$M_{\mathrm{e}}$ & 3,959 & 1,973 & 6,797 & 1,046 \\
\hline$M_{\mathrm{e}} / M_{1}$, backbone bonds & 165 & 134 & 323 & 75 \\
\hline$M_{\mathrm{c}}($ eq 20$)$ & 9,211 & 5,330 & 13,635 & 3,164 \\
\hline$M_{\mathrm{c}} / M_{1}$, backbone bonds & 383 & 363 & 648 & 226 \\
\hline$<r^{2}>_{0} / M, \AA^{2}$ & 0.574 & 0.805 & 0.694 & 1.25 \\
\hline$C_{\infty}$ (eq 17) & 6.7 & 5.6 & 6.2 & 7.3 \\
\hline$p$ (packing length), $\AA$ & 2.51 & 1.99 & 3.15 & 1.68 \\
\hline$d_{\mathrm{t}}$ (tube diameter), $\AA$ & 47.7 & 39.9 & 68.7 & 36.2 \\
\hline$d_{\mathrm{t}} / p$ (Ronca-Lin ratio) & 19.0 & 20.0 & 21.8 & 21.6 \\
\hline
\end{tabular}


Figures
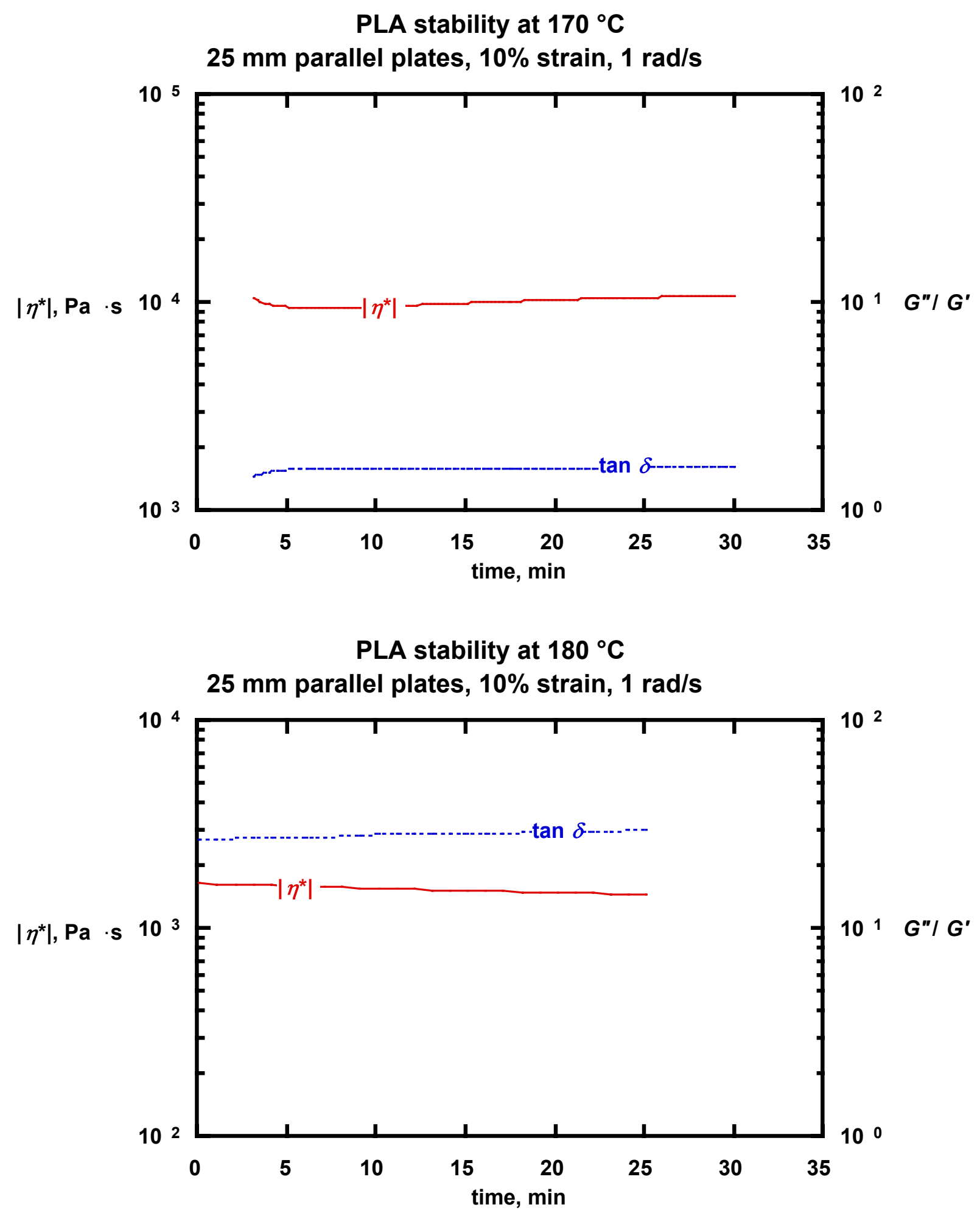

Figure 1. 


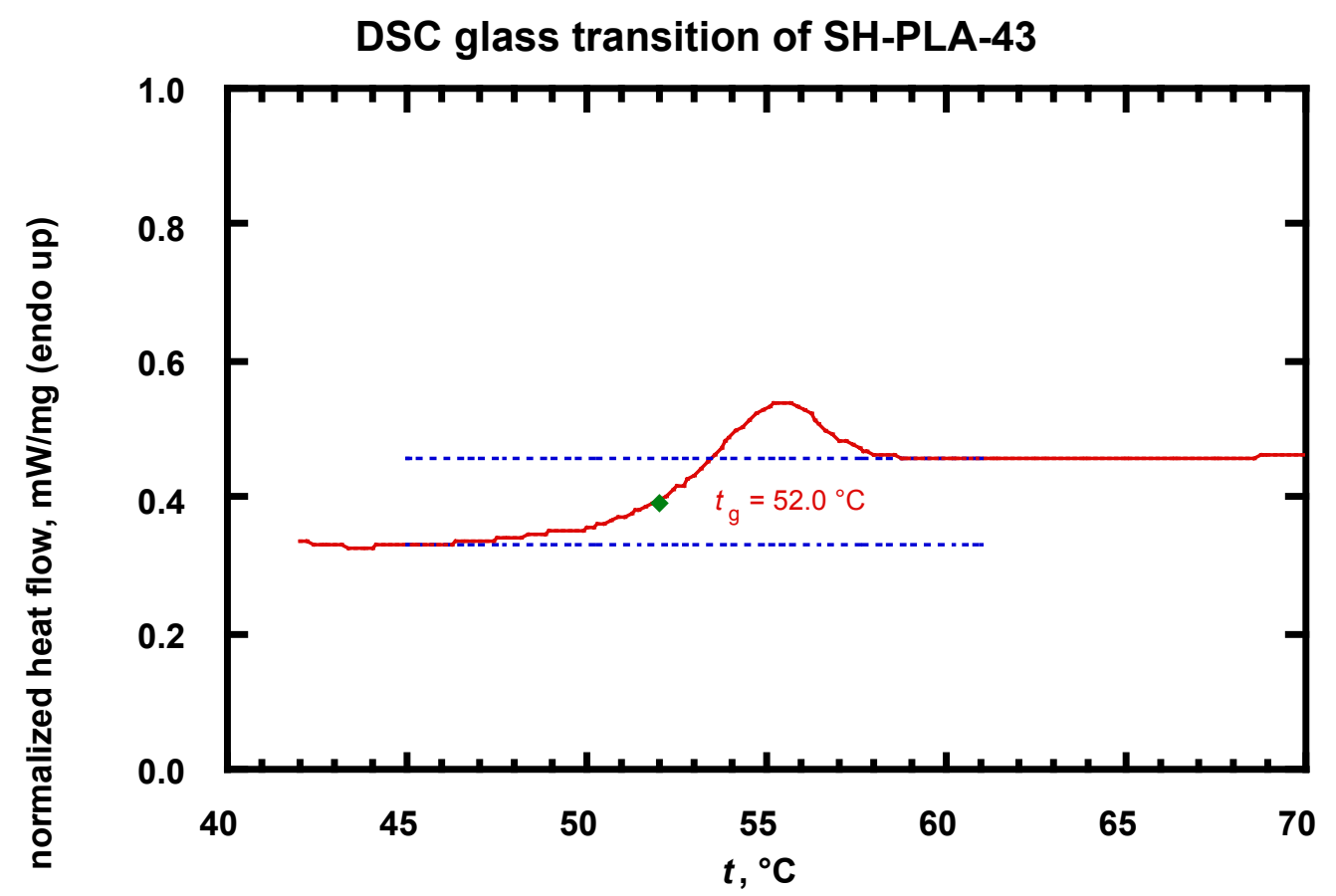

Figure 2 . 


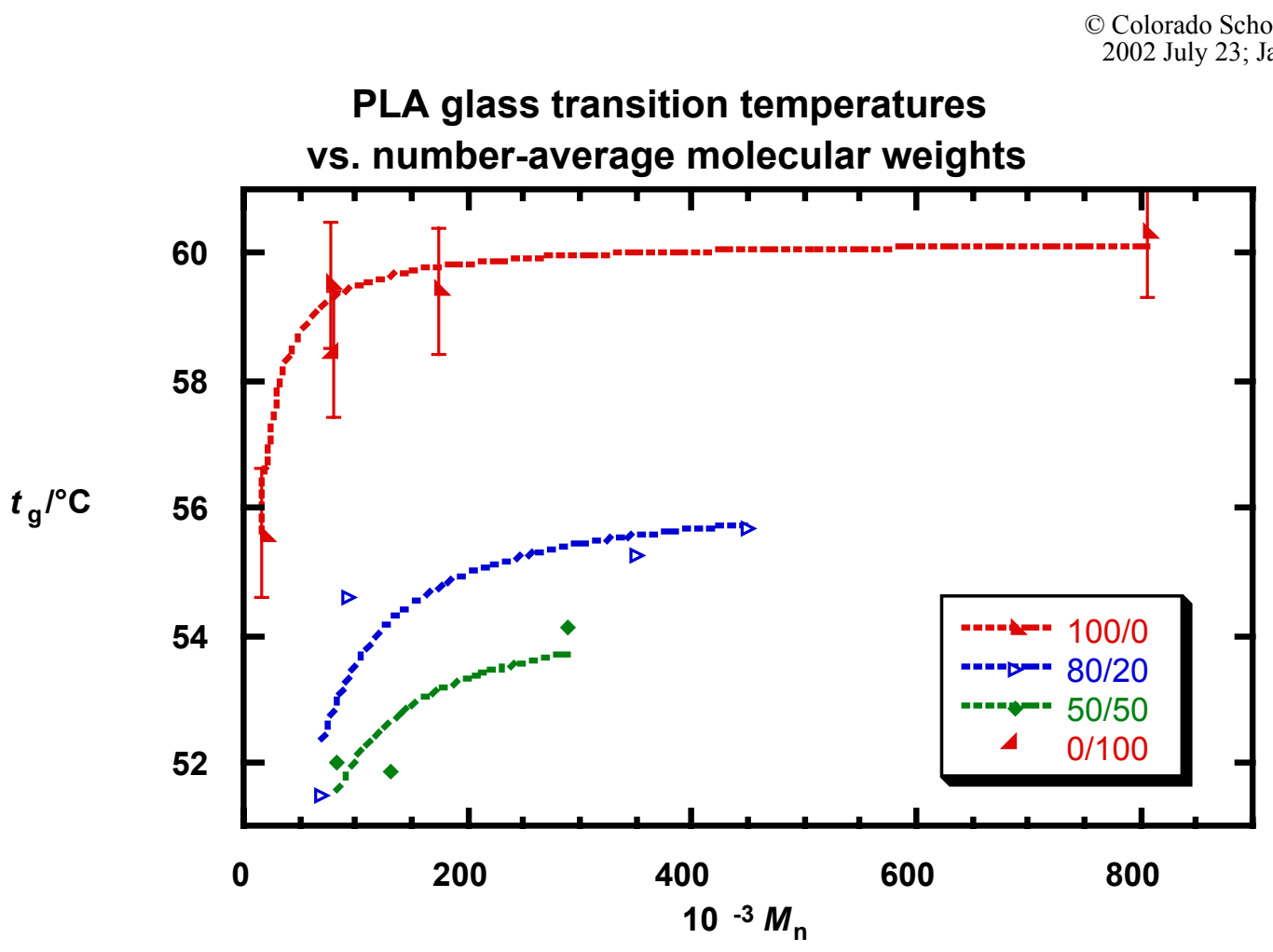

Figure 3. 


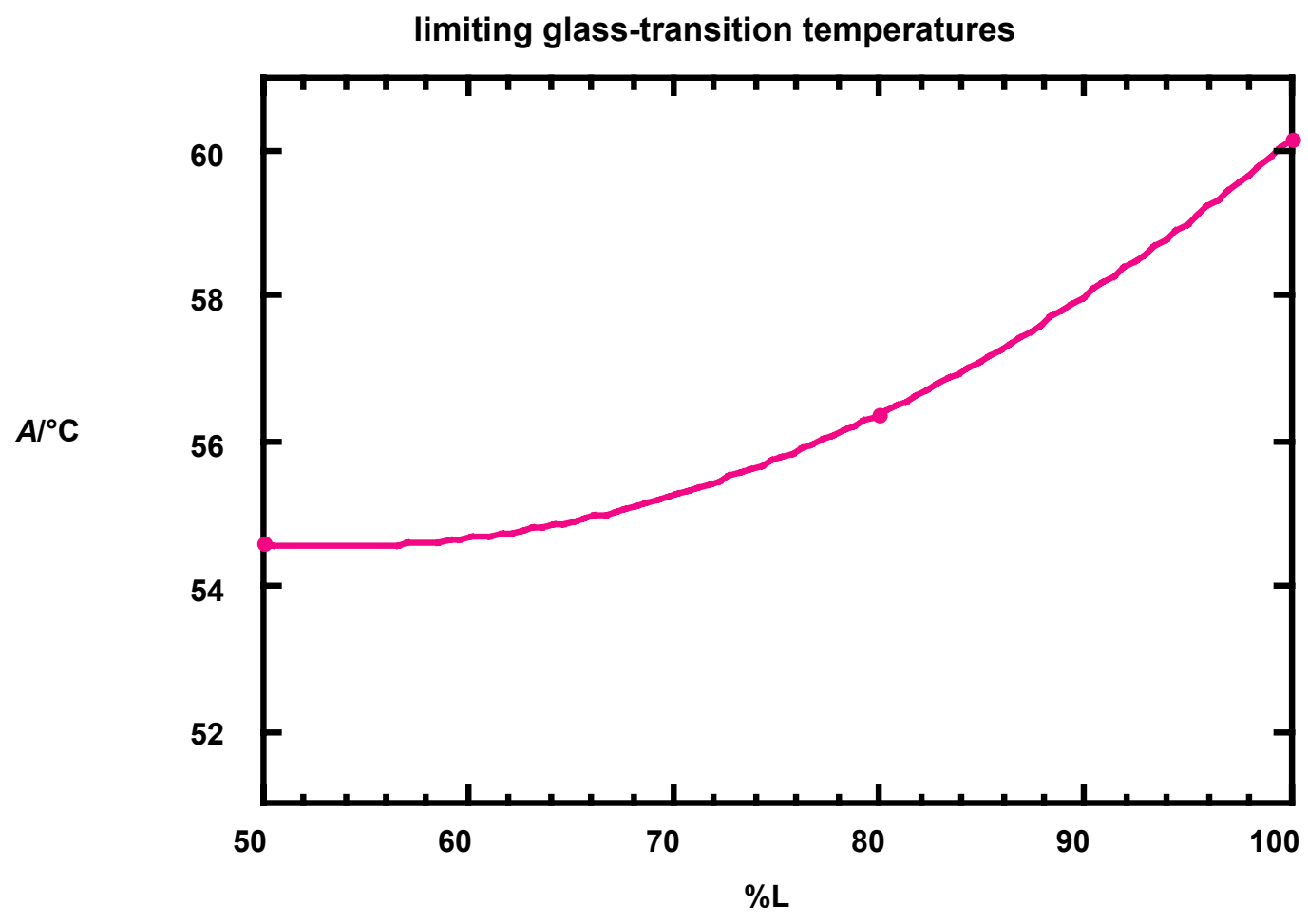

Figure 4. 


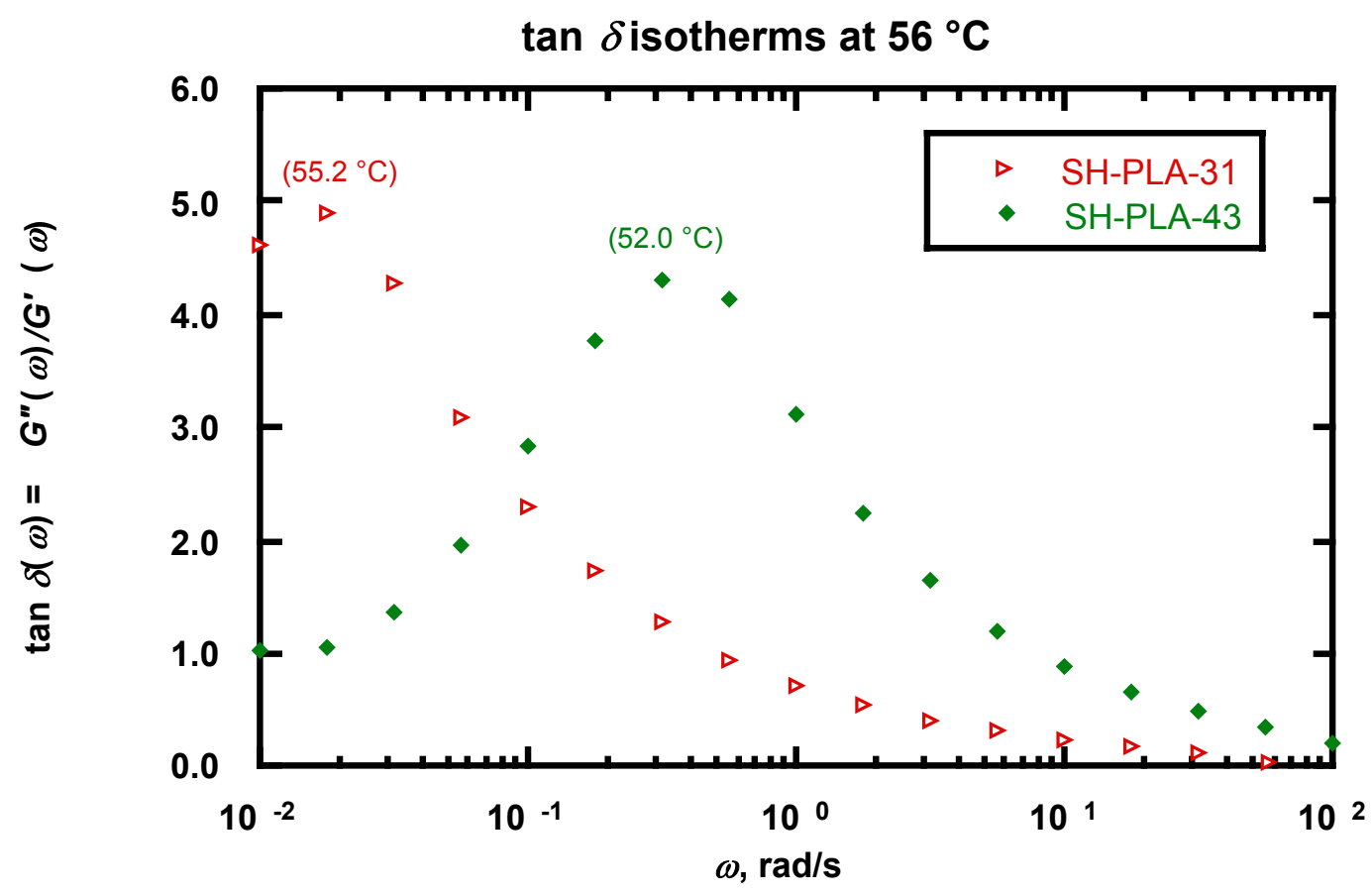

Figure 5. 
(C) 2002, Colorado School of Mines

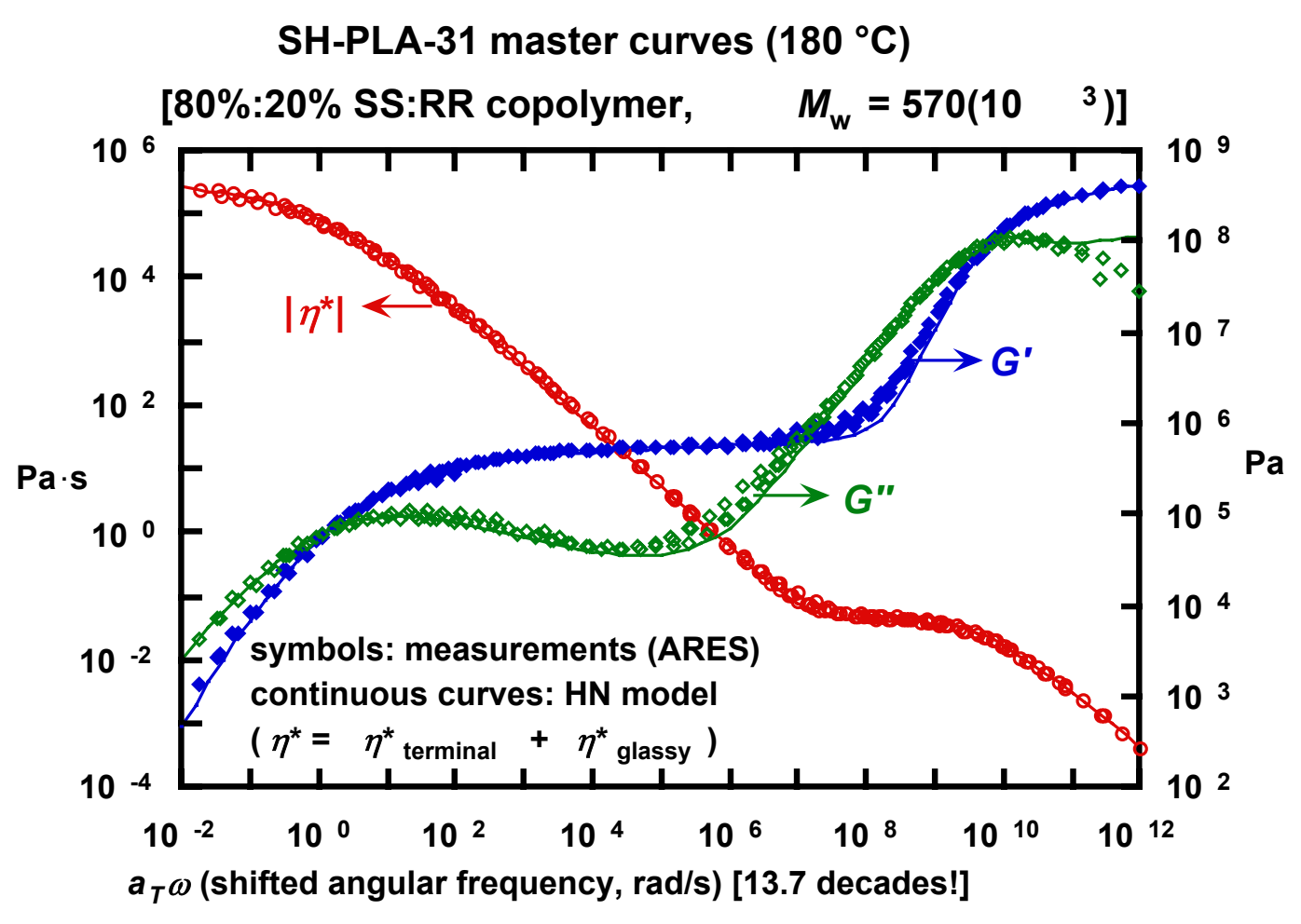

Figure 6. 
(C) 2003, Colorado School of Mines

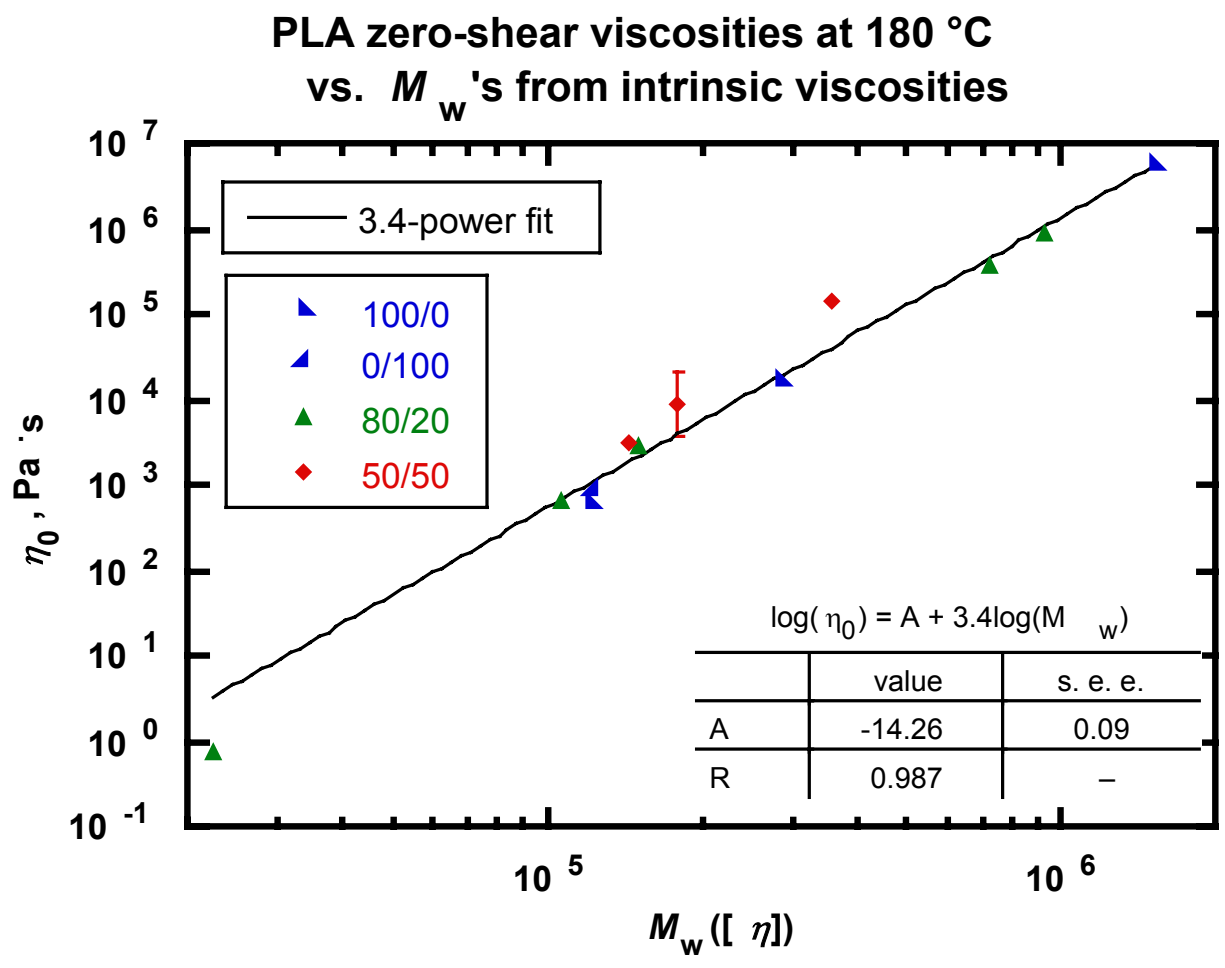

Figure 7 


\section{References:}

(1) Hartmann, M. H. In Biopolymers from Renewable Resoources; Kaplan, D. L., Ed.; Springer: Berlin, 1998; pp 367-411.

(2) Cooper-White, J. J.; Mackay, M. E. J. Polym. Sci., Part B: Polym. Phys. 1999, 37, 18031814.

(3) Dorgan, J. R.; Williams, J. S.; Lewis, D. N. J. Rheol. 1999, 43, 1141-1155.

(4) Dorgan, J. R.; Lehermeier, H.; Mang, M. J. Polym. Environ. 2000, 8, 1-9.

(5) Palade, L.-I.; Lehermeier, H. J.; Dorgan, J. R. Macromolecules 2001, 34, 1384-1390.

(6) Biresaw, G.; Carriere, C. J. J. Polym. Sci., Part B: Polym. Phys. 2002, 40, 2248-2258.

(7) Janzen, J.; Dorgan, J. R. In Annu. Tech. Conf. Soc. Plast. Eng.; Technomic: San Francisco, 2002; Vol. 46, pp 929-931.

(8) Janzen, J.; Dorgan, J. R.; Knauss, D. M.; Hait, S. B.; Limoges, B. R.; Hutchinson, M. H. Macromolecules 2003, submitted.

(9) Lehermeier, H. J.; Dorgan, J. R. Polymer and Engineering Science 2001, 41, 2172-2184.

(10) Ferry, J. D. Viscoelastic Properties of Polymers, 3rd ed.; John Wiley \& Sons: New York, 1980.

(11) Witzke, D. R. In Chemical Engineering; Michigan State University: East Lansing, Michigan, 1997; p xxvi +389.

(12) Dealy, J. M. J. Rheol. 1995, 39, 253-265.

(13) Fetters, L. J.; Lohse, D. J.; Colby, R. H. In Physical Properties of Polymers Handbook; Mark, J. E., Ed.; AIP Press: New York, 1996; pp 335-340.

(14) Fetters, L. J.; Lohse, D. J.; Milner, S. T.; Graessley, W. W. Macromolecules 1999, 32, 6847-6851.

(15) Heymans, N. Macromolecules 2000, 33, 4226-4234. 